Network Working Group

Request for Comments: 1305

Obsoletes RFC-1119, RFC-1059, RFC-958
David L. Mills

University of Delaware

March 1992

\author{
Network Time Protocol (Version 3) \\ Specification, Implementation and Analysis
}

Note: This document consists of an approximate rendering in ASCII of the Postscript document of the same name. It is provided for convenience and for use in searches, etc. However, most tables, figures, equations and captions have not been rendered and the pagination and section headings are not available.

Abstract

This document describes the Network Time Protocol (NTP), specifies its formal structure and summarizes information useful for its implementation. NTP provides the mechanisms to synchronize time and coordinate time distribution in a large, diverse internet operating at rates from mundane to lightwave. It uses a returnable-time design in which a distributed subnet of time servers operating in a selforganizing, hierarchical-master-slave configuration synchronizes local clocks within the subnet and to national time standards via wire or radio. The servers can also redistribute reference time via local routing algorithms and time daemons.

Status of this Memo

This RFC specifies an IAB standards track protocol for the Internet community and requests discussion and suggestions for improvements. Please refer to the current edition of the <169>IAB Official Protocol Standards<170> for the standardization state and status of this protocol. Distribution of this memo is unlimited.

Keywords: network clock synchronization, standard time distribution, fault-tolerant architecture, maximum-likelihood estimation, disciplined oscillator, internet protocol, high-speed networks, formal specification.

Preface

This document describes Version 3 of the Network Time Protocol (NTP). It supersedes Version 2 of the protocol described in RFC-1119 dated September 1989. However, it neither changes the protocol in any significant way nor obsoletes previous versions or existing implementations. The main motivation for the new version is to refine the analysis and implementation models for new applications at much higher network speeds to the gigabit-per-second regime and to provide for the enhanced stability, accuracy and precision required at such speeds. In particular, the sources of time and frequency errors have been rigorously examined and error bounds established in order to improve performance, provide a model for correctness assertions and indicate timekeeping quality to the user. The revision also incorporates two new optional features, (1) an algorithm to combine the offsets of a number of peer time servers in order to enhance accuracy and (2) improved local-clock algorithms which allow the poll intervals on all synchronization paths to be substantially increased in order to reduce network overhead. An overview of the changes, which are described in detail in Appendix D, follows:

1.

In Version 3 The local-clock algorithm has been overhauled to improve stability and accuracy. Appendix G presents a detailed mathematical 
model and design example which has been refined with the aid of feedback-control analysis and extensive simulation using data collected over ordinary Internet paths. Section 5 of RFC-1119 on the NTP local clock has been completely rewritten to describe the new algorithm. Since the new algorithm can result in message rates far below the old ones, it is highly recommended that they be used in new implementations. Note that use of the new algorithm does not affect interoperability with previous versions or existing implementations.

2 .

In Version 3 a new algorithm to combine the offsets of a number of peer time servers is presented in Appendix F. This algorithm is modelled on those used by national standards laboratories to combine the weighted offsets from a number of standard clocks to construct a synthetic laboratory timescale more accurate than that of any clock separately. It can be used in an NTP implementation to improve accuracy and stability and reduce errors due to asymmetric paths in the Internet. The new algorithm has been simulated using data collected over ordinary Internet paths and, along with the new local-clock algorithm, implemented and tested in the Fuzzball time servers now running in the Internet. Note that use of the new algorithm does not affect interoperability with previous versions or existing implementations.

3.

Several inconsistencies and minor errors in previous versions have been corrected in Version 3. The description of the procedures has been rewritten in pseudo-code augmented by English commentary for clarity and to avoid ambiguity. Appendix I has been added to illustrate C-language implementations of the various filtering and selection algorithms suggested for NTP. Additional information is included in Section 5 and in Appendix $E$, which includes the tutorial material formerly included in Section 2 of RFC-1119, as well as much new material clarifying the interpretation of timescales and leap seconds.

4.

Minor changes have been made in the Version-3 local-clock algorithms to avoid problems observed when leap seconds are introduced in the UTC timescale and also to support an auxiliary precision oscillator, such as a cesium clock or timing receiver, as a precision timebase. In addition, changes were made to some procedures described in section 3 and in the clock-filter and clock-selection procedures described in section 4. While these changes were made to correct minor bugs found as the result of experience and are recommended for new implementations, they do not affect interoperability with previous versions or existing implementations in other than minor ways (at least until the next leap second).

5.

In Version 3 changes were made to the way delay, offset and dispersion are defined, calculated and processed in order to reliably bound the errors inherent in the time-transfer procedures. In particular, the error accumulations were moved from the delay computation to the dispersion computation and both included in the clock filter and selection procedures. The clock-selection procedure was modified to remove the first of the two sorting/discarding steps and replace with an algorithm first proposed by Marzullo and later incorporated in the Digital Time Service. These changes do not significantly affect the ordinary operation of or compatibility with various versions of NTP, but they do provide the basis for formal statements of correctness as described in Appendix $\mathrm{H}$. Table of Contents

1. Introduction 1 


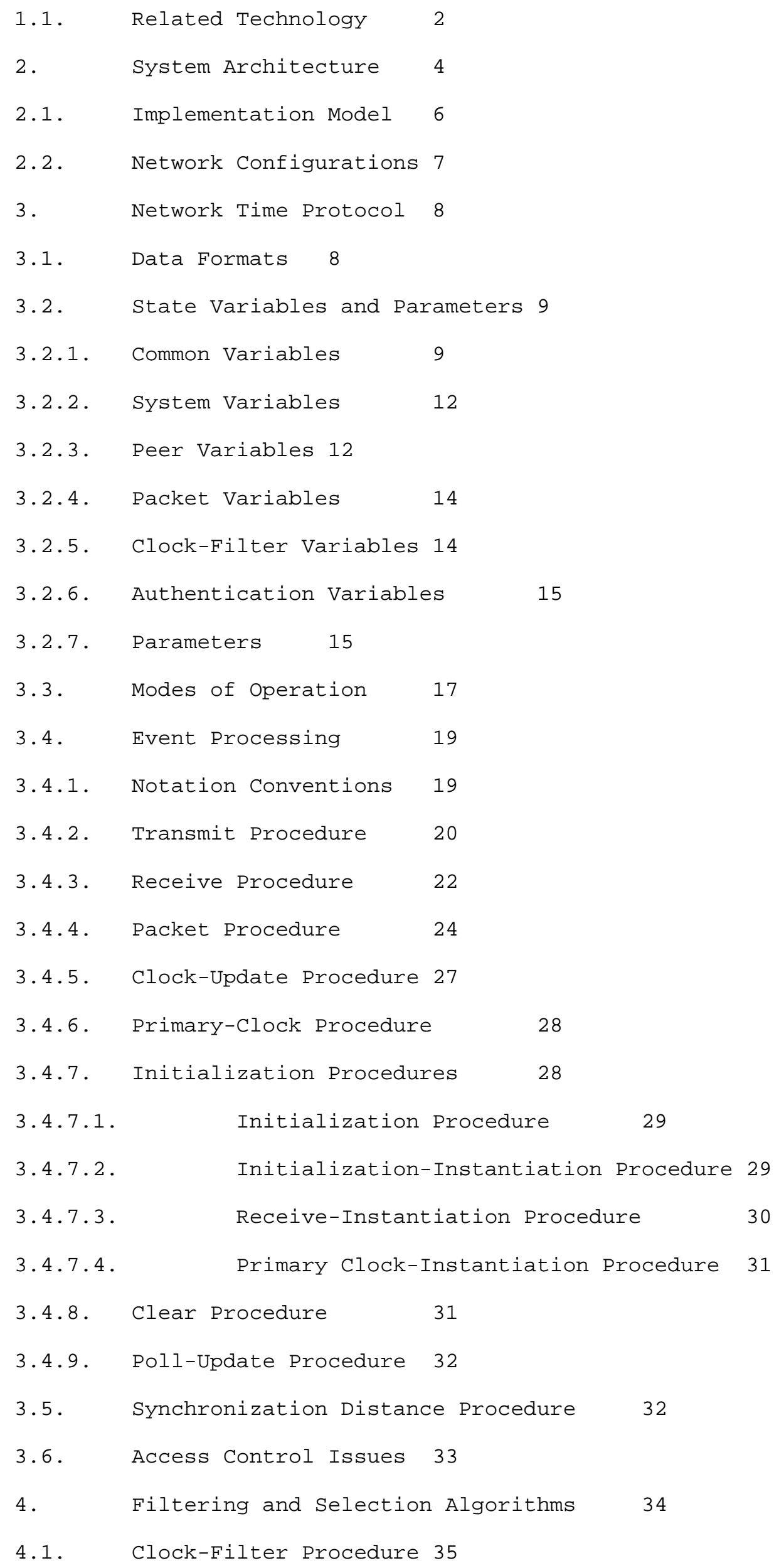




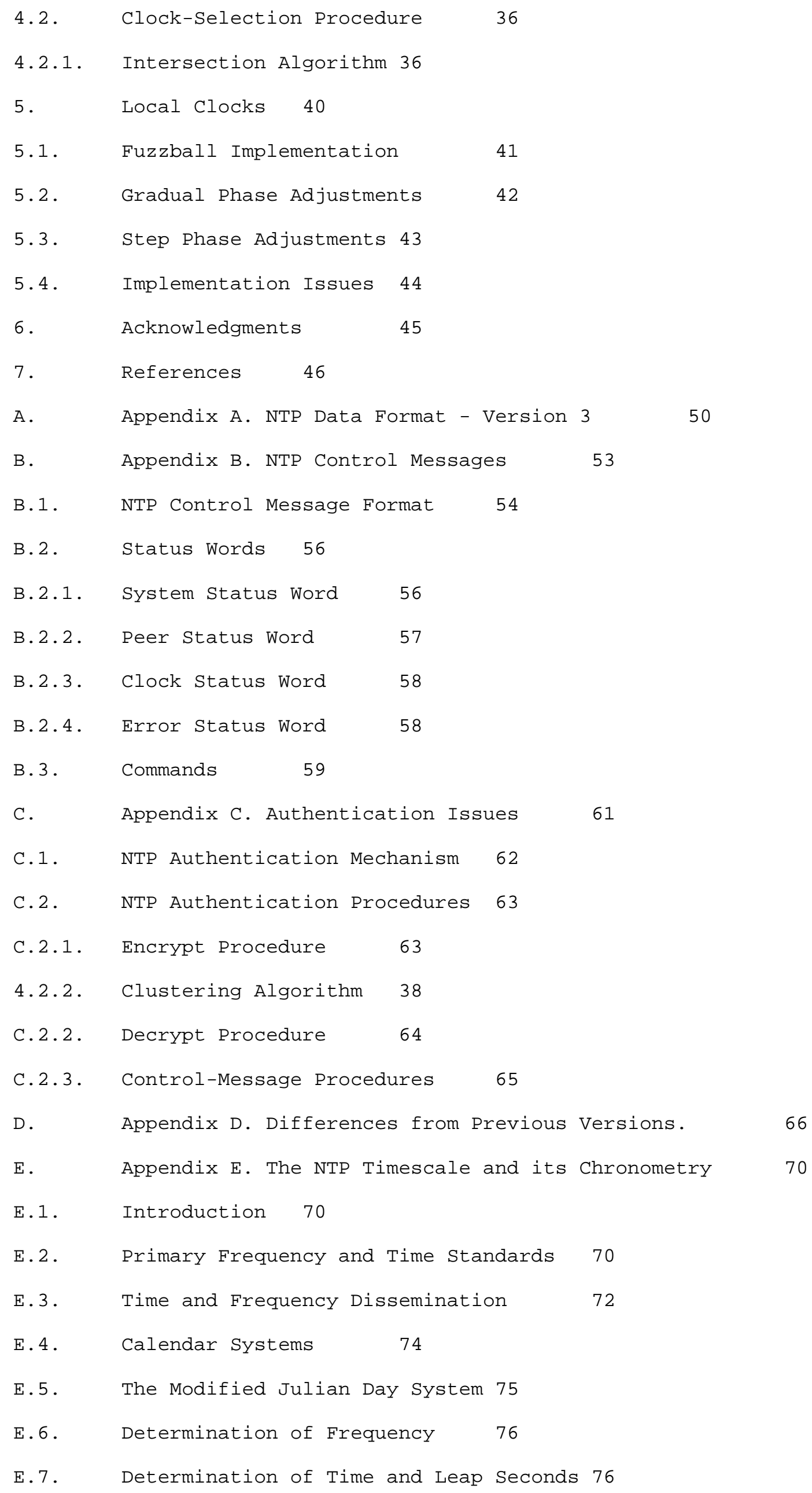


E.8. The NTP Timescale and Reckoning with UTC 78

F. Appendix F. The NTP Clock-Combining Algorithm 80

F.1. Introduction 80

F.2. Determining Time and Frequency 80

F.3. Clock Modelling 81

F.4. Development of a Composite Timescale 81

F.5. Application to NTP 84

F.6. Clock-Combining Procedure 84

G. Appendix G. Computer Clock Modelling and Analysis 86

G.1. Computer Clock Models 86

G.1.1. The Fuzzball Clock Model 88

G.1.2. The Unix Clock Model 89

G.2. Mathematical Model of the NTP Logical Clock 91

G.3. Parameter Management 93

G.4. Adjusting VCO Gain (<\$Ebold alpha $>$ ) 94

G.5. Adjusting PLL Bandwidth (<\$Ebold tau $>$ ) 94

G.6. The NTP Clock Model 95

H. Appendix H. Analysis of Errors and Correctness Principles

98

H.1. Introduction 98

H.2. Timestamp Errors 98

H.3. Measurement Errors 100

H.4. Network Errors 101

H.5. Inherited Errors $\quad 102$

H.6. Correctness Principles 104

I. Appendix I. Selected C-Language Program Listings 107

I.1. Common Definitions and Variables 107

I.2. Clock<196>Filter Algorithm 108

I.3. Interval Intersection Algorithm 109

I.4. Clock<196>Selection Algorithm 110

I.5. Clock<196>Combining Procedure 111

I.6. Subroutine to Compute Synchronization Distance 112

List of Figures 
Figure 1. Implementation Model 6

Figure 2. Calculating Delay and Offset 25

Figure 3. Clock Registers 39

Figure 4. NTP Message Header 50

Figure 5. NTP Control Message Header 54

Figure 6. Status Word Formats 55

Figure 7. Authenticator Format 63

Figure 8. Comparison of UTC and NTP Timescales at Leap 79

Figure 9. Network Time Protocol 80

Figure 10. Hardware Clock Models 86

Figure 11. Clock Adjustment Process 90

Figure 12. NTP Phase-Lock Loop (PLL) Model 91

Figure 13. Timing Intervals 96

Figure 14. Measuring Delay and Offset 100

Figure 15. Error Accumulations 103

Figure 16. Confidence Intervals and Intersections

List of Tables

Table 1. System Variables

Table 2. Peer Variables 13

Table 3. Packet Variables

Table 4. Parameters 16

Table 5. Modes and Actions 22

Table 6. Clock Parameters 40

Table 7. Characteristics of Standard Oscillators

Table 8. Table of Leap-Second Insertions

77

Table 9. Notation Used in PLL Analysis 91

Table 10. PLL Parameters

91

Table 11. Notation Used in PLL Analysis 95

Table 12. Notation Used in Error Analysis

Introduction

This document constitutes a formal specification of the Network Time Protocol (NTP) Version 3, which is used to synchronize timekeeping among a set of distributed time servers and clients. It defines the architectures, algorithms, entities and protocols used by NTP and is intended primarily for implementors. A companion document [MIL91a] summarizes the requirements, analytical models, algorithmic analysis and performance under typical Internet conditions. Another document [MIL91b] describes the NTP timescale and its relationship to other standard 
timescales now in use. NTP was first described in RFC-958 [MIL85c], but has since evolved in significant ways, culminating in the most recent NTP Version 2 described in RFC-1119 [MIL89]. It is built on the Internet Protocol (IP) [DAR81a] and User Datagram Protocol (UDP) [POS80], which provide a connectionless transport mechanism; however, it is readily adaptable to other protocol suites. NTP is evolved from the Time Protocol [POS83b] and the ICMP Timestamp message [DAR81b], but is specifically designed to maintain accuracy and robustness, even when used over typical Internet paths involving multiple gateways, highly dispersive delays and unreliable nets.

The service environment consists of the implementation model and service model described in section 2. The implementation model is based on a multiple-process operating system architecture, although other architectures could be used as well. The service model is based on a returnable-time design which depends only on measured clock offsets, but does not require reliable message delivery. The synchronization subnet uses a self-organizing, hierarchical-master-slave configuration, with synchronization paths determined by a minimum-weight spanning tree. While multiple masters (primary servers) may exist, there is no requirement for an election protocol.

NTP itself is described in Section 3. It provides the protocol mechanisms to synchronize time in principle to precisions in the order of nanoseconds while preserving a non-ambiguous date well into the next century. The protocol includes provisions to specify the characteristics and estimate the error of the local clock and the time server to which it may be synchronized. It also includes provisions for operation with a number of mutually suspicious, hierarchically distributed primary reference sources such as radio-synchronized clocks.

Section 4 describes algorithms useful for deglitching and smoothing clock-offset samples collected on a continuous basis. These algorithms evolved from those suggested in [MIL85a], were refined as the results of experiments described in [MIL85b] and further evolved under typical operating conditions over the last three years. In addition, as the result of experience in operating multiple-server subnets including radio clocks at several sites in the U.S. and with clients in the U.S. and Europe, reliable algorithms for selecting good clocks from a population possibly including broken ones have been developed [DEC89], [MIL91a] and are described in section 4.

The accuracies achievable by NTP depend strongly on the precision of the local-clock hardware and stringent control of device and process latencies. Provisions must be included to adjust the software logicalclock time and frequency in response to corrections produced by NTP. Section 5 describes a local-clock design evolved from the Fuzzball implementation described in [MIL83b] and [MIL88b]. This design includes offset-slewing, frequency compensation and deglitching mechanisms capable of accuracies in the order of a millisecond, even after extended periods when synchronization to primary reference sources has been lost.

Details specific to NTP packet formats used with the Internet Protocol (IP) and User Datagram Protocol (UDP) are presented in Appendix A, while details of a suggested auxiliary NTP Control Message, which may be used when comprehensive network-monitoring facilities are not available, are presented in Appendix B. Appendix C contains specification and implementation details of an optional authentication mechanism which can be used to control access and prevent unauthorized data modification, while Appendix D contains a listing of differences between Version 3 of NTP and previous versions. Appendix E expands on issues involved with precision timescales and calendar dating peculiar to computer networks and NTP. Appendix F describes an optional algorithm to improve accuracy by combining the time offsets of a number of clocks. Appendix G presents a detailed mathematical model and analysis of the NTP local-clock algorithms. Appendix $\mathrm{H}$ analyzes the sources and propagation of errors and presents correctness principles relating to the time-transfer 
service. Appendix I illustrates C-language code segments for the clockfilter, clock-selection and related algorithms described in section 4.

Related Technology

Other mechanisms have been specified in the Internet protocol suite to record and transmit the time at which an event takes place, including the Daytime protocol [POS83a], Time Protocol [Pos83b], ICMP Timestamp message [DAR81b] and IP Timestamp option [SU81]. Experimental results on measured clock offsets and roundtrip delays in the Internet are discussed in [MIL83a], [MIL85b], [COL88] and [MIL88a]. Other synchronization algorithms are discussed in [LAM78], [GUS84], [HAL84], [LUN84], [LAM85], [MAR85], [MIL85a], [MIL85b], [MIL85c], [GUS85b], $[\mathrm{SCH} 86],[\mathrm{TRI} 86],[\mathrm{RIC88}]$, [MIL88a], [DEC89] and [MIL91a], while protocols based on them are described in [MIL81a], [MIL81b], [MIL83b], [GUS85a], [MIL85c], [TRI86], [MIL88a], [DEC89] and [MIL91a]. NTP uses techniques evolved from them and both linear-systems and agreement methodologies. Linear methods for digital telephone network synchronization are summarized in [LIN80], while agreement methods for clock synchronization are summarized in [LAM85].

The Digital Time Service (DTS) [DEC89] has many of the same service objectives as NTP. The DTS design places heavy emphasis on configuration management and correctness principles when operated in a managed LAN or LAN-cluster environment, while NTP places heavy emphasis on the accuracy and stability of the service operated in an unmanaged, global-internet environment. In DTS a synchronization subnet consists of clerks, servers, couriers and time providers. With respect to the NTP nomenclature, a time provider is a primary reference source, a courier is a secondary server intended to import time from one or more distant primary servers for local redistribution and a server is intended to provide time for possibly many end nodes or clerks. Unlike NTP, DTS does not need or use mode or stratum information in clock selection and does not include provisions to filter timing noise, select the most accurate from a set of presumed correct clocks or compensate for inherent frequency errors.

In fact, the latest revisions in NTP have adopted certain features of DTS in order to support correctness principles. These include mechanisms to bound the maximum errors inherent in the time-transfer procedures and the use of a provably correct (subject to stated assumptions) mechanism to reject inappropriate peers in the clock-selection procedures. These features are described in Section 4 and Appendix $H$ of this document.

The Fuzzball routing protocol [MIL83b], sometimes called Hellospeak, incorporates time synchronization directly into the routing-protocol design. One or more processes synchronize to an external reference source, such as a radio clock or NTP daemon, and the routing algorithm constructs a minimum-weight spanning tree rooted on these processes. The clock offsets are then distributed along the arcs of the spanning tree to all processes in the system and the various process clocks corrected using the procedure described in section 5 of this document. While it can be seen that the design of Hellospeak strongly influenced the design of NTP, Hellospeak itself is not an Internet protocol and is unsuited for use outside its local-net environment.

The Unix 4.3bsd time daemon timed [GUS85a] uses a single master-time daemon to measure offsets of a number of slave hosts and send periodic corrections to them. In this model the master is determined using an election algorithm [GUS85b] designed to avoid situations where either no master is elected or more than one master is elected. The election process requires a broadcast capability, which is not a ubiquitous feature of the Internet. While this model has been extended to support hierarchical configurations in which a slave on one network serves as a master on the other [TRI86], the model requires handcrafted configuration tables in order to establish the hierarchy and avoid loops. In addition to the burdensome, but presumably infrequent, 
overheads of the election process, the offset measurement/correction process requires twice as many messages as NTP per update.

A scheme with features similar to NTP is described in [KOP87]. This scheme is intended for multi-server LANs where each of a set of possibly many time servers determines its local-time offset relative to each of the other servers in the set using periodic timestamped messages, then determines the local-clock correction using the Fault-Tolerant Average (FTA) algorithm of [LUN84]. The FTA algorithm, which is useful where up to $\mathrm{k}$ servers may be faulty, sorts the offsets, discards the $\mathrm{k}$ highest and lowest ones and averages the rest. The scheme, as described in [SCH86], is most suitable to LAN environments which support broadcast and would result in unacceptable overhead in an internet environment. In addition, for reasons given in section 4 of this paper, the statistical properties of the FTA algorithm are not likely to be optimal in an internet environment with highly dispersive delays.

A good deal of research has gone into the issue of maintaining accurate time in a community where some clocks cannot be trusted. A truechimer is a clock that maintains timekeeping accuracy to a previously published (and trusted) standard, while a falseticker is a clock that does not. Determining whether a particular clock is a truechimer or falseticker is an interesting abstract problem which can be attacked using agreement methods summarized in [LAM85] and [SRI87].

A convergence function operates upon the offsets between the clocks in a system to increase the accuracy by reducing or eliminating errors caused by falsetickers. There are two classes of convergence functions, those involving interactive-convergence algorithms and those involving interactive-consistency algorithms. Interactive-convergence algorithms use statistical clustering techniques such as the fault-tolerant average algorithm of [HAL84], the CNV algorithm of [LUN84], the majority-subset algorithm of [MIL85a], the non-Byzantine algorithm of [RIC88], the egocentric algorithm of [SCH86], the intersection algorithm of [MAR85] and [DEC89] and the algorithms in section 4 of this document.

Interactive-consistency algorithms are designed to detect faulty clock processes which might indicate grossly inconsistent offsets in successive readings or to different readers. These algorithms use an agreement protocol involving successive rounds of readings, possibly relayed and possibly augmented by digital signatures. Examples include the fireworks algorithm of [HAL84] and the optimum algorithm of [SRI87]. However, these algorithms require large numbers of messages, especially when large numbers of clocks are involved, and are designed to detect faults that have rarely been found in the Internet experience. For these reasons they are not considered further in this document.

In practice it is not possible to determine the truechimers from the falsetickers on other than a statistical basis, especially with hierarchical configurations and a statistically noisy Internet. While it is possible to bound the maximum errors in the time-transfer procedures, assuming sufficiently generous tolerances are adopted for the hardware components, this generally results in rather poor accuracies and stabilities. The approach taken in the NTP design and its predecessors involves mutually coupled oscillators and maximum-likelihood estimation and clock-selection procedures, together with a design that allows provable assertions on error bounds to be made relative to stated assumptions on the correctness of the primary reference sources. From the analytical point of view, the system of distributed NTP peers operates as a set of coupled phase-locked oscillators, with the update algorithm functioning as a phase detector and the local clock as a disciplined oscillator, but with deterministic error bounds calculated at each step in the time-transfer process.

The particular choice of offset measurement and computation procedure described in section 3 is a variant of the returnable-time system used in some digital telephone networks [LIN80]. The clock filter and 
selection algorithms are designed so that the clock synchronization subnet self-organizes into a hierarchical-master-slave configuration [MIT80]. With respect to timekeeping accuracy and stability, the similarity of NTP to digital telephone systems is not accidental, since systems like this have been studied extensively [LIN80], [BRA80]. What makes the NTP model unique is the adaptive configuration, polling, filtering, selection and correctness mechanisms which tailor the dynamics of the system to fit the ubiquitous Internet environment.

System Architecture

In the NTP model a number of primary reference sources, synchronized by wire or radio to national standards, are connected to widely accessible resources, such as backbone gateways, and operated as primary time servers. The purpose of NTP is to convey timekeeping information from these servers to other time servers via the Internet and also to crosscheck clocks and mitigate errors due to equipment or propagation failures. Some number of local-net hosts or gateways, acting as secondary time servers, run NTP with one or more of the primary servers. In order to reduce the protocol overhead, the secondary servers distribute time via NTP to the remaining local-net hosts. In the interest of reliability, selected hosts can be equipped with less accurate but less expensive radio clocks and used for backup in case of failure of the primary and/or secondary servers or communication paths between them.

Throughout this document a standard nomenclature has been adopted: the stability of a clock is how well it can maintain a constant frequency, the accuracy is how well its frequency and time compare with national standards and the precision is how precisely these quantities can be maintained within a particular timekeeping system. Unless indicated otherwise, the offset of two clocks is the time difference between them, while the skew is the frequency difference (first derivative of offset with time) between them. Real clocks exhibit some variation in skew (second derivative of offset with time), which is called drift; however, in this version of the specification the drift is assumed zero.

NTP is designed to produce three products: clock offset, roundtrip delay and dispersion, all of which are relative to a selected reference clock. clock offset represents the amount to adjust the local clock to bring it into correspondence with the reference clock. Roundtrip delay provides the capability to launch a message to arrive at the reference clock at a specified time. Dispersion represents the maximum error of the local clock relative to the reference clock. Since most host time servers will synchronize via another peer time server, there are two components in each of these three products, those determined by the peer relative to the primary reference source of standard time and those measured by the host relative to the peer. Each of these components are maintained separately in the protocol in order to facilitate error control and management of the subnet itself. They provide not only precision measurements of offset and delay, but also definitive maximum error bounds, so that the user interface can determine not only the time, but the quality of the time as well.

There is no provision for peer discovery or virtual-circuit management in NTP. Data integrity is provided by the IP and UDP checksums. No flowcontrol or retransmission facilities are provided or necessary. Duplicate detection is inherent in the processing algorithms. The service can operate in a symmetric mode, in which servers and clients are indistinguishable, yet maintain a small amount of state information, or in client/server mode, in which servers need maintain no state other than that contained in the client request. A lightweight associationmanagement capability, including dynamic reachability and variable pollrate mechanisms, is included only to manage the state information and reduce resource requirements. Since only a single NTP message format is used, the protocol is easily implemented and can be used in a variety of solicited or unsolicited polling mechanisms. 
It should be recognized that clock synchronization requires by its nature long periods and multiple comparisons in order to maintain accurate timekeeping. While only a few measurements are usually adequate to reliably determine local time to within a second or so, periods of many hours and dozens of measurements are required to resolve oscillator skew and maintain local time to the order of a millisecond. Thus, the accuracy achieved is directly dependent on the time taken to achieve it. Fortunately, the frequency of measurements can be quite low and almost always non-intrusive to normal net operations.

Implementation Model

In what may be the most common client/server model a client sends an NTP message to one or more servers and processes the replies as received. The server interchanges addresses and ports, overwrites certain fields in the message, recalculates the checksum and returns the message immediately. Information included in the NTP message allows the client to determine the server time with respect to local time and adjust the local clock accordingly. In addition, the message includes information to calculate the expected timekeeping accuracy and reliability, as well as select the best from possibly several servers.

While the client/server model may suffice for use on local nets involving a public server and perhaps many workstation clients, the full generality of NTP requires distributed participation of a number of client/servers or peers arranged in a dynamically reconfigurable, hierarchically distributed configuration. It also requires sophisticated algorithms for association management, data manipulation and local-clock control. Throughout the remainder of this document the term host refers to an instantiation of the protocol on a local processor, while the term peer refers to the instantiation of the protocol on a remote processor connected by a network path.

Figure 1<\$\&figl> shows an implementation model for a host including three processes sharing a partitioned data base, with a partition dedicated to each peer, and interconnected by a message-passing system. The transmit process, driven by independent timers for each peer, collects information in the data base and sends NTP messages to the peers. Each message contains the local timestamp when the message is sent, together with previously received timestamps and other information necessary to determine the hierarchy and manage the association. The message transmission rate is determined by the accuracy required of the local clock, as well as the accuracies of its peers.

The receive process receives NTP messages and perhaps messages in other protocols, as well as information from directly connected radio clocks. When an NTP message is received, the offset between the peer clock and the local clock is computed and incorporated into the data base along with other information useful for error determination and peer selection. A filtering algorithm described in section 4 improves the accuracy by discarding inferior data.

The update procedure is initiated upon receipt of a message and at other times. It processes the offset data from each peer and selects the best one using the algorithms of Section 4. This may involve many observations of a few peers or a few observations of many peers, depending on the accuracies required.

The local-clock process operates upon the offset data produced by the update procedure and adjusts the phase and frequency of the local clock using the mechanisms described in section 5. This may result in either a step-change or a gradual phase adjustment of the local clock to reduce the offset to zero. The local clock provides a stable source of time information to other users of the system and for subsequent reference by NTP itself. 
The synchronization subnet is a connected network of primary and secondary time servers, clients and interconnecting transmission paths. A primary time server is directly synchronized to a primary reference source, usually a radio clock. A secondary time server derives synchronization, possibly via other secondary servers, from a primary server over network paths possibly shared with other services. Under normal circumstances it is intended that the synchronization subnet of primary and secondary servers assumes a hierarchical-master-slave configuration with the primary servers at the root and secondary servers of decreasing accuracy at successive levels toward the leaves.

Following conventions established by the telephone industry [BEL86], the accuracy of each server is defined by a number called the stratum, with the topmost level (primary servers) assigned as one and each level downwards (secondary servers) in the hierarchy assigned as one greater than the preceding level. With current technology and available radio clocks, single-sample accuracies in the order of a millisecond can be achieved at the network interface of a primary server. Accuracies of this order require special care in the design and implementation of the operating system and the local-clock mechanism, such as described in Section 5 .

As the stratum increases from one, the single-sample accuracies achievable will degrade depending on the network paths and local-clock stabilities. In order to avoid the tedious calculations [BRA80] necessary to estimate errors in each specific configuration, it is useful to assume the mean measurement errors accumulate approximately in proportion to the measured delay and dispersion relative to the root of the synchronization subnet. Appendix H contains an analysis of errors, including a derivation of maximum error as a function of delay and dispersion, where the latter quantity depends on the precision of the timekeeping system, frequency tolerance of the local clock and various residuals. Assuming the primary servers are synchronized to standard time within known accuracies, this provides a reliable, determistic specification on timekeeping accuracies throughout the synchronization subnet.

Again drawing from the experience of the telephone industry, which learned such lessons at considerable cost [ABA89], the synchronization subnet topology should be organized to produce the highest accuracy, but must never be allowed to form a loop. An additional factor is that each increment in stratum involves a potentially unreliable time server which introduces additional measurement errors. The selection algorithm used in NTP uses a variant of the Bellman-Ford distributed routing algorithm [37] to compute the minimum-weight spanning trees rooted on the primary servers. The distance metric used by the algorithm consists of the (scaled) stratum plus the synchronization distance, which itself consists of the dispersion plus one-half the absolute delay. Thus, the synchronization path will always take the minimum number of servers to the root, with ties resolved on the basis of maximum error.

As a result of this design, the subnet reconfigures automatically in a hierarchical-master-slave configuration to produce the most accurate and reliable time, even when one or more primary or secondary servers or the network paths between them fail. This includes the case where all normal primary servers (e.g., highly accurate WWVB radio clock operating at the lowest synchronization distances) on a possibly partitioned subnet fail, but one or more backup primary servers (e.g., less accurate WWV radio clock operating at higher synchronization distances) continue operation. However, should all primary servers throughout the subnet fail, the remaining secondary servers will synchronize among themselves while distances ratchet upwards to a preselected maximum <169>infinity<170> due to the well-known properties of the Bellman-Ford algorithm. Upon reaching the maximum on all paths, a server will drop off the subnet and free-run using its last determined time and frequency. Since these 
computations are expected to be very precise, especially in frequency, even extended outage periods can result in timekeeping errors not greater than a few milliseconds per day with appropriately stabilized oscillators (see section 5).

In the case of multiple primary servers, the spanning-tree computation will usually select the server at minimum synchronization distance. However, when these servers are at approximately the same distance, the computation may result in random selections among them as the result of normal dispersive delays. Ordinarily, this does not degrade accuracy as long as any discrepancy between the primary servers is small compared to the synchronization distance. If not, the filter and selection algorithms will select the best of the available servers and cast out outlyers as intended.

\section{Network Time Protocol}

This section consists of a formal definition of the Network Time Protocol, including its data formats, entities, state variables, events and event-processing procedures. The specification is based on the implementation model illustrated in Figure 1, but it is not intended that this model is the only one upon which a specification can be based. In particular, the specification is intended to illustrate and clarify the intrinsic operations of NTP, as well as to serve as a foundation for a more rigorous, comprehensive and verifiable specification.

\section{Data Formats}

All mathematical operations expressed or implied herein are in two'scomplement, fixed-point arithmetic. Data are specified as integer or fixed-point quantities, with bits numbered in big-endian fashion from zero starting at the left, or high-order, position. Since various implementations may scale externally derived quantities for internal use, neither the precision nor decimal-point placement for fixed-point quantities is specified. Unless specified otherwise, all quantities are unsigned and may occupy the full field width with an implied zero preceding bit zero. Hardware and software packages designed to work with signed quantities will thus yield surprising results when the most significant (sign) bit is set. It is suggested that externally derived, unsigned fixed-point quantities such as timestamps be shifted right one bit for internal use, since the precision represented by the full field width is seldom justified.

Since NTP timestamps are cherished data and, in fact, represent the main product of the protocol, a special timestamp format has been established. NTP timestamps are represented as a 64-bit unsigned fixedpoint number, in seconds relative to oh on 1 January 1900 . The integer part is in the first 32 bits and the fraction part in the last 32 bits. This format allows convenient multiple-precision arithmetic and conversion to Time Protocol representation (seconds), but does complicate the conversion to ICMP Timestamp message representation (milliseconds). The precision of this representation is about 200 picoseconds, which should be adequate for even the most exotic requirements.

Timestamps are determined by copying the current value of the local clock to a timestamp when some significant event, such as the arrival of a message, occurs. In order to maintain the highest accuracy, it is important that this be done as close to the hardware or software driver associated with the event as possible. In particular, departure timestamps should be redetermined for each link-level retransmission. In some cases a particular timestamp may not be available, such as when the host is rebooted or the protocol first starts up. In these cases the 64bit field is set to zero, indicating the value is invalid or undefined.

Note that since some time in 1968 the most significant bit (bit 0 of the integer part) has been set and that the 64-bit field will overflow some 
time in 2036. Should NTP be in use in 2036, some external means will be necessary to qualify time relative to 1900 and time relative to 2036 (and other multiples of 136 years). Timestamped data requiring such qualification will be so precious that appropriate means should be readily available. There will exist an 200-picosecond interval, henceforth ignored, every 136 years when the 64-bit field will be zero and thus considered invalid.

State Variables and Parameters

Following is a summary of the various state variables and parameters used by the protocol. They are separated into classes of system variables, which relate to the operating system environment and localclock mechanism; peer variables, which represent the state of the protocol machine specific to each peer; packet variables, which represent the contents of the NTP message; and parameters, which represent fixed configuration constants for all implementations of the current version. For each class the description of the variable is followed by its name and the procedure or value which controls it. Note that variables are in lower case, while parameters are in upper case. Additional details on formats and use are presented in later sections and Appendices.

Common Variables

The following variables are common to two or more of the system, peer and packet classes. Additional variables are specific to the optional authentication mechanism as described in Appendix C. When necessary to distinguish between common variables of the same name, the variable identifier will be used.

Peer Address (peer.peeraddr, pkt.peeraddr), Peer Port (peer.peerport, pkt.peerport): These are the 32-bit Internet address and 16-bit port number of the peer.

Host Address (peer.hostaddr, pkt.hostaddr), Host Port (peer.hostport, pkt.hostport): These are the 32-bit Internet address and 16-bit port number of the host. They are included among the state variables to support multi-homing.

Leap Indicator (sys.leap, peer.leap, pkt.leap): This is a two-bit code warning of an impending leap second to be inserted in the NTP timescale. The bits are set before 23:59 on the day of insertion and reset after 00:00 on the following day. This causes the number of seconds (rollover interval) in the day of insertion to be increased or decreased by one. In the case of primary servers the bits are set by operator

intervention, while in the case of secondary servers the bits are set by the protocol. The two bits, bit 0 and bit 1 , respectively, are coded as follows :

QZ_TBL_BEG $=$ COLUMNS (2), DIMENSION (IN), COLWIDTHS (E1,E8), WIDTH $(5.0000)$, $\operatorname{ABOVE}(.0830), \operatorname{BELOW}(.0830), \operatorname{HGUTTER}(.0560), \operatorname{KEEP}(\mathrm{OFF}), \operatorname{ALIGN}(\mathrm{CT})$

QZ_TBL_BODY $=$ TABLE TEXT, TABLE TEXT

00, no warning

01, last minute has 61 seconds

10, last minute has 59 seconds

11, alarm condition (clock not synchronized)

Q Z_TBL_END $=$

In all except the alarm condition (112), NTP itself does nothing with these bits, except pass them on to the time-conversion routines that are not part of NTP. The alarm condition occurs when, for whatever reason, 
the local clock is not synchronized, such as when first coming up or after an extended period when no primary reference source is available.

Mode (peer.mode, pkt.mode): This is an integer indicating the association mode, with values coded as follows:

QZ_TBL_BEG $=$ COLUMNS (2), DIMENSION (IN), COLWIDTHS $(E 1$, E8), WIDTH $(5.0000)$, ABOVE (.0830), BELOW(.0830), HGUTTER(.0560), $\operatorname{KEEP}(\mathrm{OFF}), \operatorname{ALIGN}(\mathrm{CT})$

QZ_TBL_BODY $=$ TABLE TEXT, TABLE TEXT

0 , unspecified

1, symmetric active

2, symmetric passive

3, client

4, server

5, broadcast

6, reserved for NTP control messages

7, reserved for private use

Q Z_TBL_END $=$

Stratum (sys.stratum, peer.stratum, pkt.stratum): This is an integer indicating the stratum of the local clock, with values defined as follows :

@Z_TBL_BEG $=$ COLUMNS (2), DIMENSION (IN), COLWIDTHS (E1,E8), WIDTH $(5.0000)$, ABOVE (.0830), BELOW (.0830), HGUTTER(.0560), $\operatorname{KEEP}(\mathrm{OFF}), \operatorname{ALIGN}(\mathrm{CT})$

QZ_TBL_BODY $=$ TABLE TEXT, TABLE TEXT

0, unspecified

1, primary reference (e.g., calibrated atomic clock, radio clock)

2-255, secondary reference (via NTP)

Q Z_TBL_END $=$

For comparison purposes a value of zero is considered greater than any other value. Note that the maximum value of the integer encoded as a packet variable is limited by the parameter NTP.MAXSTRATUM.

Poll Interval (sys.poll, peer.hostpoll, peer.peerpoll, pkt.poll): This is a signed integer indicating the minimum interval between transmitted messages, in seconds as a power of two. For instance, a value of six indicates a minimum interval of 64 seconds.

Precision (sys.precision, peer.precision, pkt.precision): This is a signed integer indicating the precision of the various clocks, in seconds to the nearest power of two. The value must be rounded to the next larger power of two; for instance, a 50-Hz (20 ms) or 60-Hz (16.67 $\mathrm{ms})$ power-frequency clock would be assigned the value -5 (31.25 ms), while a 1000-Hz (1 ms) crystal-controlled clock would be assigned the value -9 (1.95 ms).

Root Delay (sys.rootdelay, peer.rootdelay, pkt.rootdelay): This is a signed fixed-point number indicating the total roundtrip delay to the primary reference source at the root of the synchronization subnet, in seconds. Note that this variable can take on both positive and negative 
values, depending on clock precision and skew.

Root Dispersion (sys.rootdispersion, peer.rootdispersion, pkt.rootdispersion): This is a signed fixed-point number indicating the maximum error relative to the primary reference source at the root of the synchronization subnet, in seconds. Only positive values greater than zero are possible.

Reference Clock Identifier (sys.refid, peer.refid, pkt.refid): This is a 32-bit code identifying the particular reference clock. In the case of stratum 0 (unspecified) or stratum 1 (primary reference source), this is a four-octet, left-justified, zero-padded ASCII string, for example (see Appendix A for comprehensive list) :

aZ_TBL_BEG $=$ COLUMNS (3), DIMENSION (IN), COLWIDTHS $(E 2, E 2, E 5)$, WIDTH (4.1700), ABOVE(.1670), BELOW(.0830), HGUTTER(.3330), $\operatorname{BOX}($ Z_SINGLE), $\operatorname{KEEP}(\mathrm{ON}), \operatorname{ALIGN}(\mathrm{CT}), \operatorname{L1}(\mathrm{R} 1 \mathrm{C} 0 \ldots \mathrm{R} 1 \mathrm{C} 3)$

QZ_TBL_BODY = TABLE CENTER, TABLE HEADER, TABLE HEADER

Stratum, Code, Meaning

@Z_TBL_BODY $=$ TABLE CENTER, TABLE TEXT, TABLE TEXT

0 , DCN, DCN routing protocol

0, TSP, TSP time protocol

1, ATOM, Atomic clock (calibrated)

1, WWVB, WWVB LF (band 5) radio

1, GOES, GOES UHF (band 9) satellite

QZ_TBL_BODY = TABLE CENTER, TABLE HEADER, TABLE HEADER

1, WWV, WWV HF (band 7) radio

Q Z_TBL_END =

In the case of stratum 2 and greater (secondary reference) this is the four-octet Internet address of the peer selected for synchronization.

Reference Timestamp (sys.reftime, peer.reftime, pkt.reftime): This is the local time, in timestamp format, when the local clock was last updated. If the local clock has never been synchronized, the value is zero.

Originate Timestamp (peer.org, pkt.org): This is the local time, in timestamp format, at the peer when its latest NTP message was sent. If the peer becomes unreachable the value is set to zero.

Receive Timestamp (peer.rec, pkt.rec): This is the local time, in timestamp format, when the latest NTP message from the peer arrived. If the peer becomes unreachable the value is set to zero.

Transmit Timestamp (peer.xmt, pkt.xmt): This is the local time, in timestamp format, at which the NTP message departed the sender.

System Variables

Table $1<\$ \& t a b 1>$ shows the complete set of system variables. In addition to the common variables described previously, the following variables are used by the operating system in order to synchronize the local clock.

Local Clock (sys.clock): This is the current local time, in timestamp 
format. Local time is derived from the hardware clock of the particular machine and increments at intervals depending on the design used. An appropriate design, including slewing and skew-Compensation mechanisms, is described in section 5.

Clock Source (sys.peer): This is a selector identifying the current synchronization source. Usually this will be a pointer to a structure containing the peer variables. The special value NULL indicates there is no currently valid synchronization source.

Peer Variables

Table 2 shows the complete set of peer variables. In addition to the common variables described previously, the following variables are used by the peer management and measurement functions.

Configured Bit (peer.config): This is a bit indicating that the association was created from configuration information and should not be demobilized if the peer becomes unreachable.

Update Timestamp (peer.update): This is the local time, in timestamp format, when the most recent NTP message was received. It is used in calculating the skew dispersion.

Reachability Register (peer.reach): This is a shift register of NTP.WINDOW bits used to determine the reachability status of the peer, with bits entering from the least significant (rightmost) end. A peer is considered reachable if at least one bit in this register is set to one.

Peer Timer (peer.timer): This is an integer counter used to control the interval between transmitted NTP messages. Once set to a nonzero value, the counter decrements at one-second intervals until reaching zero, at which time the transmit procedure is called. Note that the operation of this timer is independent of local-clock updates, which implies that the timekeeping system and interval-timer system architecture must be independent of each other. <\$\&tab2>

Packet Variables

Table 3<\$\&tab3> shows the complete set of packet variables. In addition to the common variables described previously, the following variables are defined.

Version Number (pkt.version): This is an integer indicating the version number of the sender. NTP messages will always be sent with the current version number NTP.VERSION and will always be accepted if the version number matches NTP.VERSION. Exceptions may be advised on a case-by-case basis at times when the version number is changed. Specific guidelines for interoperation between this version and previous versions of NTP are summarized in Appendix D.

Clock-Filter Variables

When the filter and selection algorithms suggested in section 4 are used, the following state variables are defined in addition to the variables described previously.

Filter Register (peer.filter): This is a shift register of NTP.SHIFT stages, where each stage stores a 3-tuple consisting of the measured delay, measured offset and calculated dispersion associated with a single observation. These 3-tuples enter from the most significant (leftmost) right and are shifted towards the least significant (rightmost) end and eventually discarded as new observations arrive.

Valid Data Counter (peer.valid): This is an integer counter indicating the valid samples remaining in the filter register. It is used to determine the reachability state and when the poll interval should be 
increased or decreased.

Offset (peer.offset): This is a signed, fixed-point number indicating the offset of the peer clock relative to the local clock, in seconds.

Delay (peer.delay): This is a signed fixed-point number indicating the roundtrip delay of the peer clock relative to the local clock over the network path between them, in seconds. Note that this variable can take on both positive and negative values, depending on clock precision and skew-error accumulation.

Dispersion (peer.dispersion): This is a signed fixed-point number indicating the maximum error of the peer clock relative to the local clock over the network path between them, in seconds. Only positive values greater than zero are possible.

Authentication Variables

When the authentication mechanism suggested in Appendix C is used, the following state variables are defined in addition to the variables described previously. These variables are used only if the optional authentication mechanism described in Appendix C is implemented.

Authentication Enabled Bit (peer.authenable): This is a bit indicating that the association is to operate in the authenticated mode.

Authenticated Bit (peer.authentic): This is a bit indicating that the last message received from the peer has been correctly authenticated.

Key Identifier (peer.hostkeyid, peer.peerkeyid, pkt.keyid): This is an integer identifying the cryptographic key used to generate the messageauthentication code.

Cryptographic Keys (sys.key): This is a set of 64-bit DES keys. Each key is constructed as in the Berkeley Unix distributions, which consists of eight octets, where the seven low-order bits of each octet correspond to the DES bits $1-7$ and the high-order bit corresponds to the DES oddparity bit 8 .

Crypto-Checksum (pkt.check): This is a crypto-checksum computed by the encryption procedure.

Parameters

Table $4<$ \& tab4> shows the parameters assumed for all implementations operating in the Internet system. It is necessary to agree on the values for these parameters in order to avoid unnecessary network overheads and stable peer associations. The following parameters are assumed fixed and applicable to all associations.

Version Number (NTP.VERSION): This is the current NTP version number (3) .

NTP Port (NTP.PORT): This is the port number (123) assigned by the Internet Assigned Numbers Authority to NTP.

Maximum Stratum (NTP.MAXSTRATUM): This is the maximum stratum value that can be encoded as a packet variable, also interpreted as <169>infinity<170> or unreachable by the subnet routing algorithm.

Maximum Clock Age (NTP.MAXAGE): This is the maximum interval a reference clock will be considered valid after its last update, in seconds.

Maximum Skew (NTP.MAXSKEW): This is the maximum offset error due to skew of the local clock over the interval determined by NTP.MAXAGE, in seconds. The ratio <\$Ephi ${ }^{\sim}{ }^{\sim}$ roman $\{$ NTP. MAXSKEW over NTP.MAXAGE $>$ is interpreted as the maximum possible skew rate due to all causes. 
Maximum Distance (NTP.MAXDISTANCE): When the selection algorithm suggested in section 4 is used, this is the maximum synchronization distance for peers acceptable for synchronization.

Minimum Poll Interval (NTP.MINPOLL): This is the minimum poll interval allowed by any peer of the Internet system, in seconds to a power of two.

Maximum Poll Interval (NTP.MAXPOLL): This is the maximum poll interval allowed by any peer of the Internet system, in seconds to a power of two.

Minimum Select Clocks (NTP.MINCLOCK): When the selection algorithm suggested in section 4 is used, this is the minimum number of peers acceptable for synchronization.

Maximum Select Clocks (NTP.MAXCLOCK): When the selection algorithm suggested in section 4 is used, this is the maximum number of peers considered for selection.

Minimum Dispersion (NTP.MINDISPERSE): When the filter algorithm suggested in section 4 is used, this is the minimum dispersion increment for each stratum level, in seconds.

Maximum Dispersion (NTP.MAXDISPERSE): When the filter algorithm suggested in section 4 is used, this is the maximum peer dispersion and the dispersion assumed for missing data, in seconds.

Reachability Register Size (NTP.WINDOW): This is the size of the reachability register (peer.reach), in bits.

Filter Size (NTP.SHIFT): When the filter algorithm suggested in section 4 is used, this is the size of the clock filter (peer.filter) shift register, in stages.

Filter Weight (NTP.FILTER): When the filter algorithm suggested in Section 4 is used, this is the weight used to compute the filter dispersion.

Select Weight (NTP.SELECT): When the selection algorithm suggested in Section 4 is used, this is the weight used to compute the select dispersion.

Modes of Operation

Except in broadcast mode, an NTP association is formed when two peers exchange messages and one or both of them create and maintain an instantiation of the protocol machine, called an association. The association can operate in one of five modes as indicated by the hostmode variable (peer.mode): symmetric active, symmetric passive, client, server and broadcast, which are defined as follows:

Symmetric Active (1): A host operating in this mode sends periodic messages regardless of the reachability state or stratum of its peer. By operating in this mode the host announces its willingness to synchronize and be synchronized by the peer.

Symmetric Passive (2): This type of association is ordinarily created upon arrival of a message from a peer operating in the symmetric active mode and persists only as long as the peer is reachable and operating at a stratum level less than or equal to the host; otherwise, the association is dissolved. However, the association will always persist until at least one message has been sent in reply. By operating in this mode the host announces its willingness to synchronize and be synchronized by the peer.

Client (3): A host operating in this mode sends periodic messages 
regardless of the reachability state or stratum of its peer. By operating in this mode the host, usually a LAN workstation, announces its willingness to be synchronized by, but not to synchronize the peer.

Server (4): This type of association is ordinarily created upon arrival of a client request message and exists only in order to reply to that request, after which the association is dissolved. By operating in this mode the host, usually a LAN time server, announces its willingness to synchronize, but not to be synchronized by the peer.

Broadcast (5): A host operating in this mode sends periodic messages regardless of the reachability state or stratum of the peers. By operating in this mode the host, usually a LAN time server operating on a high-speed broadcast medium, announces its willingness to synchronize all of the peers, but not to be synchronized by any of them.

A host operating in client mode occasionally sends an NTP message to a host operating in server mode, perhaps right after rebooting and at periodic intervals thereafter. The server responds by simply interchanging addresses and ports, filling in the required information and returning the message to the client. Servers need retain no state information between client requests, while clients are free to manage the intervals between sending NTP messages to suit local conditions. In these modes the protocol machine described in this document can be considerably simplified to a simple remote-procedure-call mechanism without significant loss of accuracy or robustness, especially when operating over high-speed LANs.

In the symmetric modes the client/server distinction (almost)

disappears. Symmetric passive mode is intended for use by time servers operating near the root nodes (lowest stratum) of the synchronization subnet and with a relatively large number of peers on an intermittent basis. In this mode the identity of the peer need not be known in advance, since the association with its state variables is created only when an NTP message arrives. Furthermore, the state storage can be reused when the peer becomes unreachable or is operating at a higher stratum level and thus ineligible as a synchronization source.

Symmetric active mode is intended for use by time servers operating near the end nodes (highest stratum) of the synchronization subnet. Reliable time service can usually be maintained with two peers at the next lower stratum level and one peer at the same stratum level, so the rate of ongoing polls is usually not significant, even when connectivity is lost and error messages are being returned for every poll.

Normally, one peer operates in an active mode (symmetric active, client or broadcast modes) as configured by a startup file, while the other operates in a passive mode (symmetric passive or server modes), often without prior configuration. However, both peers can be configured to operate in the symmetric active mode. An error condition results when both peers operate in the same mode, but not symmetric active mode. In such cases each peer will ignore messages from the other, so that prior associations, if any, will be demobilized due to reachability failure.

Broadcast mode is intended for operation on high-speed LANs with numerous workstations and where the highest accuracies are not required. In the typical scenario one or more time servers on the LAN send periodic broadcasts to the workstations, which then determine the time on the basis of a preconfigured latency in the order of a few milliseconds. As in the client/server modes the protocol machine can be considerably simplified in this mode; however, a modified form of the clock selection algorithm may prove useful in cases where multiple time servers are used for enhanced reliability.

Event Processing

The significant events of interest in NTP occur upon expiration of a 
peer timer (peer.timer), one of which is dedicated to each peer with an active association, and upon arrival of an NTP message from the various peers. An event can also occur as the result of an operator command or detected system fault, such as a primary reference source failure. This section describes the procedures invoked when these events occur.

\section{Notation Conventions}

The NTP filtering and selection algorithms act upon a set of variables for clock offset (<\$Etheta, $\sim$ THETA $>$ ), roundtrip delay (<\$Edelta , $\sim$ DELTA $>$ ) and dispersion (<\$Eepsilon, $\sim$ EPSILON $>$ ). When necessary to distinguish between them, lower-case Greek letters are used for variables relative to a peer, while upper-case Greek letters are used for variables relative to the primary reference source(s), i.e., via the peer to the root of the synchronization subnet. Subscripts will be used to identify the particular peer when this is not clear from context. The algorithms are based on a quantity called the synchronization distance (<\$Elambda, LAMBDA $>$ ), which is computed from the roundtrip delay and dispersion as described below.

As described in Appendix $\mathrm{H}$, the peer dispersion <\$Eepsilon> includes contributions due to measurement error $<\$ E_{r h o}{ }^{\sim} 1^{\sim}<<<<$ roman sys.precision>, skew-error accumulation <\$Ephi tau>, where $<$ Ephi ${ }^{\sim}$ roman $\{$ NTP. MAXSKEW over NTP.MAXAGE\}> is the maximum skew rate and <\$Etau ${ }^{\sim}$ roman $\left\{\right.$ sys.clock ${ }^{\sim} \sim$ peer.update\}> is the interval since the last update, and filter (sample) dispersion <\$Eepsilon sub sigma> computed by the clock-filter algorithm. The root dispersion <\$EEPSILON> includes contributions due to the selected peer dispersion <\$Eepsilon> and skew-error accumulation <\$Ephi tau>, together with the root dispersion for the peer itself. The system dispersion includes the select (sample) dispersion <\$Eepsilon sub xi> computed by the clockselect algorithm and the absolute initial clock offset <\$E $\mid$ THETA |> provided to the local-clock algorithm. Both <\$Eepsilon> and <\$EEPSILON> are dynamic quantities, since they depend on the elapsed time <\$Etau> since the last update, as well as the sample dispersions calculated by the algorithms.

Each time the relevant peer variables are updated, all dispersions associated with that peer are updated to reflect the skew-error accumulation. The computations can be summarized as follows:

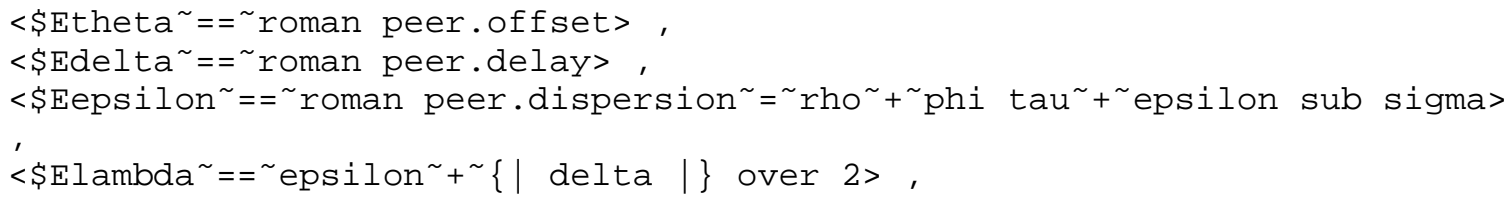

where <\$Etau> is the interval since the original timestamp (from which $<$ Stheta> and <\$Edelta> were determined) was transmitted to the present time and <\$Eepsilon sub sigma> is the filter dispersion (see clockfilter procedure below). The variables relative to the root of the synchronization subnet via peer $i$ are determined as follows:

$<$ ETHETA sub $i^{\sim}==^{\sim}$ theta sub $i>$, $<$ \$EDLTA sub $i^{\sim}==^{\sim}$ roman peer. rootdelay ${ }^{\sim}$ delta sub i>, $<$ SEPSILON sub $i^{\sim}==^{\sim}$ roman peer. rootdispersion ${ }^{\sim}{ }^{\sim}$ epsilon sub $i^{\sim}+{ }^{\sim}$ phi tau sub i>, $<$ SLAMBDA sub $i^{\sim}==^{\sim}$ EPSILON sub $i^{\sim}+\sim \mid$ DELTA sub $\left.i \mid\right\}$ over $2>$,

where all variables are understood to pertain to the ith peer. Finally, assuming the ith peer is selected for synchronization, the system variables are determined as follows:

$<$ SETHET $\sim{ }^{\sim}>$ combined final offset,

$<$ EDELTA $\sim{ }^{\sim}$ DELTA sub i>,

$<$ \$EEPSILON ${ }^{\sim}$ EPSILON sub $i^{\sim}+\sim$ epsilon sub $\mathrm{xi} \sim{ }^{\sim}|\operatorname{THETA}|>$,

$<$ ELAMBDA ${ }^{\sim}=\sim$ LAMBDA sub i>, 
where <\$Eepsilon sub $\mathrm{xi}>$ is the select dispersion (see clock-selection procedure below).

Informal pseudo-code which accomplishes these computations is presented below. Note that the pseudo-code is represented in no particular language, although it has many similarities to the C language. Specific details on the important algorithms are further illustrated in the Clanguage routines in Appendix I.

Transmit Procedure

The transmit procedure is executed when the peer timer decrements to zero for all modes except client mode with a broadcast server and server mode in all cases. In client mode with a broadcast server messages are never sent. In server mode messages are sent only in response to received messages. This procedure is also called by the receive procedure when an NTP message arrives that does not result in a persistent association.

begin transmit procedure

The following initializes the packet buffer and copies the packet variables. The value skew is necessary to account for the skew-error accumulated over the interval since the local clock was last set.

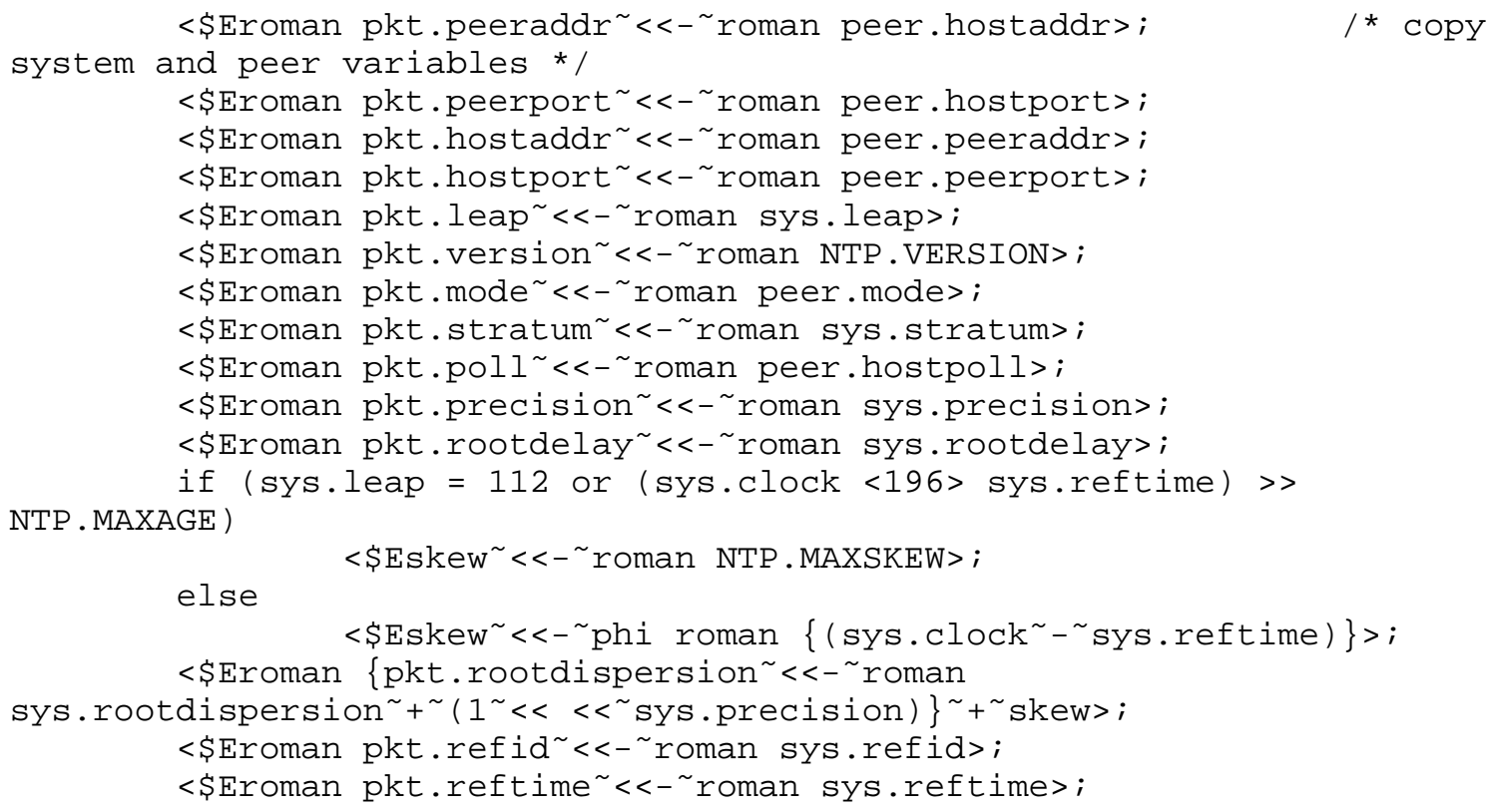

The transmit timestamp pkt.xmt will be used later in order to validate the reply; thus, implementations must save the exact value transmitted. In addition, the order of copying the timestamps should be designed so that the time to format and copy the data does not degrade accuracy.

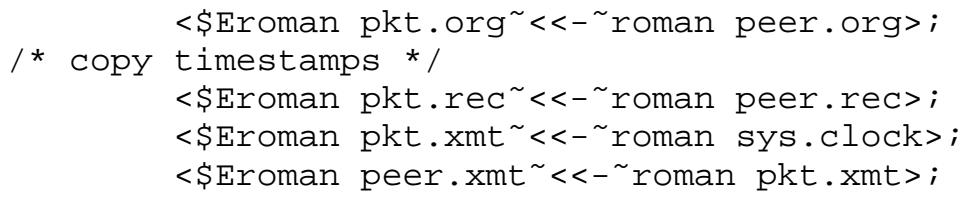

The call to encrypt is implemented only if authentication is implemented. If authentication is enabled, the delay to encrypt the authenticator may degrade accuracy. Therefore, implementations should include a system state variable (not mentioned elsewhere in this specification) which contains an offset calculated to match the expected encryption delay and correct the transmit timestamp as obtained from the local clock. 
procedure succeed. If, in addition, tests one through four succeed (valid data), the clock-update procedure is called to update the local clock. Otherwise, if the association was not previously configured, an immediate reply is sent and the association demobilized.

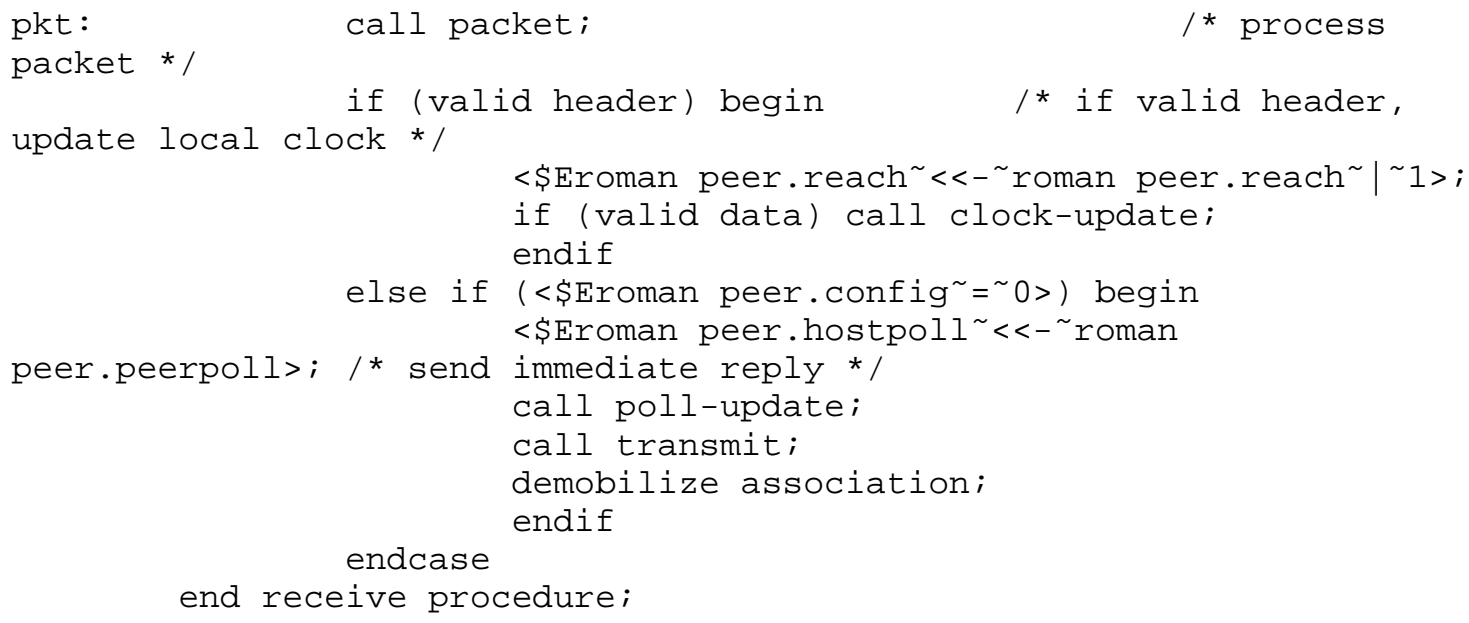

Packet Procedure

The packet procedure checks the message validity, computes delay/offset samples and calls other procedures to filter the data and select the synchronization source. Test 1 requires the transmit timestamp not match the last one received from the same peer; otherwise, the message might be an old duplicate. Test 2 requires the originate timestamp match the last one sent to the same peer; otherwise, the message might be out of order, bogus or worse. In case of broadcast mode (5) the apparent roundtrip delay will be zero and the full accuracy of the time-transfer operation may not be achievable. However, the accuracy achieved may be adequate for most purposes. The poll-update procedure is called with argument peer.hostpoll (peer.peerpoll may have changed).

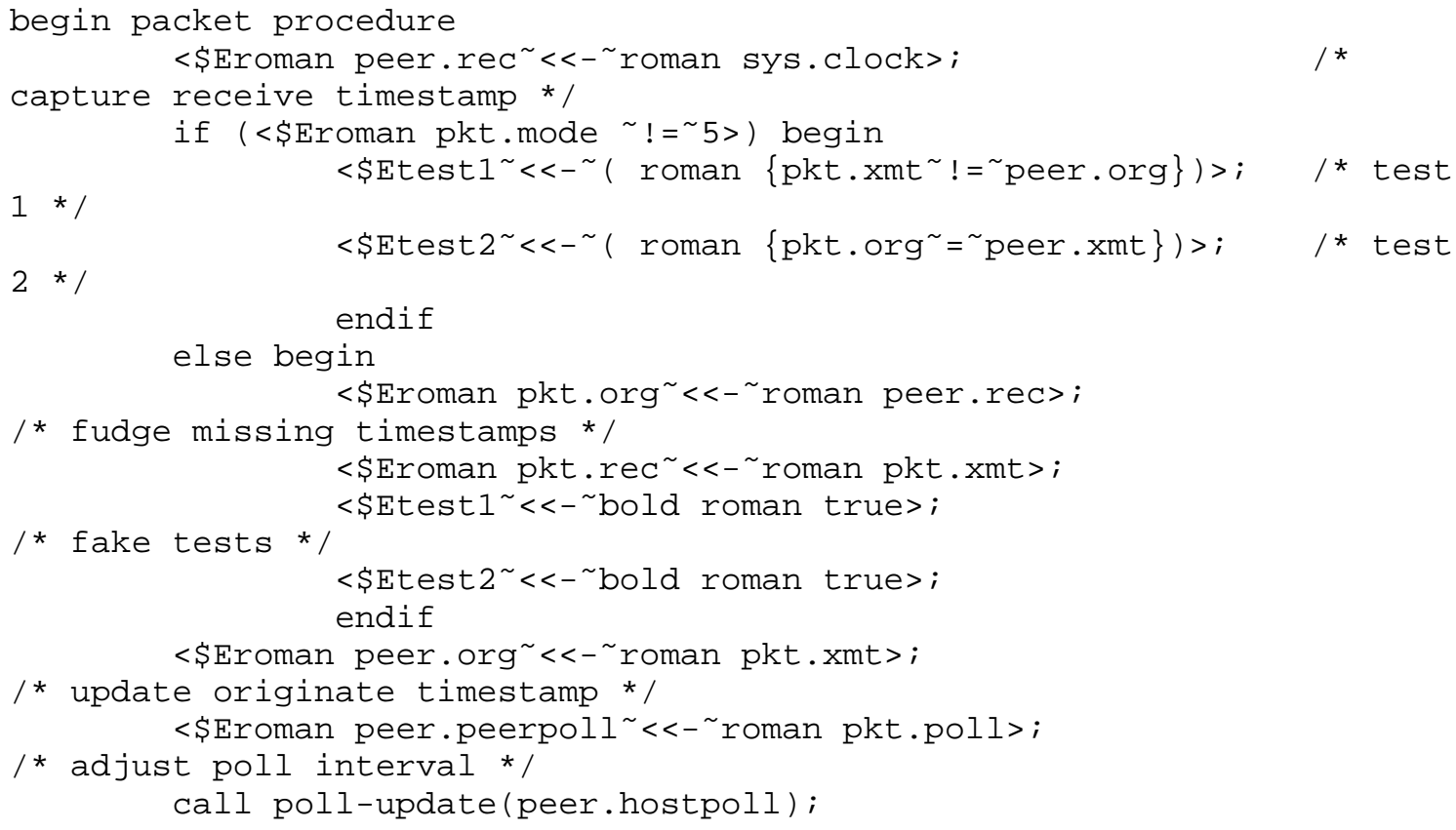

Test 3 requires that both the originate and receive timestamps are nonzero. If either of the timestamps are zero, the association has not synchronized or has lost reachability in one or both directions.

$<$ Etest $3^{\sim}<<-^{\sim}\left(\operatorname{roman}\right.$ pkt.org ! $^{\sim} 0>$ and $<$ Eroman pkt.rec ! $\left.^{\sim} 0\right)>$; $1 *$ test $3 * 1$

The roundtrip delay and clock offset relative to the peer are calculated 
as follows. Number the times of sending and receiving NTP messages as shown in Figure $2<\$ \& f i g 2>$ and let $i$ be an even integer. Then Ti-3, Ti-2, Ti-1 and $\mathrm{Ti}$ are the contents of the pkt.org, pkt.rec, pkt.xmt and peer.rec variables, respectively. The clock offset <\$Etheta>, roundtrip delay <\$Edelta> and dispersion <\$Eepsilon> of the host relative to the peer is:

$<$ Edelta ${ }^{\sim}\left(\mathrm{T} \text { sub } i^{\sim}-^{\sim} \mathrm{T} \text { sub }\{i-3\}\right)^{\sim}-^{\sim}\left(\mathrm{T}\right.$ sub $\{i-1\}^{\sim}-^{\sim} \mathrm{T}$ sub $\{i-2\}$ ) , <\$Ethet ${ }^{\sim}=^{\sim}\left\{\left(\mathrm{T} \text { sub }\{i-2\}^{\sim}-^{\sim} \mathrm{T} \text { sub }\{i-3\}\right)^{\sim}++^{\sim}\left(\mathrm{T}\right.\right.$ sub $\{i-1\} \sim-^{\sim} \mathrm{T}$ sub $\left.\left.i\right)\right\}$ over $2>$,

$<$ Eepsilon ${ }^{\sim} \sim \operatorname{roman} \quad\left\{\left(1^{\sim}<<<<\text { sys.precision }\right\}\right)^{\sim}+\sim \operatorname{phi}\left(\mathrm{T}\right.$ sub $i{ }^{\sim}{ }^{\sim} \mathrm{T}$ sub $\{i-$ $3\})>$,

where, as before, <\$Ephi ${ }^{\sim}$ roman $\{$ NTP.MAXSKEW over NTP.MAXAGE $\}>$. The quantity <\$Eepsilon> represents the maximum error or dispersion due to measurement error at the host and local-clock skew accumulation over the interval since the last message was transmitted to the peer. Subsequently, the dispersion will be updated by the clock-filter procedure.

The above method amounts to a continuously sampled, returnable-time system, which is used in some digital telephone networks [BEL86]. Among the advantages are that the order and timing of the messages are unimportant and that reliable delivery is not required. Obviously, the accuracies achievable depend upon the statistical properties of the outbound and inbound data paths. Further analysis and experimental results bearing on this issue can be found in [MIL90] and in Appendix $H$. Test 4 requires that the calculated delay be within <169>reasonable<170> bounds:

\section{$<$ \$Etest $4^{\sim}<<-\sim\left(\mid\right.$ delta $\mid \sim<<^{\sim}$ roman NTP. MAXDISPERSE ${ }^{\sim}$ bold and $\sim$ epsilon $\sim<\sim$ roman NTP.MAXDISPERSE) $>$; /* test 4 * /}

Test 5 is implemented only if the authentication mechanism described in Appendix C is implemented. It requires either that authentication be explicitly disabled or that the authenticator be present and correct as determined by the decrypt procedure.

$$
\begin{aligned}
& \text { \#ifdef (authentication implemented) / test } 5 \text { * / } \\
& <\text { \$Etest } 5^{\sim}<-^{\sim} \text { ( roman }\left\{\text { (peer. config }{ }^{\sim}=1^{\sim}\right. \text { bold }
\end{aligned}
$$

and peer. authenable ${ }^{\sim}{ }^{\sim} 0$ ) $\sim$ bold or or $^{\sim}$ peer. authentic $\left.\left.{ }^{\sim}{ }^{\sim} 1\right\}\right)>$; \#endef

Test 6 requires the peer clock be synchronized and the interval since the peer clock was last updated be positive and less than NTP. MAXAGE. Test 7 insures that the host will not synchronize on a peer with greater stratum. Test 8 requires that the header contains <169>reasonable<170> values for the pkt.rootdelay and pkt.rootdispersion fields.

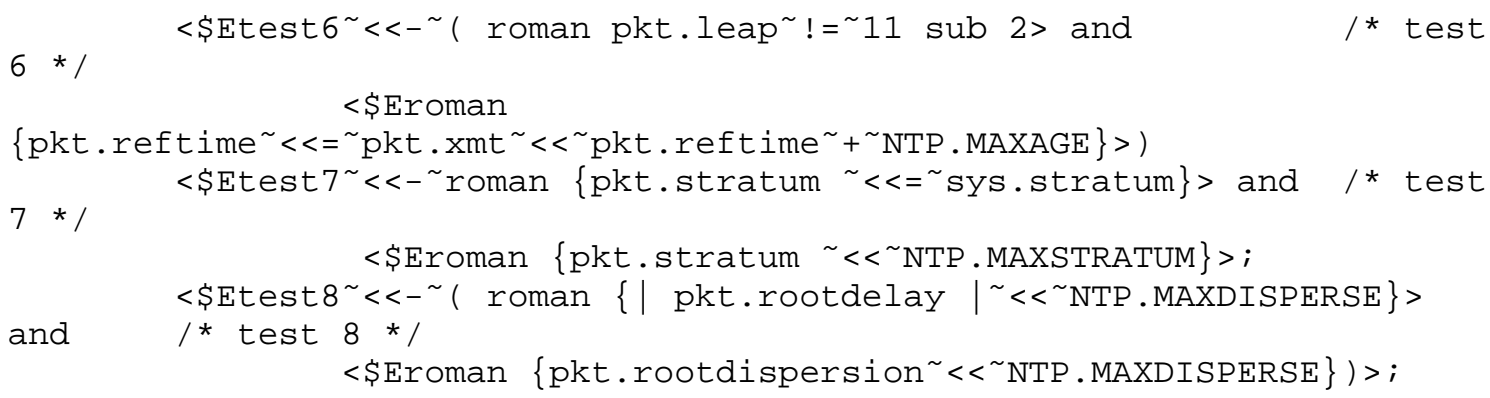

and

$<$ Eroman \{pkt.stratum $\sim<\sim$ NTP. MAXSTRATUM $\}$; $<$ Stest $8^{\sim}<-^{\sim}\left(\operatorname{roman}\left\{\mid\right.\right.$ pkt. rootdelay $\mid \sim<<^{\sim N T P}$. MAXDISPERSE $\}>$ $1 *$ test $8 * /$ $<$ Eroman \{pkt.rootdispersion ${ }^{2}<<^{\sim}$ NTP.MAXDISPERSE\}) $>$;

With respect to further processing, the packet includes valid (synchronized) data if tests one through four succeed

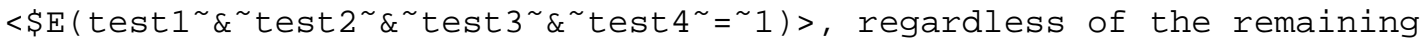
tests. Only packets with valid data can be used to calculate offset, delay and dispersion values. The packet includes a valid header if tests five through eight succeed $\left\langle \$ E\left(\right.\right.$ test5 \& $^{\sim}$ test $6^{\sim}$ \& $^{\sim}$ test $7^{\sim}$ \& $^{\sim}$ test $\left.\left.8^{\sim}=\sim 1\right)\right\rangle$, 
regardless of the remaining tests. Only packets with valid headers can be used to determine whether a peer can be selected for synchronization. Note that <\$Etest $1>$ and <\$Etest2> are not used in broadcast mode (forced to true), since the originate and receive timestamps are undefined.

The clock-filter procedure is called to produce the delay (peer.delay), offset (peer.offset) and dispersion (peer.dispersion) for the peer. Specification of the clock-filter algorithm is not an integral part of the NTP specification, since there may be other algorithms that work well in practice. However, one found to work well in the Internet environment is described in section 4 and its use is recommended.

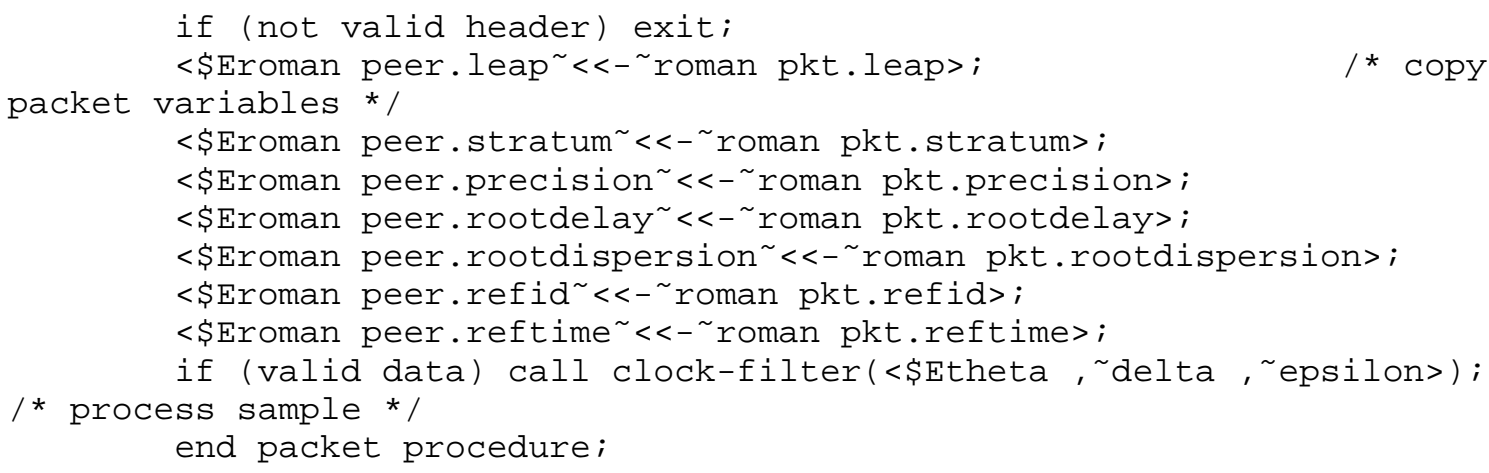

It may happen that the local clock may be reset, rather than slewed to its final value. In this case the clear procedure is called for every peer to purge the clock filter, reset the poll interval and reselect the synchronization source, if necessary. Note that the local-clock procedure sets the leap bits sys.leap to $<169>$ unsynchronized<170> 112 in this case, so that no other peer will attempt to synchronize to the host until the host once again selects a peer for synchronization.

The distance procedure calculates the root delay <\$EDELTA>, root dispersion <\$EEPSILON> and root synchronization distance <\$ELAMBDA> via the peer to the root of the synchronization subnet. The host will not synchronize to the selected peer if the distance is greater than NTP.MAXDISTANCE. The reason for the minimum clamp at NTP.MINDISPERSE is to discourage subnet route flaps that can happen with Bellman-Ford algorithms and small roundtrip delays.

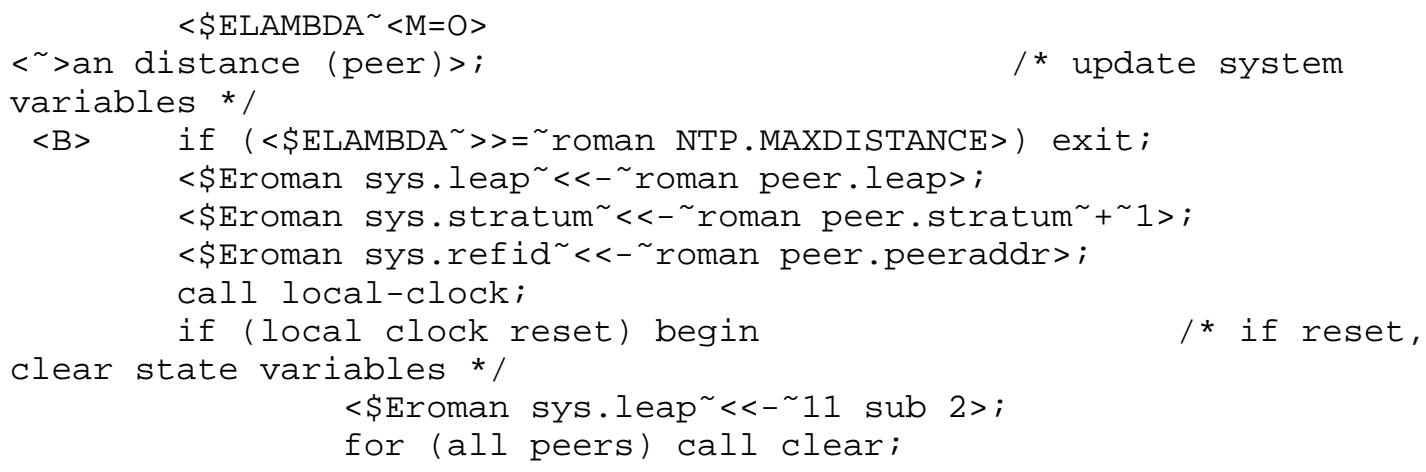




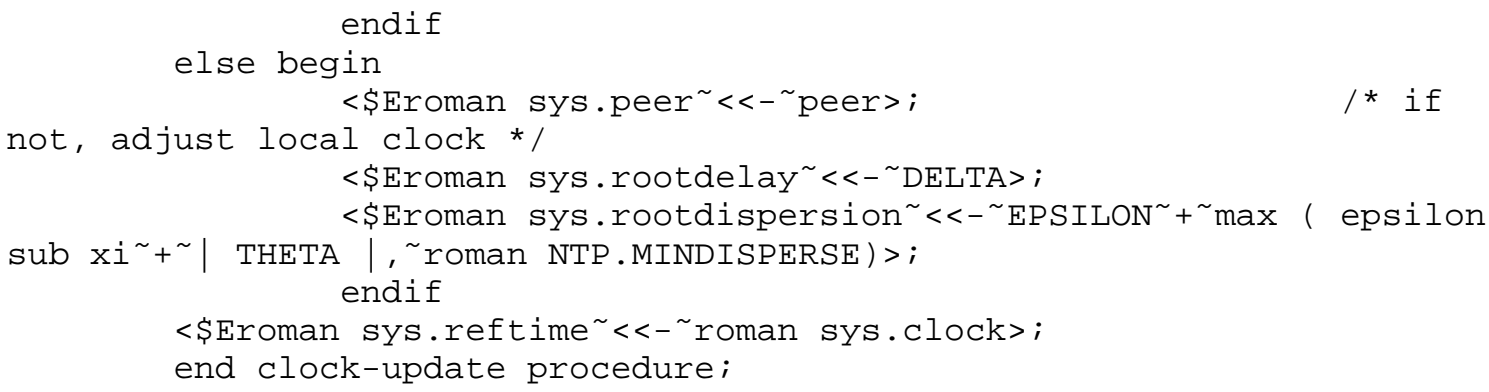

In some system configurations a precise source of timing information is available in the form of a train of timing pulses spaced at one-second intervals. Usually, this is in addition to a source of timecode information, such as a radio clock or even NTP itself, to number the seconds, minutes, hours and days. In these configurations the system variables are set to refer to the source from which the pulses are derived. For those configurations which support a primary reference source, such as a radio clock or calibrated atomic clock, the stratum is set at one as long as this is the actual synchronization source and whether or not the primary-clock procedure is used.

Specification of the clock-selection and local-clock algorithms is not an integral part of the NTP specification, since there may be other algorithms which provide equivalent performance. However, a clockselection algorithm found to work well in the Internet environment is described in section 4, while a local-clock algorithm is described in Section 5 and their use is recommended. The clock-selection algorithm described in section 4 usually picks the peer at the lowest stratum and minimum synchronization distance among all those available, unless that peer appears to be a falseticker. The result is that the algorithms all work to build a minimum-weight spanning tree relative to the primary reference time servers and thus a hierarchical-master-slave synchronization subnet.

Primary-Clock Procedure

When a primary reference source such as a radio clock is connected to the host, it is convenient to incorporate its information into the data base as if the clock were represented as an ordinary peer. In the primary-clock procedure the clock is polled once a minute or so and the returned timecode used to produce a new update for the local clock. When peer.timer decrements to zero for a primary clock peer, the transmit procedure is not called; rather, the radio clock is polled, usually using an ASCII string specified for this purpose. When a valid timecode is received from the radio clock, it is converted to NTP timestamp format and the peer variables updated. The value of peer.leap is set depending on the status of the leap-warning bit in the timecode, if available, or manually by the operator. The value for peer.peeraddr, which will become the value of sys.refid when the clock-update procedure is called, is set to an ASCII string describing the clock type (see Appendix A).

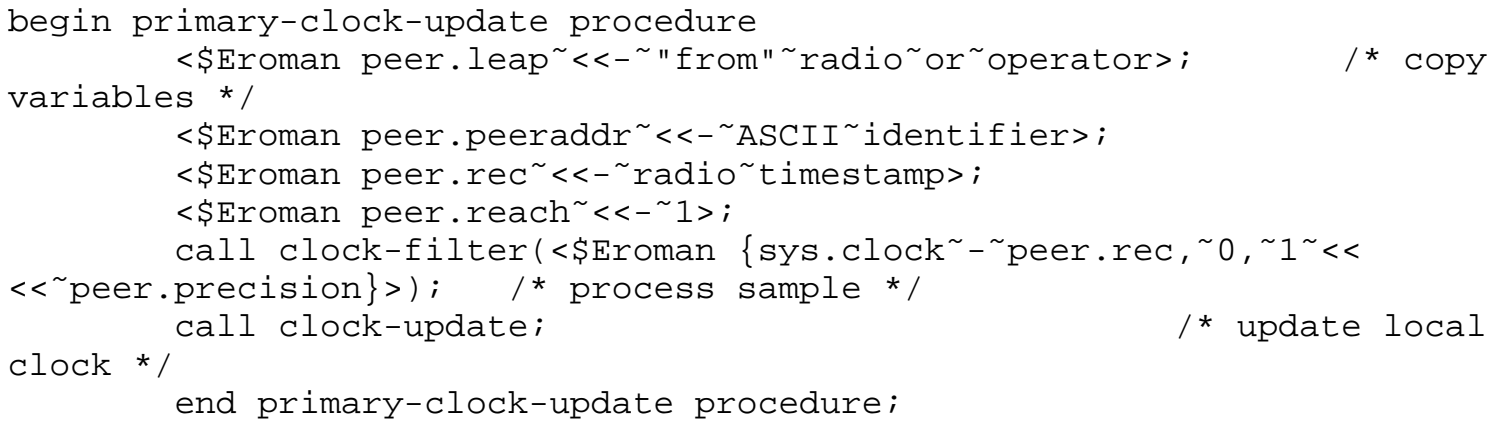

Initialization Procedures 
The initialization procedures are used to set up and initialize the system, its peers and associations.

\section{Initialization Procedure}

The initialization procedure is called upon reboot or restart of the NTP daemon. The local clock is presumably undefined at reboot; however, in some equipment an estimate is available from the reboot environment, such as a battery-backed clock/calendar. The precision variable is determined by the intrinsic architecture of the local hardware clock. The authentication variables are used only if the authentication mechanism described in Appendix C is implemented. The values of these variables are determined using procedures beyond the scope of NTP itself.

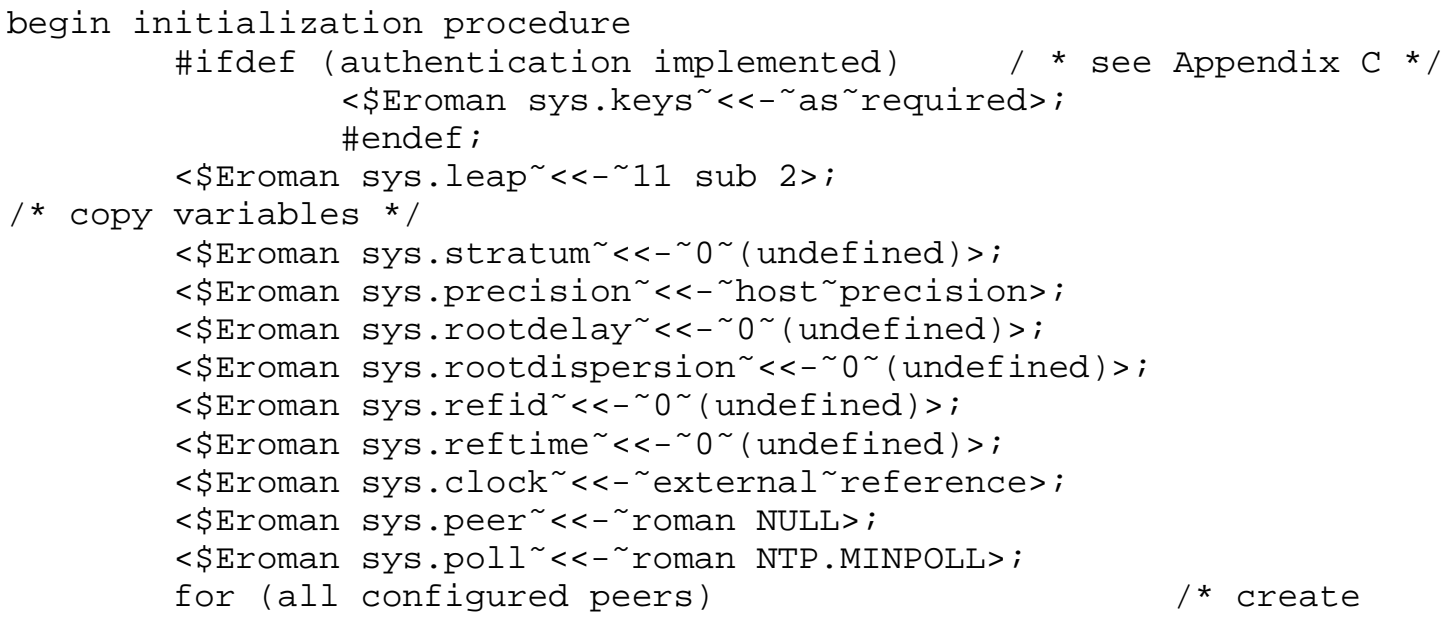

Initialization-Instantiation Procedure

This implementation-specific procedure is called from the initialization procedure to define an association. The addresses and modes of the peers are determined using information read during the reboot procedure or as the result of operator commands. The authentication variables are used only if the authentication mechanism described in Appendix $C$ is implemented. The values of these variables are determined using procedures beyond the scope of NTP itself. With the authentication bits set as suggested, only properly authenticated peers can become the synchronization source.

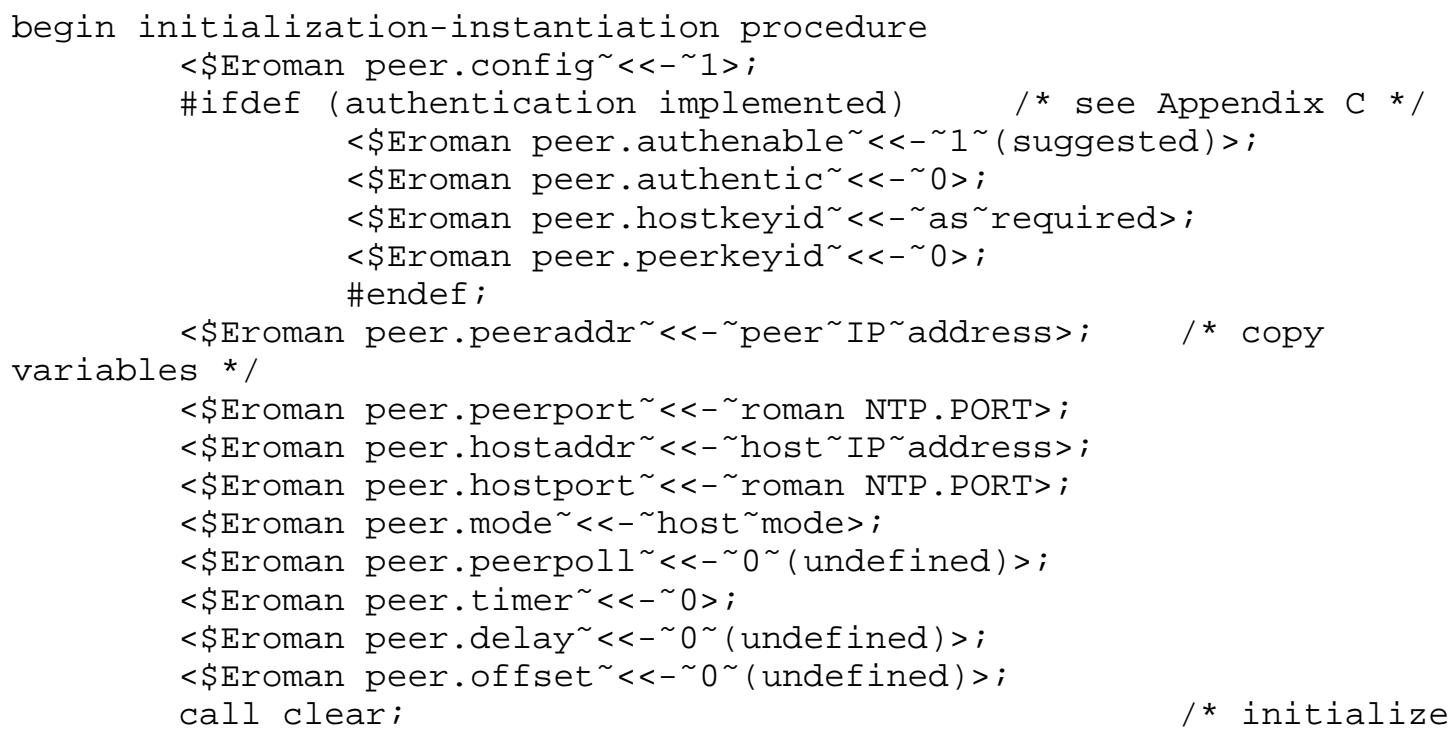


end initialization-instantiation procedure;

\section{Receive-Instantiation Procedure}

The receive-instantiation procedure is called from the receive procedure when a new peer is discovered. It initializes the peer variables and mobilizes the association. If the message is from a peer operating in client mode (3), the host mode is set to server mode (4); otherwise, it is set to symmetric passive mode (2). The authentication variables are used only if the authentication mechanism described in Appendix $C$ is implemented. If implemented, only properly authenticated non-configured peers can become the synchronization source.

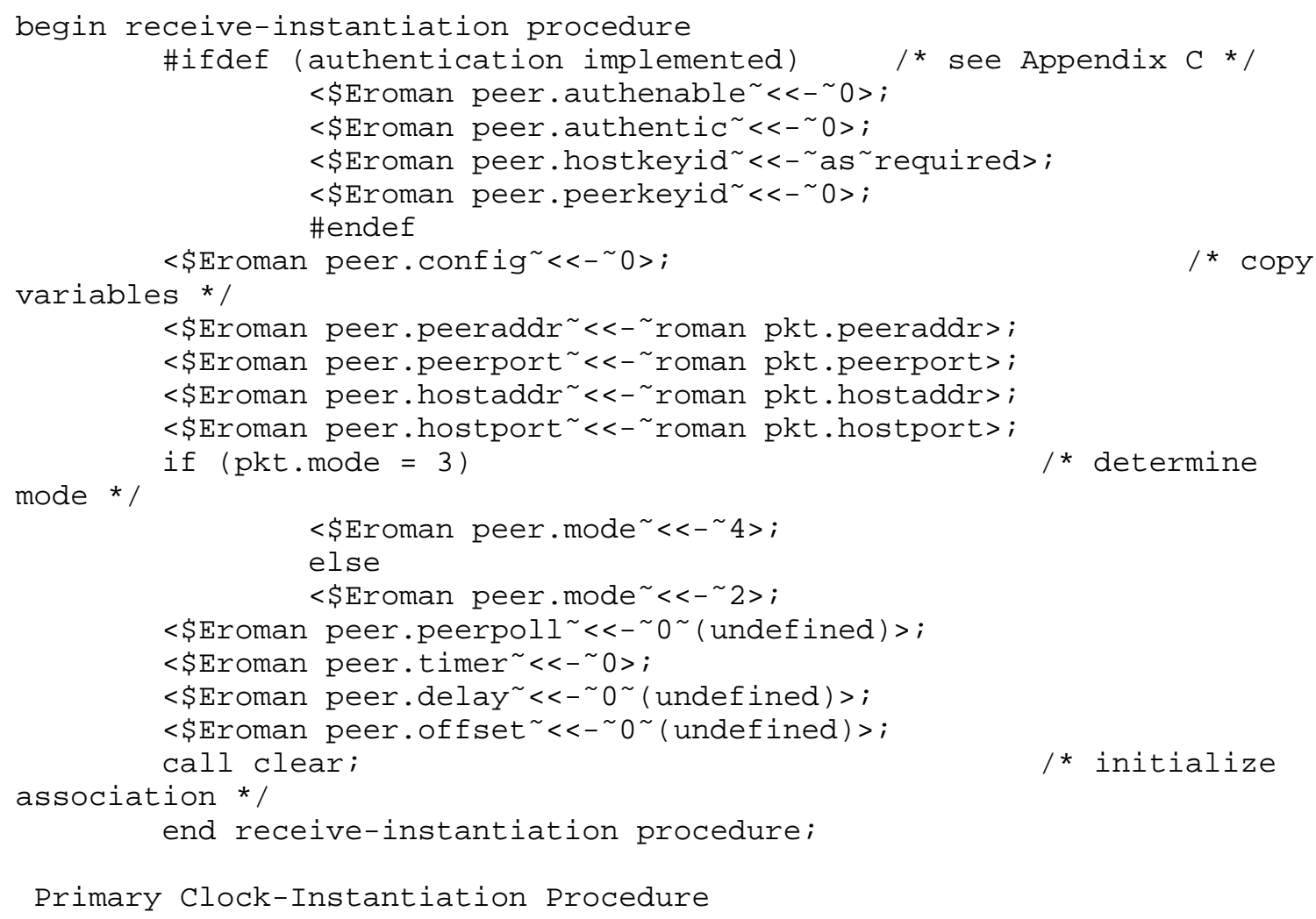

This procedure is called from the initialization procedure in order to set up the state variables for the primary clock. The value for peer.precision is determined from the radio clock specification and hardware interface. The value for peer.rootdispersion is nominally ten times the inherent maximum error of the radio clock; for instance, $<\$ E 10 \sim \mathrm{mu} s>$ for a calibrated atomic clock, $10 \mathrm{~ms}$ for a WWVB or GOES radio clock and $100 \mathrm{~ms}$ for a less accurate WWV radio clock.

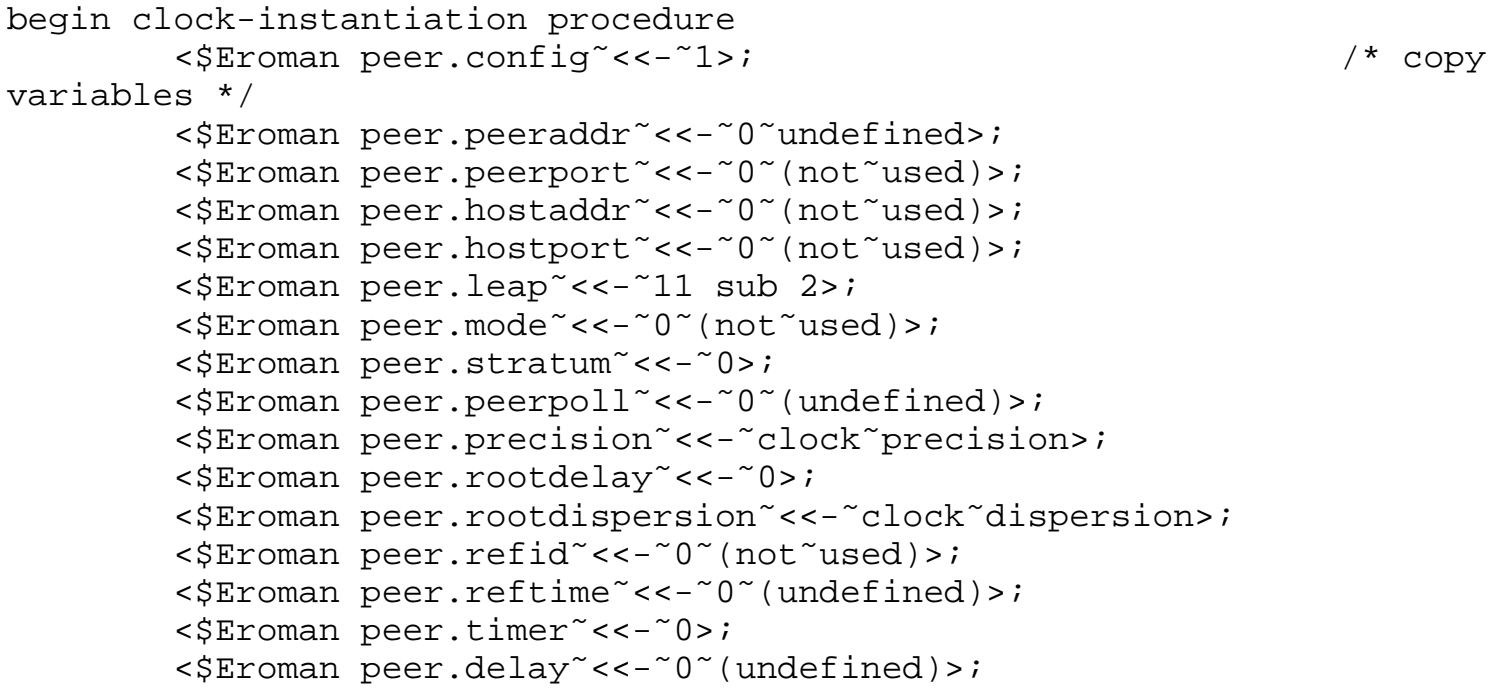




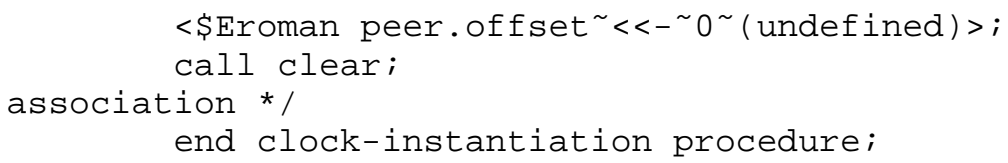

In some configurations involving a calibrated atomic clock or LORAN-C receiver, the primary reference source may provide only a seconds pulse, but lack a complete timecode from which the numbering of the seconds, etc., can be derived. In these configurations seconds numbering can be derived from other sources, such as a radio clock or even other NTP peers. In these configurations the primary clock variables should reflect the primary reference source, not the seconds-numbering source; however, if the seconds-numbering source fails or is known to be operating incorrectly, updates from the primary reference source should be suppressed as if it had failed.

\section{Clear Procedure}

The clear procedure is called when some event occurs that results in a significant change in reachability state or potential disruption of the local clock.

Poll-Update Procedure

The poll-update procedure is called when a significant event occurs that may result in a change of the poll interval or peer timer. It checks the values of the host poll interval (peer.hostpoll) and peer poll interval (peer.peerpoll) and clamps each within the valid range. If the peer is selected for synchronization, the value is further clamped as a function of the computed compliance (see section 5).

begin poll-update procedure

$<$ Stemp $\sim<-\sim$ roman peer.hostpoll>; /*

determine host poll interval */

if $\left(<\right.$ Epeer ${ }^{\sim}{ }^{\sim}$ roman sys.peer $\left.>\right)$

else $<$ Stemp $\sim<-\sim \min$ (temp, $\sim \operatorname{roman}$ \{sys.poll, $\sim$ NTP. MAXPOLL) \} $>$; $<$ SEtemp $\sim<-\sim \min$ (temp, $\sim$ roman NTP. MAXPOLL) $>$;

$<$ Eroman peer.hostpoll $\sim<-\sim \max$ (temp, $\sim \operatorname{roman}$ NTP.MINPOLL) $>$; $<$ Stemp $\sim<-\sim 1 \sim<<<$ min ( roman fpeer.hostpoll, $\sim \max$

(peer.peerpoll, NTP.MINPOLL) \}) >;

If the poll interval is unchanged and the peer timer is zero, the timer is simply reset. If the poll interval is changed and the new timer value is greater than the present value, no additional action is necessary; otherwise, the peer timer must be reduced. When the peer timer must be reduced it is important to discourage tendencies to synchronize transmissions between the peers. A prudent precaution is to randomize the first transmission after the timer is reduced, for instance by the sneaky technique illustrated. 
timer */

if (peer.timer $=0$ )

/* reset peer

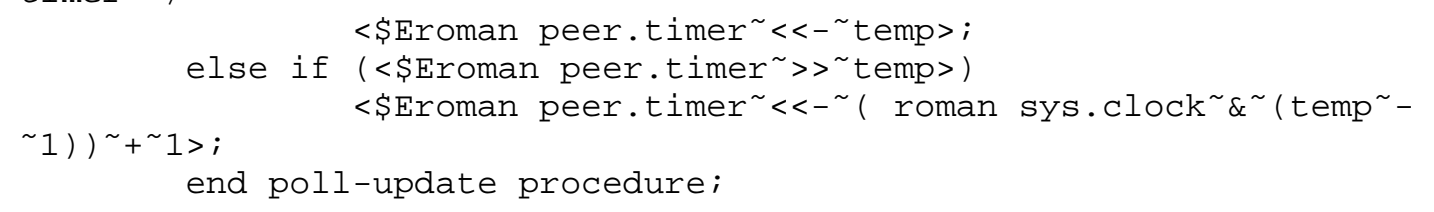

Synchronization Distance Procedure

The distance procedure calculates the synchronization distance from the peer variables for the peer peer.

begin distance(peer) procedure; $<$ \$EDELT $\sim<-\sim$ roman $\left\{\right.$ peer.rootdelay ${ }^{\sim} \sim \mid$ peer.delay $\left.\mid\right\}>$; $<$ SEPSILON $\sim<-\sim$ roman

\{peer.rootdispersion ${ }^{+}{ }^{\sim}$ peer.dispersion ${ }^{\sim}{ }^{\sim}$ phi (sys.clock ${ }^{\sim}{ }^{\sim}$ peer.update) \}$>$; $<$ SLAMBDA $\sim<-\sim$ EPSILON ${ }^{*} \sim\{|\operatorname{DELTA}|\}$ over 2> ; end distance procedure;

Note that, while <\$EDELTA> may be negative in some cases, both $<$ \$EPSILON> and <\$ELAMBDA> are always positive.

\section{Access Control Issues}

The NTP design is such that accidental or malicious data modification (tampering) or destruction (jamming) at a time server should not in general result in timekeeping errors elsewhere in the synchronization subnet. However, the success of this approach depends on redundant time servers and diverse network paths, together with the assumption that tampering or jamming will not occur at many time servers throughout the synchronization subnet at the same time. In principle, the subnet vulnerability can be engineered through the selection of time servers known to be trusted and allowing only those time servers to become the synchronization source. The authentication procedures described in Appendix $C$ represent one mechanism to enforce this; however, the encryption algorithms can be quite CPU-intensive and can seriously degrade accuracy, unless precautions such as mentioned in the description of the transmit procedure are taken.

While not a required feature of NTP itself, some implementations may include an access-control feature that prevents unauthorized access and controls which peers are allowed to update the local clock. For this purpose it is useful to distinguish between three categories of access: those that are preauthorized as trusted, preauthorized as friendly and all other (non-preauthorized) accesses. Presumably, preauthorization is accomplished by entries in the configuration file or some kind of ticket-management system such as Kerberos [STE88]. In this model only trusted accesses can result in the peer becoming the synchronization source. While friendly accesses cannot result in the peer becoming the synchronization source, NTP messages and timestamps are returned as specified.

It does not seem useful to maintain a secret clock, as would result from restricting non-preauthorized accesses, unless the intent is to hide the existence of the time server itself. Well-behaved Internet hosts are expected to return an ICMP service-unavailable error message if a service is not implemented or resources are not available; however, in the case of NTP the resources required are minimal, so there is little need to restrict requests intended only to read the clock. A simple but effective access-control mechanism is then to consider all associations preconfigured in a symmetric mode or client mode (modes 1,2 and 3 ) as trusted and all other associations, preconfigured or not, as friendly. 
on an access-control list with each entry consisting of a 32-bit

Internet address, 32-bit mask and three-bit mode. If the logical AND of the source address (pkt.peeraddr) and the mask in an entry matches the corresponding address in the entry and the mode (pkt.mode) matches the mode in the entry, the access is allowed; otherwise an ICMP error message is returned to the requestor. Through appropriate choice of mask, it is possible to restrict requests by mode to individual addresses, a particular subnet or net addresses, or have no restriction at all. The access-control list would then serve as a filter controlling which peers could create associations.

Filtering and Selection Algorithms

A most important factor affecting the accuracy and reliability of time distribution is the complex of algorithms used to reduce the effect of statistical errors and falsetickers due to failure of various subnet components, reference sources or propagation media. The algorithms suggested in this section were developed and refined over several years of operation in the Internet under widely varying topologies, speeds and traffic regimes. While these algorithms are believed the best available at the present time, they are not an integral part of the NTP specification, since other algorithms with similar or superior performance may be devised in future.

However, it is important to observe that not all time servers or clients in an NTP synchronization subnet must implement these algorithms. For instance, simple workstations may dispense with one or both of them in the interests of simplicity if accuracy and reliability requirements justify. Nevertheless, it would be expected that an NTP server providing synchronization to a sizable community, such as a university campus or research laboratory, would be expected to implement these algorithms or others proved to have equivalent functionality. A comprehensive discussion of the design principles and performance is given in [MIL91a].

In order for the NTP filter and selection algorithms to operate effectively, it is useful to have a measure of recent sample variance recorded for each peer. The measure adopted is based on first-order differences, which are easy to compute and effective for the purposes intended. There are two measures, one called the filter dispersion $<$ Eepsilon sub sigma> and the other the select dispersion <\$Eepsilon sub xi>. Both are computed as the weighted sum of the clock offsets in a temporary list sorted by synchronization distance. If <\$Etheta sub i $\sim\left(0^{\sim}<<=i^{\sim}<<^{\sim} n\right)>$ is the offset of the ith entry, then the sample difference <\$Eepsilon sub $i j>$ of the ith entry relative to the jth entry is defined $<$ Eepsilon sub $i j^{\sim}\left\langle{ }^{\sim}>=^{\sim}\right|$ theta sub $i^{\sim}-^{\sim}$ theta sub $j \mid>$. The dispersion relative to the jth entry is defined <\$Eepsilon sub $j>$ and computed as the weighted sum

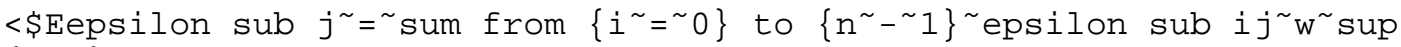
$\{i+1\}>$,

where $w$ is a weighting factor chosen to control the influence of synchronization distance in the dispersion budget. In the NTP algorithms $\mathrm{w}$ is chosen less than <\$E1 / 2>: <\$EW ${ }^{\sim}{ }^{\sim}$ roman NTP.FILTER $>$ for filter dispersion and $\left\langle \$ E^{\sim}{ }^{\sim}=\sim\right.$ roman NTP.SELECT $>$ for select dispersion. The (absolute) dispersion <\$Eepsilon sub sigma> and <\$Eepsilon sub $\mathrm{xi}>$ as used in the NTP algorithms are defined relative to the 0th entry $<$ Eepsilon sub $0>$.

There are two procedures described in the following, the clock-filter procedure, which is used to select the best offset samples from a given clock, and the clock-selection procedure, which is used to select the best clock among a hierarchical set of clocks. 
The clock-filter procedure is executed upon arrival of an NTP message or other event that results in new data samples. It takes arguments of the form (<\$Etheta, delta, epsilon>), where <\$Etheta> is a sample clock offset measurement and <\$Edelta> and <\$Eepsilon> are the associated roundtrip delay and dispersion. It determines the filtered clock offset (peer.offset), roundtrip delay (peer.delay) and dispersion (peer.dispersion). It also updates the dispersion of samples already recorded and saves the current time (peer.update).

The basis of the clock-filter procedure is the filter shift register (peer.filter), which consists of NTP.SHIFT stages, each stage containing a 3-tuple <\$E[ theta sub $i$, delta sub $i$, epsilon sub $i$ ] $>$, with indices numbered from zero on the left. The filter is initialized with the value <\$E[0, 0, roman NTP.MAXDISPERSE]> in all stages by the clear procedure. New data samples are shifted into the filter at the left end, causing first NULLs then old samples to fall off the right end. The packet procedure provides samples of the form (<\$Etheta, delta , epsilon>) as new updates arrive, while the transmit procedure provides samples of the form <\$E[0, 0, roman NTP.MAXDISPERSE]> when two poll intervals elapse without a fresh update. While the same symbols (<\$Etheta, delta, epsilon>) are used here for the arguments, clockfilter contents and peer variables, the meaning will be clear from context. The following pseudo-code describes this procedure.

begin clock-filter procedure (<\$Etheta, 〜elta, , epsilon>)

The dispersion <\$Eepsilon sub i> for all valid samples in the filter register must be updated to account for the skew-error accumulation since the last update. These samples are also inserted on a temporary list with entry format <\$E[distance, index]>. The samples in the register are shifted right one stage, with the overflow sample discarded and the new sample inserted at the leftmost stage. The temporary list is then sorted by increasing distance. If no samples remain in the list, the procedure exits without updating the peer variables.

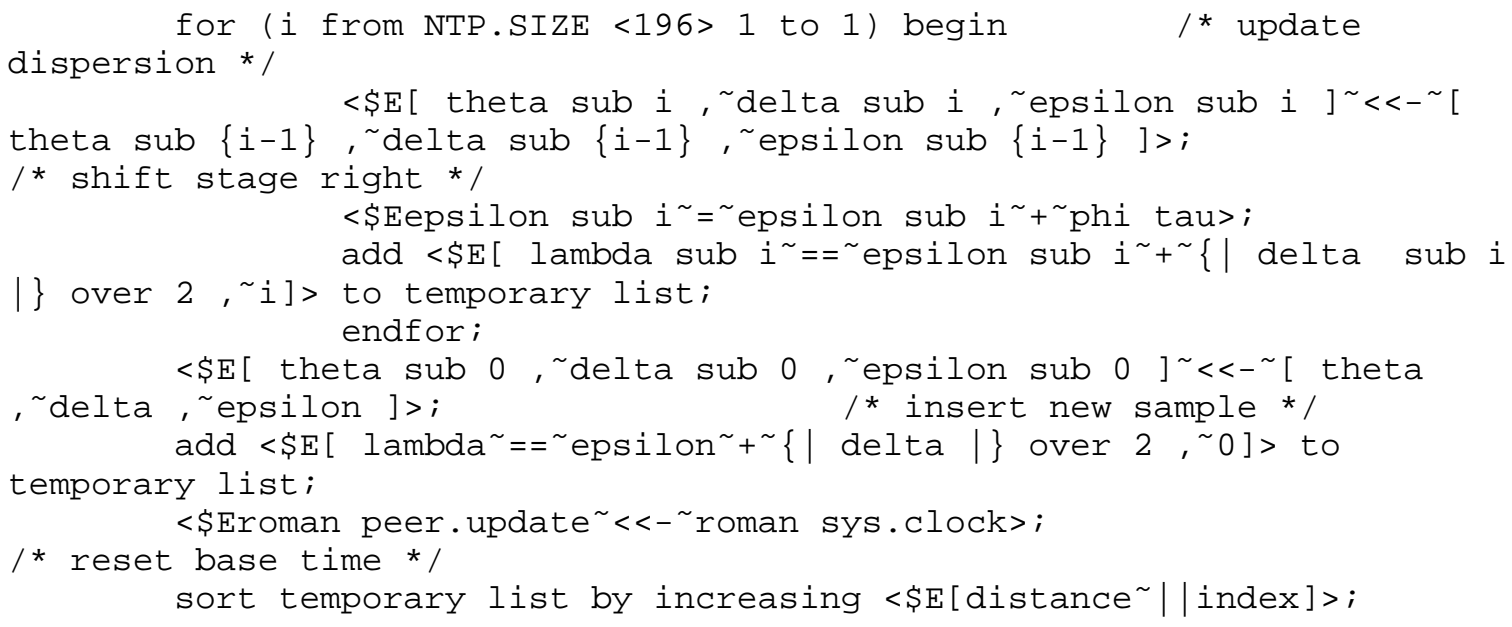

where <\$E[distance || index] > represents the concatenation of the distance and index fields and distance is the high-order field. The filter dispersion <\$Eepsilon sub sigma> is computed and included in the peer dispersion. Note that for this purpose the temporary list is already sorted.

$<$ Eepsilon sub sigma $\sim<-\sim 0>$;

for ( $i$ from NTP.SHIFT<196>1 to 0 ) /* compute

filter dispersion */

if (<\$Eroman peer.dispersion sub index[i] $>>=\sim$ roman

NTP.MAXDISPERSE > or

$$
<\$ E \mid \text { theta sub } i^{\sim}-\sim \text { theta sub } 0 \mid \sim>>\text { roman }
$$

NTP . MAXDISPERSE > ) 
The peer offset <\$Etheta sub 0>, delay <\$Edelta sub $0>$ and dispersion $<$ Eepsilon sub $0>$ are chosen as the values corresponding to the minimumdistance sample; in other words, the sample corresponding to the first entry on the temporary list, here represented as the 0th subscript.

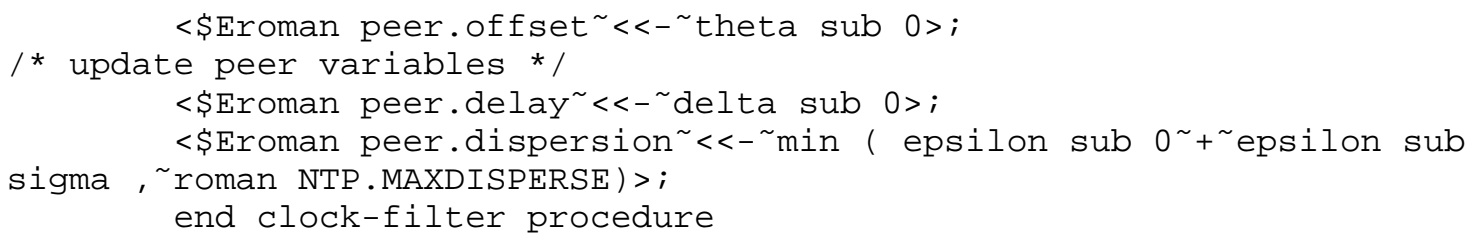

The peer.offset and peer.delay variables represent the clock offset and roundtrip delay of the local clock relative to the peer clock. Both of these are precision quantities and can usually be averaged over long intervals in order to improve accuracy and stability without bias accumulation (see Appendix H). The peer.dispersion variable represents the maximum error due to measurement error, skew-error accumulation and sample variance. All three variables are used in the clock-selection and clock-combining procedures to select the peer clock(s) used for synchronization and to maximize the accuracy and stability of the indications.

Clock-Selection Procedure

The clock-selection procedure uses the peer variables <\$ETHETA>, <\$EDELTA>, <\$EEPSILON> and <\$Etau> and is called when these variables change or when the reachability status changes. It consists of two algorithms, the intersection algorithm and the clustering algorithm. The intersection algorithm constructs a list of candidate peers eligible to become the synchronization source, computes a confidence interval for each and casts out falsetickers using a technique adapted from Marzullo and Owicki [MAR85]. The clustering algorithm sorts the list of surviving candidates in order of stratum and synchronization distance and repeatedly casts out outlyers on the basis of select dispersion until only the most accurate, precise and stable survivors are left. A bit is set for each survivor to indicate the outcome of the selection process. The system variable sys.peer is set as a pointer to the most likely survivor, if there is one, or to the NULL value if not.

Intersection Algorithm

$$
\text { begin clock-selection procedure }
$$

Each peer is examined in turn and added to an endpoint list only if it passes several sanity checks designed to avoid loops and use of exceptionally noisy data. If no peers survive the sanity checks, the procedure exits without finding a synchronization source. For each of $\mathrm{m}$ survivors three entries of the form <\$E[endpoint, type] $>$ are added to the endpoint list: <\$E[ THETA $\left.\left.{ }^{\sim} \sim \mathrm{LAMBDA}, \sim-\sim 1\right]\right\rangle,\langle \$ E[\mathrm{THETA}, \sim 0]\rangle$ and $<$ \$E [ THETA ${ }^{\sim}{ }^{\sim}$ LAMBDA, $\left.\sim 1\right]>$. There will be <\$E3 m> entries on the list, which is then sorted by increasing endpoint.

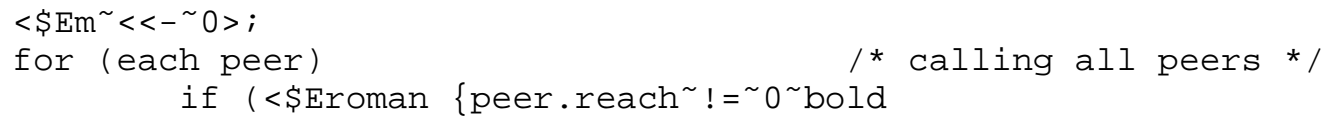


add <\$E $\left[\mathrm{THETA}^{\sim}+{ }^{\sim} \mathrm{LAMBDA}, \sim 1\right]>$ to endpoint list;

$<\$ E m^{\sim}<<-\sim \mathrm{m}^{\sim}+\sim 1>$;

$<$ B $>$ endif

endfor

if $\left(<\$ E m^{\sim}=\sim 0>\right)$ begin /* skedaddle if

no candidates $\star /$

$<$ Eroman sys.peer ${ }^{\sim}<<-\sim$ roman NULL $>$;

$<$ Eroman sys.stratum ${ }^{\sim}<-^{\sim} 0^{\sim}$ (undefined) $>$;

exit;

endif

sort endpoint list by increasing endpoint||type;

The following algorithm is adapted from DTS [DEC89] and is designed to produce the largest single intersection containing only truechimers. The algorithm begins by initializing a value $f$ and counters $i$ and $c$ to zero. Then, starting from the lowest endpoint of the sorted endpoint list, for each entry <\$E[endpoint, type]> the value of type is subtracted from the counter $i$, which is the number of intersections. If type is zero, increment the value of $c$, which is the number of falsetickers (see Appendix H). If $\left\langle \$ E i^{\sim}>>=^{\sim} \mathrm{m}^{\sim}{ }^{\sim}\right.$ f $\rangle$ for some entry, endpoint of that entry becomes the lower endpoint of the intersection; otherwise, $f$ is increased by one and the above procedure is repeated. Without resetting $f$ or $c$, a similar procedure is used to find the upper endpoint, except that the value of type is added to the counter.. If after both endpoints have been determined $\left\langle \$ E_{C}{ }^{\sim}<=^{\sim} f>\right.$, the procedure continues having found $<\$ E m^{\sim}-\sim$ f $>$ truechimers; otherwise, $f$ is increased by one and the entire procedure is repeated.

for (f from 0 to $\left\langle \$ E^{\sim}\right\rangle>=\sim m$ over $2>$ ) begin /* calling all truechimers */ $<\$ \mathrm{EC}^{\sim}<<-\sim 0>$; $<\mathrm{Ei} \sim<-\sim 0>$;

low endpoint */

for (each [endpoint, type] from lowest) begin /* find

$<$ Ei $\sim<-\sim i \sim \sim$ type $>$;

$<$ ElOW ${ }^{\sim}<<-\sim$ endpoint $>$;

if $\left(\left\langle \$ \mathrm{Ei} \sim>>\sim \mathrm{m}^{\sim} \sim \sim \mathrm{f}\right\rangle\right)$ break;

if $\left(<\right.$ Etype $\left.{ }^{\sim} \sim 0>\right) \quad<$ EC $\sim<<{ }^{\sim} \mathrm{C}^{\sim}+\sim 1>$;

endfor

$<\$ E i^{\sim}<<-\sim 0>$;

high endpoint */

for (each [endpoint, type] from highest) begin /* find

$<$ Ei $\sim<-\sim i \sim+$ type $>$;

$<$ Ehigh $\sim<-\sim$ endpoint $>$;

if $\left(\left\langle \$ \mathrm{Ei} \sim>>=\sim \mathrm{m}^{\sim} \sim \sim \mathrm{f}>\right)\right.$ break;

if $\left(<\$ E_{\text {type }} \sim{ }^{\sim} 0>\right) \quad<\$ \mathrm{EC}^{\sim}<<-\sim \mathrm{C}^{\sim}+\sim 1>$;

endfor:

if $\left(<\$ E C^{\sim}<<=\sim\right.$ f $\left.>\right)$ break; /* continue

until all falsetickers found */

endfor;

if $\left(\left\langle\right.\right.$ Elow ${ }^{\sim}>>^{\sim}$ high $>$ ) begin /* quit

if no intersection found */

$<$ SEroman sys.peer ${ }^{\sim}<<-\sim$ roman NULL $>$;

exit;

endif;

Note that processing continues past this point only if there are more than <\$Em over 2> intersections. However, it is possible, but not highly likely, that there may be fewer than <\$Em over 2> truechimers remaining in the intersection.

Clustering Algorithm

In the original DTS algorithm the clock-selection procedure exits at this point with the presumed correct time set midway in the computed intersection <\$E[low, $\sim$ high] $>$. However, this can lead to a considerable 
loss in accuracy and stability, since the individual peer statistics are lost. Therefore, in NTP the candidates that survived the preceding steps are processed further. The candidate list is rebuilt with entries of the form <\$E[distance, index]>, where distance is computed from the (scaled) peer stratum and synchronization distance <\$ELAMBDA>. The scaling factor provides a mechanism to weight the combination of stratum and distance. Ordinarily, the stratum will dominate, unless one or more of the survivors has an exceptionally high distance. The list is then sorted by increasing distance.

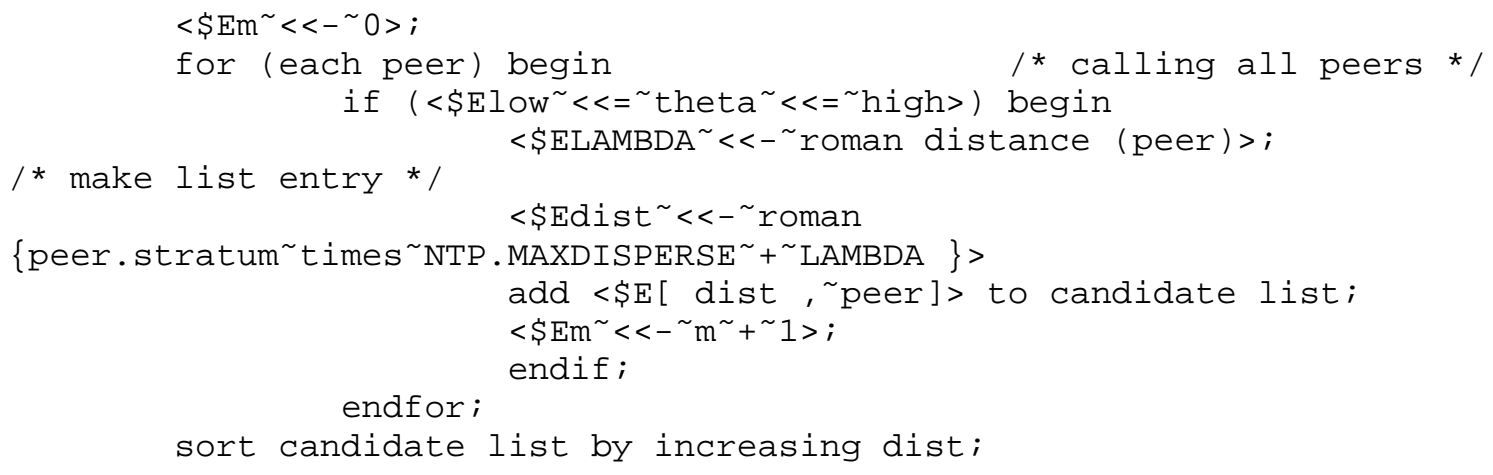

The next steps are designed to cast out outlyers which exhibit significant dispersions relative to the other members of the candidate list while minimizing wander, especially on high-speed LANs with many time servers. Wander causes needless network overhead, since the poll interval is clamped at sys.poll as each new peer is selected for synchronization and only slowly increases when the peer is no longer selected. It has been the practical experience that the number of candidates surviving to this point can become quite large and can result in significant processor cycles without materially enhancing stability and accuracy. Accordingly, the candidate list is truncated at NTP.MAXCLOCK entries.

Note <\$Eepsilon sub $\{x i \mathrm{i}\}>$ is the select (sample) dispersion relative to the ith peer represented on the candidate list, which can be calculated in a manner similar to the filter dispersion described previously. The <\$EEPSILON sub $j>$ is the dispersion of the jth peer represented on the list and includes components due to measurement error, skew-error accumulation and filter dispersion. If the maximum $<$ Eepsilon sub $\{\mathrm{xi} i\}>$ is greater than the minimum <\$EEPSILON sub $j>$ and the number of survivors is greater than NTP.MINCLOCK, the ith peer is discarded from the list and the procedure is repeated. If the current synchronization source is one of the survivors and there is no other survivor of lower stratum, then the procedure exits without doing anything further. Otherwise, the synchronization source is set to the first survivor on the candidate list. In the following $i, j, k, l$ are peer indices, with $\mathrm{k}$ the index of the current synchronization source (NULL if none) and $l$ the index of the first survivor on the candidate list.

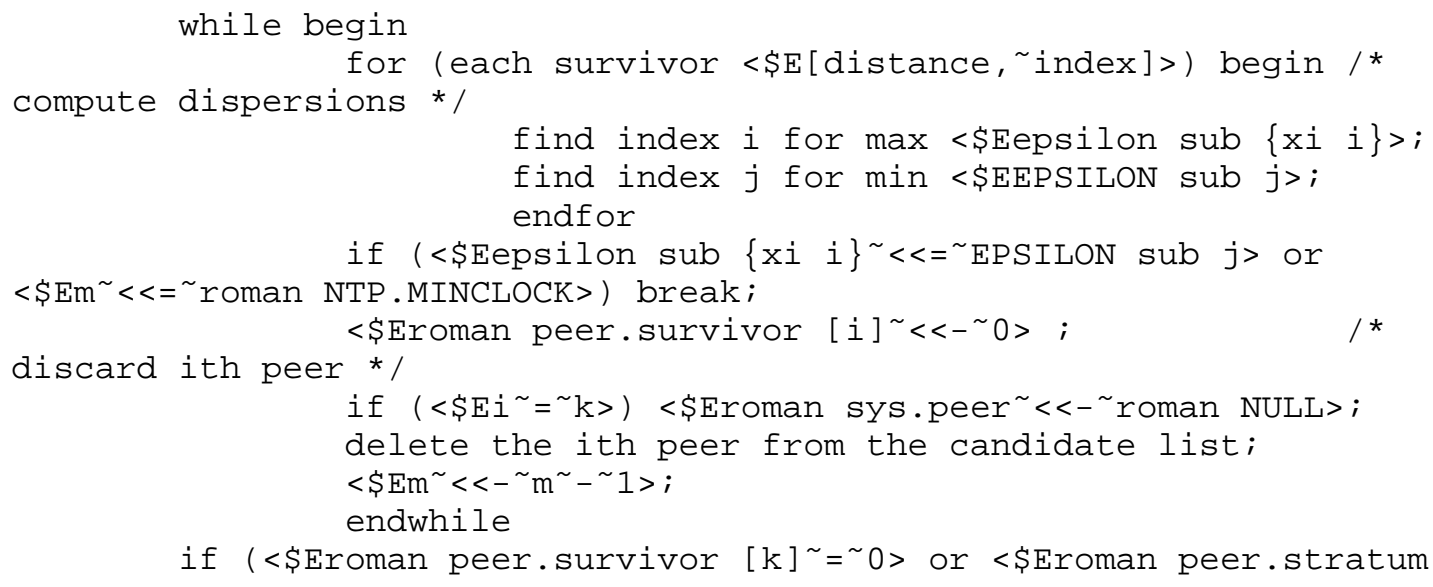




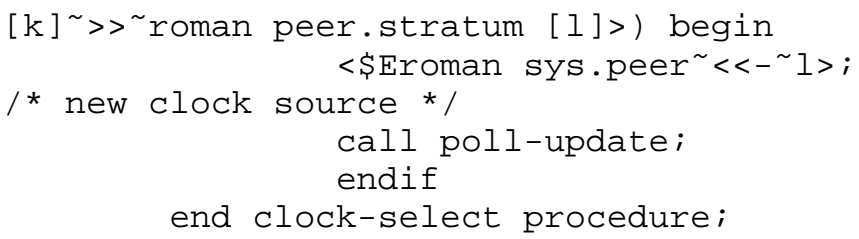

The algorithm is designed to favor those peers near the head of the candidate list, which are at the lowest stratum and distance and presumably can provide the most accurate and stable time. With proper selection of weight factor $v$ (also called NTP.SELECT), entries will be trimmed from the tail of the list, unless a few outlyers disagree significantly with respect to the remaining entries, in which case the outlyers are discarded first. The termination condition is designed to avoid needless switching between synchronization sources when not statistically justified, yet maintain a bias toward the low-stratum, low-distance peers.

\section{Local Clocks}

In order to implement a precise and accurate local clock, the host must be equipped with a hardware clock consisting of an oscillator and interface and capable of the required precision and stability. A logical clock is then constructed using these components plus software components that adjust the apparent time and frequency in response to periodic updates computed by NTP or some other time-synchronization protocol such as Hellospeak [MIL83b] or the Unix 4.3bsd TSP [GUS85a]. This section describes the Fuzzball local-clock model and implementation, which includes provisions for precise time and frequency adjustment and can maintain time to within 15 ns and frequency to within $0.3 \mathrm{~ms}$ per day. The model is suitable for use with both compensated and uncompensated quartz oscillators and can be adapted to power-frequency oscillators. A summary of the characteristics of these and other types of oscillators can be found in Appendix E, while a comprehensive mathematical analysis of the NTP local-clock model can be found in Appendix G.

It is important to note that the particular implementation described is only one of possibly many implementations that provide equivalent functionality. However, it is equally important to note that the clock model described in Appendix $G$ and which is the basis of the implementation involves a particular kind of control-feedback loop that is potentially unstable if the design rules are broken. The model and parameter described in Appendix G are designed to provide accurate and stable time under typical operating conditions using conventional hardware and in the face of disruptions in hardware or network connectivity. The parameters have been engineered for reliable operation in a multi-level hierarchical subnet where unstable operation at one level can disrupt possibly many other levels.

Fuzzball Implementation

The Fuzzball local clock consists of a collection of hardware and software registers, together with a set of algorithms, which implement a logical clock that functions as a disciplined oscillator and synchronizes to an external source. Following is a description of its components and manner of operation. Note that all arithmetic is two's complement integer and all shifts $<169\rangle<<<<<170>$ and $<169>>>>><170>$ are arithmetic (sign-fill for right shifts and zero-fill for left shifts).

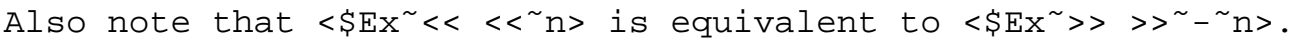

The principal components of the local clock are shown in Figure $3,<\$ \&$ fig3 $>$ in which the fraction points shown are relative to whole milliseconds. The 48-bit clock register and 32-bit Prescaler function as a disciplined oscillator which increments in milliseconds relative to midnight at the fraction point. The 32-bit Clock-Adjust register is used to adjust the oscillator phase in gradual steps to avoid discontinuities 
in the indicated timescale. Its contents are designated $x$ in the following. The 32-bit skew-Compensation register is used to trim the oscillator frequency by adding small phase increments at periodic adjustment intervals and can compensate for frequency errors as much as $.01 \%$ or $<\mathrm{F} 128 \mathrm{M}>\Re<\mathrm{F} 255 \mathrm{D}>100 \mathrm{ppm}$. Its contents are designated $\mathrm{y}$ in the following. The 16-bit Watchdog counter and 32-bit Compliance register are used to determine validity, as well as establish the PLL bandwidth and poll interval (see Appendix G). The contents of the Compliance register are designated $\mathrm{z}$ in the following. The 32-bit PPS-Adjust register is used to hold a precision time adjustment when a source of 1pps pulses is available, while the 8-bit PPS counter is used to verify presence of these pulses. The two-bit Flags register contains the two leap bits described elsewhere (leap).

All registers except the Prescaler register are ordinarily implemented in memory. In typical clock interface designs such as the DEC KWV11-C, the Prescaler register is implemented as a 16-bit buffered counter driven by a quartz-controlled oscillator at some multiple of $1000 \mathrm{~Hz}$. A counter overflow is signalled by an interrupt, which results in an increment of the clock register at the bit corresponding to the overflow. The time of day is determined by reading the Prescaler register, which does not disturb the counting process, and adding its value to that of the clock register with fraction points aligned as shown and with unimplemented low-order bits set to zero. In other interface designs, such as the LSI-11 event-line mechanism, each tick of the clock is signalled by an interrupt at intervals of $16-2 / 3 \mathrm{~ms}$ or 20 ms, depending on interface and mains frequency. When this occurs the appropriate increment in fractional milliseconds is added to the Clock register.

The various parameters used are summarized in Table 6, in which certain parameters have been rescaled from those given in Appendix G due to the units here being in milliseconds.<\$\&tab6> When the system is initialized, all registers and counters are cleared and the leap bits set to 112 (unsynchronized). At adjustment intervals of CLOCK.ADJ seconds CLOCK.ADJ is subtracted from the PPS counter, but only if the previous contents of the PPS counter are greater than zero. Also, CLOCK.ADJ is added to the Watchdog counter, but the latter is clamped not to exceed NTP.MAXAGE divided by CLOCK.ADJ (one full day). In addition, if the Watchdog counter reaches this value, the leap bits are set to 112 (unsynchronized).

In some system configurations a precise source of timing information is available in the form of a train of timing pulses spaced at one-second intervals. Usually, this is in addition to a source of timecode information, such as a radio clock or even NTP itself, to number the seconds, minutes, hours and days. In typical clock interface designs such as the DEC KWV11-C, a special input is provided which can trigger an interrupt as each pulse is received. When this happens the PPS counter is set to CLOCK.PPS and the current time offset is determined in the usual way. Then, the PPS-Adjust register is set to the time offset scaled to milliseconds. Finally, if the PPS-Adjust register is greater than or equal to 500, 1000 is subtracted from its contents. As described below, the PPS-Adjust register and PPS counters can be used in conjunction with an ordinary timecode to produce an extremely accurate local clock.

Gradual Phase Adjustments

Left uncorrected, the local clock runs at the offset and frequency resulting from its last update. An update is produced by an event that results in a valid clock selection. It consists of a signed 48-bit integer in whole milliseconds and fraction, with fraction point to the left of bit 32. If the magnitude is greater than the maximum aperture CLOCK.MAX, a step adjustment is required, in which case proceed as described later. Otherwise, a gradual phase adjustment is performed. Normally, the update is computed by the NTP algorithms described 
previously; however, if the PPS counter is greater than zero, the value of the PPS-Adjust register is used instead. Let u be a 32-bit quantity with bits 0-31 set as bits 16-47 of the update. If some of the low-order bits of the update are unimplemented, they are set as the value of the sign bit. These operations move the fraction point of $u$ to the left of bit 16 and minimize the effects of truncation and roundoff errors. Let b be the number of leading zeros of the absolute value of the Compliance register and let $\mathrm{c}$ be the number of leading zeros of the Watchdog counter, both of which are easily computed by compact loops. Then, set b to

$<\$ E b^{\sim}=\sim \mathrm{b}^{\sim} \sim \sim 16^{\sim}+\sim \operatorname{roman}$ CLOCK. COMP $>$

and clamp it to be not less than zero. This represents the logarithm of the loop time constant. Then, set $c$ to

$<\$ \mathrm{EC}^{\sim}{ }^{\sim} 10^{\sim}-\sim \mathrm{C}>$

and clamp it to be not greater than NTP.MAXPOLL <196> NTP. MINPOLL. This represents the logarithm of the integration interval since the last update. The clamps insure stable operation under typical conditions encountered in the Internet. Then, compute new values for the ClockAdjust and Skew-Compensation registers

$<\$ \mathrm{Ex}^{\sim}=\sim \mathrm{u}^{\sim}>>>>\sim \mathrm{b}>$,

$<\mathrm{EE} \mathrm{Y}^{\sim}=\mathrm{Y}^{\sim}+{ }^{\sim}\left(\mathrm{u}^{\sim}>>>>\sim\left(\mathrm{b}^{\sim}+\sim \mathrm{b}^{\sim}-{ }^{\sim} \mathrm{C}\right)\right)>$.

Finally, compute the exponential average

$<\$ E z^{\sim}=\sim z^{\sim}+\sim\left(u^{\sim}<<<<^{\sim}\left(b^{\sim}+\sim \operatorname{roman} \text { CLOCK.MULT) }{ }^{\sim} z^{\sim}\right)^{\sim}>>>>^{\sim}\right.$ roman

CLOCK.WEIGHT> ,

where the left shift realigns the fraction point for greater precision and ease of computation.

At each adjustment interval the final clock correction consisting of two components is determined. The first (phase) component consists of the quantity

$<\$ \mathrm{Ex}^{\sim}>>>>^{\sim}$ roman CLOCK.PHASE $>$,

which is then subtracted from the previous contents of the Clock-Adjust register to form the new contents of that register. The second (frequency) component consists of the quantity

$<\$ \mathrm{EY}^{\sim}>>>>$ roman CLOCK.FREQ> .

The sum of the phase and frequency components is the final clock correction, which is then added to the clock register. FInally, the Watchdog counter is set to zero. Operation continues in this way until a new correction is introduced.

The value of $\mathrm{b}$ computed above can be used to update the poll interval system variable (sys.poll). This functions as an adaptive parameter that provides a very valuable feature which reduces the polling overhead, especially if the clock-combining algorithm described in Appendix $F$ is used:

$<$ Eroman sys.poll $\sim<-\sim{ }^{\sim} \mathrm{b}^{\sim}+{ }^{\sim}$ roman NTP.MINPOLL $>$.

Under conditions when update noise is high or the hardware oscillator frequency is changing relatively rapidly due to environmental conditions, the magnitude of the compliance increases. With the parameters specified, this causes the loop bandwidth (reciprocal of time constant) to increase and the poll interval to decrease, eventually to NTP.MINPOLL seconds. When noise is low and the hardware oscillator very stable, the compliance decreases, which causes the loop bandwidth to decrease and the poll interval to increase, eventually to NTP.MAXPOLL 
seconds.

The parameters in Table 6 have been selected so that, under good conditions with updates in the order of a few milliseconds, a precision of a millisecond per day (about .01 ppm or 10-8), can be achieved. Care is required in the implementation to insure monotonicity of the clock register and to preserve the highest precision while minimizing the propagation of roundoff errors. Since all of the multiply/divide operations (except those involved with the 1-pps pulses) computed in real time can be approximated by bitwise-shift operations, it is not necessary to implement a full multiply/divide capability in hardware or software.

In the various implementations of NTP for many Unix-based systems it has been the common experience that the single most important factor affecting local-clock stability is the matching of the phase and frequency coefficients to the particular kernel implementation. It is vital that these coefficients be engineered according to the model values, for otherwise the PLL can fail to track normal oscillator variations and can even become unstable.

Step Phase Adjustments

When the magnitude of a correction exceeds the maximum aperture CLOCK.MAX, the possibility exists that the clock is so far out of synchronization with the reference source that the best action is an immediate and wholesale replacement of clock register contents, rather than in gradual adjustments as described above. However, in cases where the sample variance is extremely high, it is prudent to disbelieve a step change, unless a significant interval has elapsed since the last gradual adjustment. Therefore, if a step change is indicated and the Watchdog counter is less than the preconfigured value CLOCK.MINSTEP, the update is ignored and the local-clock procedure exits. These safeguards are especially useful in those system configurations using a calibrated atomic clock or LORAN-C receiver in conjunction with a separate source of seconds-numbering information, such as a radio clock or NTP peer.

If a step change is indicated the update is added directly to the clock register and the Clock-Adjust register and Watchdog counter both set to zero, but the other registers are left undisturbed. Since a step change invalidates data currently in the clock filters, the leap bits are set to 112 (unsynchronized) and, as described elsewhere, the clear procedure is called to purge the clock filters and state variables for all peers. In practice, the necessity to perform a step change is rare and usually occurs when the local host or reference source is rebooted, for example. This is fortunate, since step changes can result in the local clock apparently running backward, as well as incorrect delay and offset measurements of the synchronization mechanism itself.

Considerable experience with the Internet environment suggests the values of CLOCK.MAX tabulated in Table 6 as appropriate. In practice, these values are exceeded with a single time-server source only under conditions of the most extreme congestion or when multiple failures of nodes or links have occurred. The most common case when the maximum is exceeded is when the time-server source is changed and the time indicated by the new and old sources exceeds the maximum due to systematic errors in the primary reference source or large differences in path delays. It is recommended that implementations include provisions to tailor CLOCK.MAX for specific situations. The amount that CLOCK.MAX can be increased without violating the monotonicity requirement depends on the clock register increment. For an increment of $10 \mathrm{~ms}$, as used in many workstations, the value shown in Table 6 can be increased by a factor of five.

Implementation Issues

The basic NTP robustness model is that a host has no other means to 
verify time other than NTP itself. In some equipment a battery-backed clock/calendar is available for a sanity check. If such a device is available, it should be used only to confirm sanity of the timekeeping system, not as the source of system time. In the common assumption (not always justified) that the clock/calendar is more reliable, but less accurate, than the NTP synchronization subnet, the recommended approach at initialization is to set the clock register as determined from the clock/calendar and the other registers, counters and flags as described above. On subsequent updates if the time offset is greater than a configuration parameter (e.g., 1000 seconds), then the update should be discarded and an error condition reported. Some implementations periodically record the contents of the Skew-Compensation register in stable storage such as a system file or NVRAM and retrieve this value at initialization. This can significantly reduce the time to converge to the nominal stability and accuracy regime.

Conversion from NTP format to the common date and time formats used by application programs is simplified if the internal local-clock format uses separate date and time variables. The time variable is designed to roll over at 24 hours, give or take a leap second as determined by the leap-indicator bits, with its overflows (underflows) incrementing (decrementing) the date variable. The date and time variables then indicate the number of days and seconds since some previous reference time, but uncorrected for intervening leap seconds.

On the day prior to the insertion of a leap second the leap bits (sys.leap) are set at the primary servers, presumably by manual means. Subsequently, these bits show up at the local host and are passed to the local-clock procedure. This causes the modulus of the time variable, which is the length of the current day, to be increased or decreased by one second as appropriate. Immediately following insertion the leap bits are reset. Additional discussion on this issue can be found in Appendix E.

Lack of a comprehensive mechanism to administer the leap bits in the primary servers is presently an awkward problem better suited to a comprehensive network-management mechanism yet to be developed. As a practical matter and unless specific provisions have been made otherwise, currently manufactured radio clocks have no provisions for leap seconds, either automatic or manual. Thus, when a leap actually occurs, the radio must resynchronize to the broadcast timecode, which may take from a few minutes to some hours. Unless special provisions are made, a primary server might leap to the new timescale, only to be yanked back to the previous timescale when it next synchronizes to the radio. Subsequently, the server will be yanked forward again when the radio itself resynchronizes to the broadcast timecode.

This problem can not be reliably avoided using any selection algorithm, since there will always exist an interval of at least a couple of minutes and possibly as much as some hours when some or all radios will be out of synchronization with the broadcast timecode and only after the majority of them have resynchronized will the subnet settle down. The CLOCK.MINSTEP delay is designed to cope with this problem by forcing a minimum interval since the last gradual adjustment was made before allowing a step change to occur. Therefore, until the radio resynchronizes, it will continue on the old timescale, which is one second off the local clock after the leap and outside the maximum aperture CLOCK.MAX permitted for gradual phase adjustments. When the radio eventually resynchronizes, it will almost certainly come up within the aperture and again become the reference source. Thus, even in the unlikely case when the local clock incorrectly leaps, the server will go no longer than CLOCK.MINSTEP seconds before resynchronizing.

\section{Acknowledgments}

Many people contributed to the contents of this document, which was thoroughly debated by electronic mail and debugged using two different 
prototype implementations for the Unix 4.3bsd operating system, one written by Louis Mamakos and Michael Petry of the University of Maryland and the other by Dennis Ferguson of the University of Toronto. Another implementation for the Fuzzball operating system [MIL88b] was written by the author. Many individuals to numerous to mention meticulously tested the several beta-test prototype versions and ruthlessly smoked out the bugs, both in the code and the specification. Especially useful were comments from Dennis Ferguson and Bill Sommerfeld, as well as discussions with Joe Comuzi and others at Digital Equipment Corporation.

References

[ABA89]

Abate, et al. AT\&T's new approach to the synchronization of telecommunication networks. IEEE Communications Magazine (April 1989), $35-45$.

\section{[ALL74a]}

Allan, D.W., J.H. Shoaf and D. Halford. Statistics of time and frequency data analysis. In: Blair, B.E. (Ed.). Time and Frequency Theory and Fundamentals. National Bureau of Standards Monograph 140, U.S. Department of Commerce, 1974, 151-204.

[ALL 7 4b]

Allan, D.W., J.E. Gray and H.E. Machlan. The National Bureau of Standards atomic time scale: generation, stability, accuracy and accessibility. In: Blair, B.E. (Ed.). Time and Frequency Theory and Fundamentals. National Bureau of Standards Monograph 140, U.S. Department of Commerce, 1974, 205-231.

[BEL86]

Bell Communications Research. Digital Synchronization Network Plan. Technical Advisory TA-NPL-000436, 1 November 1986.

[BER87]

Bertsekas, D., and R. Gallager. Data Networks. Prentice-Hall, Englewood Cliffs, NJ, 1987 .

[BLA74]

Blair, B.E. Time and frequency dissemination: an overview of principles and techniques. In: Blair, B.E. (Ed.). Time and Frequency Theory and Fundamentals. National Bureau of Standards Monograph 140, U.S. Department of Commerce, 1974, 233-314.

[BRA80]

Braun, W.B. Short term frequency effects in networks of coupled oscillators. IEEE Trans. Communications COM-28, 8 (August 1980), 12691275.

[COL88]

Cole, R., and C. Foxcroft. An experiment in clock synchronisation. The Computer Journal 31, 6 (1988), 496-502.

[DAR81a ]

Defense Advanced Research Projects Agency. Internet Protocol. DARPA Network Working Group Report RFC-791, USC Information Sciences Institute, september 1981. 
[DAR81b]

Defense Advanced Research Projects Agency. Internet Control Message Protocol. DARPA Network Working Group Report RFC-792, USC Information Sciences Institute, September 1981.

[DEC89]

Digital Time Service Functional Specification Version T.1.0.5. Digital Equipment Corporation, 1989.

[DER90 ]

Dershowitz, N., and E.M. Reingold. Calendrical Calculations. Software Practice and Experience 20, 9 (September 1990), 899-928.

[FRA82 ]

Frank, R.L. History of LORAN-C. Navigation 29, 1 (Spring 1982).

[GUS84 ]

Gusella, R., and S. Zatti. TEMPO - A network time controller for a distributed Berkeley UNIX system. IEEE Distributed Processing Technical Committee Newsletter 6, NoSI-2 (June 1984), 7-15. Also in: Proc. Summer USENIX Conference (June 1984, Salt Lake City) .

[GUS85a]

Gusella, R., and S. Zatti. The Berkeley UNIX 4.3BSD time synchronization protocol: protocol specification. Technical Report UCB/CSD 85/250, University of California, Berkeley, June 1985.

[GUS85b]

Gusella, R., and S. Zatti. An election algorithm for a distributed clock synchronization program. Technical Report UCB/CSD 86/275, University of California, Berkeley, December 1985.

[HAL8 4 ]

Halpern, J.Y., B. Simons, R. Strong and D. Dolly. Fault-tolerant clock synchronization. Proc. Third Annual ACM Symposium on Principles of Distributed Computing (August 1984), 89-102.

[JOR85]

Jordan, E.C. (Ed). Reference Data for Engineers, Seventh Edition. H.W. Sams \& Co., New York, 1985.

[KOP 87]

Kopetz, H., and W. Ochsenreiter. Clock synchronization in distributed real-time systems. IEEE Trans. Computers C-36, 8 (August 1987), 933-939.

[LAM78 ]

Lamport, L., Time, clocks and the ordering of events in a distributed system. Comm. ACM 21, 7 (July 1978), 558-565.

[LAM85]

Lamport, L., and P.M. Melliar-Smith. Synchronizing clocks in the presence of faults. J. ACM 32, 1 (January 1985), 52-78. 
Lindsay, W.C., and A.V. Kantak. Network synchronization of random

signals. IEEE Trans. Communications COM-28, 8 (August 1980), 1260-1266.

[LUN84 ]

Lundelius, J., and N.A. Lynch. A new fault-tolerant algorithm for clock synchronization. Proc. Third Annual ACM Symposium on Principles of Distributed Computing (August 1984), 75-88.

[MAR85]

Marzullo, K., and S. Owicki. Maintaining the time in a distributed system. ACM Operating Systems Review 19, 3 (July 1985), 44-54.

[MIL81a]

Mills, D.L. Time Synchronization in DCNET Hosts. DARPA Internet Project Report IEN-173, COMSAT Laboratories, February 1981.

[MIL81b]

Mills, D.L. DCNET Internet Clock Service. DARPA Network Working Group Report RFC-778, COMSAT Laboratories, April 1981.

[MIL83a]

Mills, D.L. Internet Delay Experiments. DARPA Network Working Group Report RFC-889, M/A-COM Linkabit, December 1983.

[MIL83b]

Mills, D.L. DCN local-network protocols. DARPA Network Working Group Report RFC-891, M/A-COM Linkabit, December 1983.

[MIL85a]

Mills, D.L. Algorithms for synchronizing network clocks. DARPA Network Working Group Report RFC-956, M/A-COM Linkabit, September 1985.

[MIL85b]

Mills, D.L. Experiments in network clock synchronization. DARPA Network Working Group Report RFC-957, M/A-COM Linkabit, September 1985.

[MIL85c]

Mills, D.L. Network Time Protocol (NTP). DARPA Network Working Group Report RFC-958, M/A-COM Linkabit, September 1985.

[MIL88a ]

Mills, D.L. Network Time Protocol (version 1) - specification and implementation. DARPA Network Working Group Report RFC-1059, University of Delaware, July 1988.

[MIL88b]

Mills, D.L. The Fuzzball. Proc. ACM SIGCOMM 88 Symposium (Palo Alto, CA, August 1988), 115-122.

[MIL89]

Mills, D.L. Network Time Protocol (version 2) - specification and implementation. DARPA Network Working Group Report RFC-1119, University of Delaware, September 1989. 
[MIL90]

Mills, D.L. Measured performance of the Network Time Protocol in the Internet system. ACM Computer Communication Review 20, 1 (January 1990), $65-75$.

[MIL91a]

Mills, D.L. Internet time synchronization: the Network Time Protocol. IEEE Trans. Communications 39, 10 (October 1991), 1482-1493.

[MIL91b]

Mills, D.L. On the chronology and metrology of computer network timescales and their application to the Network Time Protocol. ACM Computer Communications Review 21, 5 (October 1991), 8-17.

[MIT80]

Mitra, D. Network synchronization: analysis of a hybrid of master-slave and mutual synchronization. IEEE Trans. Communications COM-28, 8 (August $1980), 1245-1259$.

[NBS77]

Data Encryption Standard. Federal Information Processing Standards Publication 46. National Bureau of Standards, U.S. Department of Commerce, 1977 .

[NBS79]

Time and Frequency Dissemination Services. NBS Special Publication 432, U.S. Department of Commerce, 1979 .

[NBS 80 ]

DES Modes of Operation. Federal Information Processing Standards Publication 81. National Bureau of Standards, U.S. Department of Commerce, December 1980 .

$[\mathrm{POS} 80]$

Postel, J. User Datagram Protocol. DARPA Network Working Group Report RFC-768, USC Information Sciences Institute, August 1980.

[POS83a]

Postel, J. Daytime protocol. DARPA Network Working Group Report RFC-867, USC Information Sciences Institute, May 1983.

[POS83b]

Postel, J. Time protocol. DARPA Network Working Group Report RFC-868, USC Information Sciences Institute, May 1983.

[RIC88 ]

Rickert, N.W. Non Byzantine clock synchronization - a programming experiment. ACM Operating Systems Review 22, 1 (January 1988), 73-78.

[SCH86]

Schneider, F.B. A paradigm for reliable clock synchronization. Department of Computer Science Technical Report TR 86-735, Cornell University, February 1986. 
Smith, J. Modern Communications Circuits. McGraw-Hill, New York, NY, 1986.

[SRI87]

Srikanth, T.K., and S. Toueg. Optimal clock synchronization. J. ACM 34, 3 (July 1987), 626-645.

[STE88]

Steiner, J.G., C. Neuman, and J.I. Schiller. Kerberos: an authentication service for open network systems. Proc. Winter USENIX Conference (February 1988).

[SU81]

Su, Z. A specification of the Internet protocol (IP) timestamp option. DARPA Network Working Group Report RFC-781. SRI International, May 1981.

[TRI86]

Tripathi, S.K., and S.H. Chang. ETempo: a clock synchronization algorithm for hierarchical LANs - implementation and measurements. Systems Research Center Technical Report TR-86-48, University of Maryland, 1986.

[VAN8 4 ]

Van Dierendonck, A.J., and W.C. Melton. Applications of time transfer using NAVSTAR GPS. In: Global Positioning System, Papers Published in Navigation, Vol. II, Institute of Navigation, Washington, DC, 1984.

[VAS78]

Vass, E.R. OMEGA navigation system: present status and plans 1977-1980. Navigation 25, 1 (Spring 1978).

Appendix A. NTP Data Format - Version 3

The format of the NTP Message data area, which immediately follows the UDP header, is shown in Figure 4<\$\&fig4>. Following is a description of its fields.

Leap Indicator (LI): This is a two-bit code warning of an impending leap second to be inserted/deleted in the last minute of the current day, with bit 0 and bit 1 , respectively, coded as follows:

QZ_TBL_BEG $=$ COLUMNS (2), DIMENSION (IN), COLWIDTHS $(E 1$, E8), WIDTH $(5.0000)$, ABOVE (.0830), BELOW (.0830), HGUTtER(.0560), $\operatorname{KEEP}(\mathrm{OFF}), \operatorname{ALIGN}(\mathrm{CT})$

QZ_TBL_BODY $=$ TABLE TEXT, TABLE TEXT

00 , no warning

01, last minute has 61 seconds

10, last minute has 59 seconds)

11, alarm condition (clock not synchronized)

QZ_TBL_END =

Version Number (VN): This is a three-bit integer indicating the NTP version number, currently three (3).

Mode: This is a three-bit integer indicating the mode, with values 
defined as follows:

QZ_TBL_BEG $=$ COLUMNS (2), DIMENSION (IN), COLWIDTHS (E1,E8), WIDTH(5.0000), ABOVE (.0830), BELOW(.0830), $\operatorname{HGUTTER}(.0560), \operatorname{KEEP}(\mathrm{OFF}), \operatorname{ALIGN}(\mathrm{CT})$

QZ_TBL_BODY $=$ TABLE TEXT, TABLE TEXT

0 , reserved

1, symmetric active

2, symmetric passive

3, client

4, server

5, broadcast

6, reserved for NTP control message (see Appendix B)

7, reserved for private use

Q Z_TBL_END =

Stratum: This is a eight-bit integer indicating the stratum level of the local clock, with values defined as follows:

QZ_TBL_BEG $=$ COLUMNS (2), DIMENSION (IN), $\operatorname{COLWIDTHS}(E 1$, E 8), WIDTH $(5.0000)$, ABOVE (.0830), BELOW (.0830), $\operatorname{HGUTTER}(.0560), \operatorname{KEEP}(\mathrm{OFF}), \operatorname{ALIGN}(\mathrm{CT})$

QZ_TBL_BODY $=$ TABLE TEXT, TABLE TEXT

0 , unspecified

1, primary reference (e.g., , radio clock)

2-255, secondary reference (via NTP)

Q Z_TBL_END =

The values that can appear in this field range from zero to NTP.INFIN inclusive.

Poll Interval: This is an eight-bit signed integer indicating the maximum interval between successive messages, in seconds to the nearest power of two. The values that can appear in this field range from NTP.MINPOLL to NTP.MAXPOLL inclusive.

Precision: This is an eight-bit signed integer indicating the precision of the local clock, in seconds to the nearest power of two.

Root Delay: This is a 32-bit signed fixed-point number indicating the total roundtrip delay to the primary reference source, in seconds with fraction point between bits 15 and 16. Note that this variable can take on both positive and negative values, depending on clock precision and skew.

Root Dispersion: This is a 32-bit signed fixed-point number indicating the maximum error relative to the primary reference source, in seconds with fraction point between bits 15 and 16. Only positive values greater than zero are possible.

Reference Clock Identifier: This is a 32-bit code identifying the particular reference clock. In the case of stratum 0 (unspecified) or stratum 1 (primary reference), this is a four-octet, left-justified, zero-padded ASCII string. While not enumerated as part of the NTP specification, the following are suggested ASCII identifiers: 
QZ_TBL_BEG $=$ COLUMNS (3), DIMENSION (IN), $\operatorname{COLWIDTHS}(\mathrm{E} 2, \mathrm{E} 2, \mathrm{E} 5)$,

WIDTH (4.6700), $\operatorname{ABOVE}(.1670), \operatorname{BELOW}(.0830), \operatorname{HGUTTER}(.3330)$,

BOX (Z_SINGLE), $\operatorname{KEEP}(\mathrm{ON}), \operatorname{ALIGN}(\mathrm{CT}), \mathrm{L} 1(\mathrm{R} 1 \mathrm{C} 0 \ldots \mathrm{R} 1 \mathrm{C} 3)$

@Z_TBL_BODY = TABLE HEADER, TABLE HEADER, TABLE HEADER

Stratum, Code, Meaning

QZ_TBL_BODY $=$ TABLE TEXT, TABLE TEXT, TABLE TEXT

0 , DCN, DCN routing protocol

0, NIST, NIST public modem

0, TSP, TSP time protocol

0, DTS, Digital Time Service

1, ATOM, Atomic clock (calibrated)

1, VLF, VLF radio (OMEGA, , etc.)

1, callsign, Generic radio

1, LORC, LORAN-C radionavigation

1, GOES, GOES UHF environment satellite

QZ_TBL_BODY = TABLE HEADER, TABLE HEADER, TABLE HEADER

1, GPS, GPS UHF satellite positioning

QZZTBL_END =

In the case of stratum 2 and greater (secondary reference) this is the four-octet Internet address of the primary reference host.

Reference Timestamp: This is the local time at which the local clock was last set or corrected, in 64-bit timestamp format.

Originate Timestamp: This is the local time at which the request departed the client host for the service host, in 64-bit timestamp format.

Receive Timestamp: This is the local time at which the request arrived at the service host, in 64-bit timestamp format.

Transmit Timestamp: This is the local time at which the reply departed the service host for the client host, in 64-bit timestamp format.

Authenticator (optional): When the NTP authentication mechanism is implemented, this contains the authenticator information defined in Appendix C.

Appendix B. NTP Control Messages

In a comprehensive network-management environment, facilities are presumed available to perform routine NTP control and monitoring functions, such as setting the leap-indicator bits at the primary servers, adjusting the various system parameters and monitoring regular operations. Ordinarily, these functions can be implemented using a network-management protocol such as SNMP and suitable extensions to the MIB database. However, in those cases where such facilities are not available, these functions can be implemented using special NTP control messages described herein. These messages are intended for use only in systems where no other management facilities are available or appropriate, such as in dedicated-function bus peripherals. Support for 
these messages is not required in order to conform to this specification.

The NTP Control Message has the value 6 specified in the mode field of the first octet of the NTP header and is formatted as shown below. The format of the data field is specific to each command or response; however, in most cases the format is designed to be constructed and viewed by humans and so is coded in free-form ASCII. This facilitates the specification and implementation of simple management tools in the absence of fully evolved network-management facilities. As in ordinary NTP messages, the authenticator field follows the data field. If the authenticator is used the data field is zero-padded to a 32-bit boundary, but the padding bits are not considered part of the data field and are not included in the field count.

IP hosts are not required to reassemble datagrams larger than 576 octets; however, some commands or responses may involve more data than will fit into a single datagram. Accordingly, a simple reassembly feature is included in which each octet of the message data is numbered starting with zero. As each fragment is transmitted the number of its first octet is inserted in the offset field and the number of octets is inserted in the count field. The more-data (M) bit is set in all fragments except the last.

Most control functions involve sending a command and receiving a response, perhaps involving several fragments. The sender chooses a distinct, nonzero sequence number and sets the status field and $R$ and $E$ bits to zero. The responder interprets the opcode and additional information in the data field, updates the status field, sets the $R$ bit to one and returns the three 32-bit words of the header along with additional information in the data field. In case of invalid message format or contents the responder inserts a code in the status field, sets the $R$ and $E$ bits to one and, optionally, inserts a diagnostic message in the data field.

Some commands read or write system variables and peer variables for an association identified in the command. Others read or write variables associated with a radio clock or other device directly connected to a source of primary synchronization information. To identify which type of variable and association a 16-bit association identifier is used. System variables are indicated by the identifier zero. As each association is mobilized a unique, nonzero identifier is created for it. These identifiers are used in a cyclic fashion, so that the chance of using an old identifier which matches a newly created association is remote. A management entity can request a list of current identifiers and subsequently use them to read and write variables for each association. An attempt to use an expired identifier results in an exception response, following which the list can be requested again.

Some exception events, such as when a peer becomes reachable or unreachable, occur spontaneously and are not necessarily associated with a command. An implementation may elect to save the event information for later retrieval or to send an asynchronous response (called a trap) or both. In case of a trap the IP address and port number is determined by a previous command and the sequence field is set as described below. Current status and summary information for the latest exception event is returned in all normal responses. Bits in the status field indicate whether an exception has occurred since the last response and whether more than one exception has occurred.

Commands need not necessarily be sent by an NTP peer, so ordinary access-control procedures may not apply; however, the optional mask/match mechanism suggested elsewhere in this document provides the capability to control access by mode number, so this could be used to limit access for control messages (mode 6) to selected address ranges. 
The format of the NTP Control Message header, which immediately follows the UDP header, is shown in Figure 5<\$\&fig5>. Following is a description of its fields. Bit positions marked as zero are reserved and should always be transmitted as zero.

Version Number (VN): This is a three-bit integer indicating the NTP version number, currently three (3).

Mode: This is a three-bit integer indicating the mode. It must have the value 6, indicating an NTP control message.

Response Bit (R): Set to zero for commands, one for responses.

Error Bit (E): Set to zero for normal response, one for error response.

More Bit (M): Set to zero for last fragment, one for all others.

Operation Code (Op): This is a five-bit integer specifying the command function. Values currently defined include the following:

QZ_TBL_BEG $=$ COLUMNS (2), DIMENSION (IN), COLWIDTHS $(E 1$, E8), WIDTH $(5.0000)$, ABOVE (.0830), BELOW(.0830), HGUTTER(.0560), $\operatorname{KEEP}(\mathrm{OFF}), \operatorname{ALIGN}(\mathrm{CT})$

QZ_TBL_BODY $=$ TABLE TEXT, TABLE TEXT

0 , reserved

1, read status command/response

2, read variables command/response

3, write variables command/response

4, read clock variables command/response

5, write clock variables command/response

6, set trap address/port command/response

7, trap response

$8-31$, reserved

Q Z_TBL_END $=$

Sequence: This is a 16-bit integer indicating the sequence number of the command or response.

Status: This is a 16-bit code indicating the current status of the system, peer or clock, with values coded as described in following sections.

Association ID: This is a 16-bit integer identifying a valid association.

Offset: This is a 16-bit integer indicating the offset, in octets, of the first octet in the data area.

Count: This is a 16-bit integer indicating the length of the data field, in octets.

Data: This contains the message data for the command or response. The maximum number of data octets is 468 .

Authenticator (optional): When the NTP authentication mechanism is implemented, this contains the authenticator information defined in Appendix C. 
Status words indicate the present status of the system, associations and clock. They are designed to be interpreted by network-monitoring programs and are in one of four 16-bit formats shown in Figure 6<\$\&fig6> and described in this section. System and peer status words are associated with responses for all commands except the read clock variables, write clock variables and set trap address/port commands. The association identifier zero specifies the system status word, while a nonzero identifier specifies a particular peer association. The status word returned in response to read clock variables and write clock variables commands indicates the state of the clock hardware and decoding software. A special error status word is used to report malformed command fields or invalid values.

System Status Word

The system status word appears in the status field of the response to a read status or read variables command with a zero association identifier. The format of the system status word is as follows:

Leap Indicator (LI): This is a two-bit code warning of an impending leap second to be inserted/deleted in the last minute of the current day, with bit 0 and bit 1 , respectively, coded as follows:

QZ_TBL_BEG $=$ COLUMNS (2), DIMENSION (IN), COLWIDTHS $(E 1$, E8), WIDTH $(5.0000)$, ABOVE (.0830), BELOW (.0830), $\operatorname{HGUTTER}(.0560), \operatorname{KEEP}(\mathrm{OFF}), \operatorname{ALIGN}(\mathrm{CT})$

QZ_TBL_BODY $=$ TABLE TEXT, TABLE TEXT

00 , no warning

01, last minute has 61 seconds

10, last minute has 59 seconds)

11, alarm condition (clock not synchronized)

Q Z_TBL_END =

Clock Source: This is a six-bit integer indicating the current synchronization source, with values coded as follows:

QZ_TBL_BEG $=$ COLUMNS (2), DIMENSION (IN), COLWIDTHS (E1,E8), WIDTH $(5.0000)$, ABOVE (.0830), BELOW (.0830), $\operatorname{HGUTTER}(.0560), \operatorname{KEEP}(\mathrm{OFF}), \operatorname{ALIGN}(\mathrm{CT})$

QZ_TBL_BODY $=$ TABLE TEXT, TABLE TEXT

0, unspecified or unknown

1, Calibrated atomic clock (e.g., , HP 5061)

2, VLF (band 4) or LF (band 5) radio (e.g., OMEGA, , WWVB)

3, $\mathrm{HF}$ (band 7) radio (e.g., , CHU, $\mathrm{MSF}$, WWV/H)

4, UHF (band 9) satellite (e.g., , GOES, , GPS)

5, local net (e.g., $\mathrm{DCN}, \mathrm{TSP}, \mathrm{DTS})$

6, UDP/NTP

7, UDP / TIME

8, eyeball-and-wristwatch

9, telephone modem (e.g., , NIST) 
$10-63$, reserved

QZ_TBL_END =

System Event Counter: This is a four-bit integer indicating the number of system exception events occurring since the last time the system status word was returned in a response or included in a trap message. The counter is cleared when returned in the status field of a response and freezes when it reaches the value 15.

System Event Code: This is a four-bit integer identifying the latest system exception event, with new values overwriting previous values, and coded as follows:

QZ_TBL_BEG $=$ COLUMNS (2), DIMENSION (IN), COLWIDTHS $(E 1$, E8), WIDTH $(5.0000)$, ABOVE (.0830), BELOW (.0830), HGUTTER(.0560), $\operatorname{KEEP}(\mathrm{OFF}), \operatorname{ALIGN}(\mathrm{CT})$

QZ_TBL_BODY $=$ TABLE TEXT, TABLE TEXT

0 , unspecified

1, system restart

2, system or hardware fault

3, system new status word (leap bits or synchronization change)

4, system new synchronization source or stratum (sys.peer or sys.stratum change)

5, system clock reset (offset correction exceeds CLOCK.MAX)

6, system invalid time or date (see NTP specification)

7, system clock exception (see system clock status word)

$8-15$, reserved

Q Z_TBL_END =

Peer Status Word

A peer status word is returned in the status field of a response to a read status, read variables or write variables command and appears also in the list of association identifiers and status words returned by a read status command with a zero association identifier. The format of a peer status word is as follows:

Peer Status: This is a five-bit code indicating the status of the peer determined by the packet procedure, with bits assigned as follows:

QZ_TBL_BEG $=$ COLUMNS (2), DIMENSION (IN), COLWIDTHS (E1,E8), WIDTH $(5.0000)$, ABOVE (.0830), BELOW (.0830), HGUTtER(.0560), $\operatorname{KEEP}(\mathrm{OFF}), \operatorname{ALIGN}(\mathrm{CT})$

QZ_TBL_BODY $=$ TABLE TEXT, TABLE TEXT

0 , configured (peer.config)

1 , authentication enabled (peer.authenable)

2, authentication okay (peer.authentic)

3, reachability okay (peer. reach <F128M $>0 ̈<$ F255D>0)

4, reserved

QZ_TBL_END = 
Peer Selection (Sel): This is a three-bit integer indicating the status of the peer determined by the clock-selection procedure, with values coded as follows:

QZ_TBL_BEG $=$ COLUMNS (2), DIMENSION (IN), COLWIDTHS $(E 1$, E8), WIDTH $(5.0000)$, ABOVE (.0830), BELOW (.0830), HGUTtER(.0560), $\operatorname{KEEP}($ OFF), ALIGN(CT)

QZ_TBL_BODY $=$ TABLE TEXT, TABLE TEXT

0 , rejected

1, passed sanity checks (tests 1 through 8 in Section 3.4.3)

2, passed correctness checks (intersection algorithm in Section 4.2.1)

3, passed candidate checks (if limit check implemented)

4, passed outlyer checks (clustering algorithm in section 4.2.2)

5, current synchronization source; max distance exceeded (if limit check implemented)

6, current synchronization source; max distance okay

7, reserved

Q Z_TBL_END =

Peer Event Counter: This is a four-bit integer indicating the number of peer exception events that occurred since the last time the peer status word was returned in a response or included in a trap message. The counter is cleared when returned in the status field of a response and freezes when it reaches the value 15 .

Peer Event Code: This is a four-bit integer identifying the latest peer exception event, with new values overwriting previous values, and coded as follows:

QZ_TBL_BEG $=$ COLUMNS (2), DIMENSION (IN), COLWIDTHS (E1,E8), WIDTH $(5.0000)$, ABOVE (.0830), BELOW (.0830), HGUTtER(.0560), $\operatorname{KEEP}(\mathrm{OFF}), \operatorname{ALIGN}(\mathrm{CT})$

QZ_TBL_BODY $=$ TABLE TEXT, TABLE TEXT

0 , unspecified

1, peer IP error

2, peer authentication failure (peer.authentic bit was one now zero)

3, peer unreachable (peer.reach was nonzero now zero)

4, peer reachable (peer.reach was zero now nonzero)

5, peer clock exception (see peer clock status word)

6-15, reserved

a Z_TBL_END $=$

Clock Status Word

There are two ways a reference clock can be attached to a NTP service host, as an dedicated device managed by the operating system and as a synthetic peer managed by NTP. As in the read status command, the association identifier is used to identify which one, zero for the system clock and nonzero for a peer clock. Only one system clock is supported by the protocol, although many peer clocks can be supported. A 
system or peer clock status word appears in the status field of the response to a read clock variables or write clock variables command. This word can be considered an extension of the system status word or the peer status word as appropriate. The format of the clock status word is as follows:

Clock status: This is an eight-bit integer indicating the current clock status, with values coded as follows:

@Z_TBL_BEG $=$ COLUMNS (2), DIMENSION (IN), COLWIDTHS $(E 1$, E8), WIDTH $(5.0000)$, ABOVE (.0830), BELOW (.0830), $\operatorname{HGUTTER}(.0560), \operatorname{KEEP}(\mathrm{OFF}), \operatorname{ALIGN}(\mathrm{CT})$

QZ_TBL_BODY $=$ TABLE TEXT, TABLE TEXT

0, clock operating within nominals

1, reply timeout

2, bad reply format

3, hardware or software fault

4, propagation failure

5, bad date format or value

6 , bad time format or value

7-255, reserved

Q Z_TBL_END =

Clock Event Code: This is an eight-bit integer identifying the latest clock exception event, with new values overwriting previous values. When a change to any nonzero value occurs in the radio status field, the radio status field is copied to the clock event code field and a system or peer clock exception event is declared as appropriate.

Error Status Word

An error status word is returned in the status field of an error response as the result of invalid message format or contents. Its presence is indicated when the $\mathrm{E}$ (error) bit is set along with the response (R) bit in the response. It consists of an eight-bit integer coded as follows:

@Z_TBL_BEG $=$ COLUMNS (2), DIMENSION (IN), COLWIDTHS $(E 1$, E8), WIDTH $(5.0000)$, ABOVE (.0830), BELOW (.0830), $\operatorname{HGUTTER}(.0560), \operatorname{KEEP}(\mathrm{OFF}), \operatorname{ALIGN}(\mathrm{CT})$

QZ_TBL_BODY $=$ TABLE TEXT, TABLE TEXT

0 , unspecified

1, authentication failure

2, invalid message length or format

3, invalid opcode

4, unknown association identifier

5, unknown variable name

6, invalid variable value

7, administratively prohibited

$8-255$, reserved 
Commands

Commands consist of the header and optional data field shown in Figure 6. When present, the data field contains a list of identifiers or assignments in the form

$<<$ identifier $>>$ [ =<<value $>>$ ], $<<$ identifier $>>[=<<$ value $>>], \ldots$

where <<identifier>> is the ASCII name of a system or peer variable specified in Table 2 or Table 3 and <<value>> is expressed as a decimal, hexadecimal or string constant in the syntax of the $\mathrm{C}$ programming language. Where no ambiguity exists, the <169>sys. $<170>$ or $<169>$ peer. $<170>$ prefixes shown in Table 2 or Table 4 can be suppressed. Whitespace (ASCII nonprinting format effectors) can be added to improve readability for simple monitoring programs that do not reformat the data field. Internet addresses are represented as four octets in the form [n.n.n.n], where $\mathrm{n}$ is in decimal notation and the brackets are optional. Timestamps, including reference, originate, receive and transmit values, as well as the logical clock, are represented in units of seconds and fractions, preferably in hexadecimal notation, while delay, offset, dispersion and distance values are represented in units of milliseconds and fractions, preferably in decimal notation. All other values are represented as-is, preferably in decimal notation.

Implementations may define variables other than those listed in Table 2 or Table 3. Called extramural variables, these are distinguished by the inclusion of some character type other than alphanumeric or $\langle 169\rangle$. $\langle 170\rangle$ in the name. For those commands that return a list of assignments in the response data field, if the command data field is empty, it is expected that all available variables defined in Table 3 or Table 4 of the NTP specification will be included in the response. For the read commands, if the command data field is nonempty, an implementation may choose to process this field to individually select which variables are to be returned.

Commands are interpreted as follows:

Read Status (1): The command data field is empty or contains a list of identifiers separated by commas. The command operates in two ways depending on the value of the association identifier. If this identifier is nonzero, the response includes the peer identifier and status word. Optionally, the response data field may contain other information, such as described in the Read Variables command. If the association identifier is zero, the response includes the system identifier (0) and status word, while the data field contains a list of binary-coded pairs

$<<$ association identifier $>><<$ status word $>>$,

one for each currently defined association. Read Variables (2): The command data field is empty or contains a list of identifiers separated by commas. If the association identifier is nonzero, the response includes the requested peer identifier and status word, while the data field contains a list of peer variables and values as described above. If the association identifier is zero, the data field contains a list of system variables and values. If a peer has been selected as the synchronization source, the response includes the peer identifier and status word; otherwise, the response includes the system identifier (0) and status word.

Write Variables (3): The command data field contains a list of assignments as described above. The variables are updated as indicated. The response is as described for the Read Variables command.

Read Clock Variables (4): The command data field is empty or contains a 
list of identifiers separated by commas. The association identifier selects the system clock variables or peer clock variables in the same way as in the Read Variables command. The response includes the requested clock identifier and status word and the data field contains a list of clock variables and values, including the last timecode message received from the clock.

Write Clock Variables (5): The command data field contains a list of assignments as described above. The clock variables are updated as indicated. The response is as described for the Read Clock Variables command.

Set Trap Address/Port (6): The command association identifier, status and data fields are ignored. The address and port number for subsequent trap messages are taken from the source address and port of the control message itself. The initial trap counter for trap response messages is taken from the sequence field of the command. The response association identifier, status and data fields are not significant. Implementations should include sanity timeouts which prevent trap transmissions if the monitoring program does not renew this information after a lengthy interval.

Trap Response (7): This message is sent when a system, peer or clock exception event occurs. The opcode field is 7 and the $\mathrm{R}$ bit is set. The trap counter is incremented by one for each trap sent and the sequence field set to that value. The trap message is sent using the IP address and port fields established by the set trap address/port command. If a system trap the association identifier field is set to zero and the status field contains the system status word. If a peer trap the association identifier field is set to that peer and the status field contains the peer status word. Optional ASCII-coded information can be included in the data field.

\section{Appendix C. Authentication Issues}

NTP robustness requirements are similar to those of other multiple-peer distributed protocols used for network routing, management and file access. These include protection from faulty implementations, improper operation and possibly malicious replay attacks with or without data modification. These requirements are especially stringent with distributed protocols, since damage due to failures can propagate quickly throughout the network, devastating archives, routes and monitoring systems and even bring down major portions of the network in the fashion of the classic Internet Worm.

The access-control mechanism suggested in the NTP specification responds to these requirements by limiting access to trusted peers. The various sanity checks resist most replay and spoofing attacks by discarding old duplicates and using the originate timestamp as a one-time pad, since it is unlikely that even a synchronized peer can predict future timestamps with the precision required on the basis of past observations alone. In addition, the protocol environment resists jamming attacks by employing redundant time servers and diverse network paths. Resistance to stochastic disruptions, actual or manufactured, are minimized by careful design of the filtering and selection algorithms.

However, it is possible that a determined intruder can disrupt timekeeping operations between peers by subtle modifications of NTP message data, such as falsifying header fields or certain timestamps. In cases where protection from even these types of attacks is required, a specifically engineered message-authentication mechanism based on cryptographic techniques is necessary. Typical mechanisms involve the use of cryptographic certificates, algorithms and key media, together with secure media databases and key-management protocols. Ongoing research efforts in this area are directed toward developing a standard methodology that can be used with many protocols, including NTP. However, while it may eventually be the case that ubiquitous, widely 
applicable authentication methodology may be adopted by the Internet community and effectively overtake the mechanism described here, it does not appear that specific standards and implementations will happen within the lifetime of this particular version of NTP.

The NTP authentication mechanism described here is intended for interim use until specific standards and implementations operating at the network level or transport level are available. Support for this mechanism is not required in order to conform to the NTP specification itself. The mechanism, which operates at the application level, is designed to protect against unauthorized message-stream modification and misrepresentation of source by insuring that unbroken, authenticated paths exist between a trusted, stratum-one server in a particular synchronization subnet and all other servers in that subnet. It employs a crypto-checksum, computed by the sender and checked by the receiver, together with a set of predistributed algorithms, certificates and cryptographic keys indexed by a key identifier included in the message. However, there are no provisions in NTP itself to distribute or maintain the certificates, algorithms or keys. These quantities may occasionally be changed, which may result in inconsistent key information while rekeying is in progress. The nature of NTP itself is quite tolerant to such disruptions, so no particular provisions are included to deal with them.

The intent of the authentication mechanism is to provide a framework that can be used in conjunction with selected mode combinations to build specific plans to manage clockworking communities and implement policy as necessary. It can be selectively enabled or disabled on a per-peer basis. There is no specific plan proposed to manage the use of such schemes; although several possibilities are immediately obvious. In one scenario a group of time servers peers among themselves using symmetric modes and shares one secret key, say key 1, while another group of servers peers among themselves using symmetric modes and shares another secret key, say key 2. Now, assume by policy it is decided that selected servers in group 1 can provide synchronization to group 2, but not the other way around. The selected servers in group 1 are given key 2, but operated only in server mode, so cannot accept synchronization from group 2; however, group 2 has authenticated access to group-1 servers. Many other scenarios are possible with suitable combinations of modes and keys.

A packet format and crypto-checksum procedure appropriate for NTP is specified in the following sections. The cryptographic information is carried in an authenticator which follows the (unmodified) NTP header fields. The crypto-checksum procedure uses the Data Encryption Standard (DES) [NBS77]; however, only the DES encryption algorithm is used and the decryption algorithm is not necessary. This feature is specifically targeted toward governmental sensitivities on the export of cryptographic technology, since the DES decryption algorithm need not be included in NTP software distributions and thus cannot be extracted and used in other applications to avoid message data disclosure.

\section{NTP Authentication Mechanism}

When it is created and possibly at other times, each association is allocated variables identifying the certificate authority, encryption algorithm, cryptographic key and possibly other data. The specific procedures to allocate and initialize these variables are beyond the scope of this specification, as are the association of the identifiers and keys and the management and distribution of the keys themselves. For example and consistency with the conventions of the NTP specification, a set of appropriate peer and packet variables might include the following:

Authentication Enabled Bit (peer.authenable): This is a bit indicating that the association is to operate in the authenticated mode. For configured peers this bit is determined from the startup environment. 
For non-configured peers, this bit is set to one if an arriving message includes the authenticator and set to zero otherwise.

Authenticated Bit (peer.authentic): This is a bit indicating that the last message received from the peer has been correctly authenticated.

Key Identifier (peer.hostkeyid, peer.peerkeyid, pkt.keyid): This is an integer identifying the cryptographic key used to generate the messageauthentication code. The system variable peer.hostkeyid is used for active associations. The peer.peerkeyid variable is initialized at zero (unspecified) when the association is mobilized. For purposes of authentication an unassigned value is interpreted as zero (unspecified).

Cryptographic Keys (sys.key): This is a set of 64-bit DES keys. Each key is constructed as in the Berkeley Unix distributions, which consists of eight octets, where the seven low-order bits of each octet correspond to the DES bits 1-7 and the high-order bit corresponds to the DES oddparity bit 8 . By convention, the unspecified key 0 (zero), consisting of eight odd-parity zero octets, is used for testing and presumed known throughout the NTP community. The remaining keys are distributed using methods outside the scope of NTP.

Crypto-Checksum (pkt.check): This is a crypto-checksum computed by the encryption procedure.

The authenticator field consists of two subfields, one consisting of the pkt.keyid variable and the other the pkt. check variable computed by the encrypt procedure, which is called by the transmit procedure described in the NTP specification, and by the decrypt procedure, which is called by the receive procedure described in the NTP specification. Its presence is revealed by the fact the total datagram length according to the UDP header is longer than the NTP message length, which includes the header plus the data field, if present. For authentication purposes, the NTP message is zero-padded if necessary to a 64-bit boundary, although the padding bits are not considered part of the NTP message itself. The authenticator format shown in Figure $7<\$ \& f i g 7>$ has 96 bits, including a 32-bit key identifier and 64-bit crypto-checksum, and is aligned on a 32-bit boundary for efficient computation. Additional information required in some implementations, such as certificate authority and encryption algorithm, can be inserted between the (padded) NTP message and the key identifier, as long as the alignment conditions are met. Like the authenticator itself, this information is not included in the crypto-checksum. Use of these data are beyond the scope of this specification. These conventions may be changed in future as the result of other standardization activities.

NTP Authentication Procedures

When authentication is implemented there are two additional procedures added to those described in the NTP specification. One of these (encrypt) constructs the crypto-checksum in transmitted messages, while the other (decrypt) checks this quantity in received messages. The procedures use a variant of the cipher-block chaining method described in [NBS80] as applied to DES. In principal, the procedure is independent of DES and requires only that the encryption algorithm operate on 64-bit blocks. While the NTP authentication mechanism specifies the use of DES, other algorithms could be used by prior arrangement.

Encrypt Procedure

For ordinary NTP messages the encryption procedure operates as follows. If authentication is not enabled, the procedure simply exits. If the association is active (modes 1, 3, 5), the key is determined from the system key identifier. If the association is passive (modes 2, 4) the key is determined from the peer key identifier, if the authentic bit is set, or as the default key (zero) otherwise. These conventions allow further protection against replay attacks and keying errors, as well as facilitate testing and migration to new versions. The crypto-checksum is 
calculated using the 64-bit NTP header and data words, but not the authenticator or padding bits.

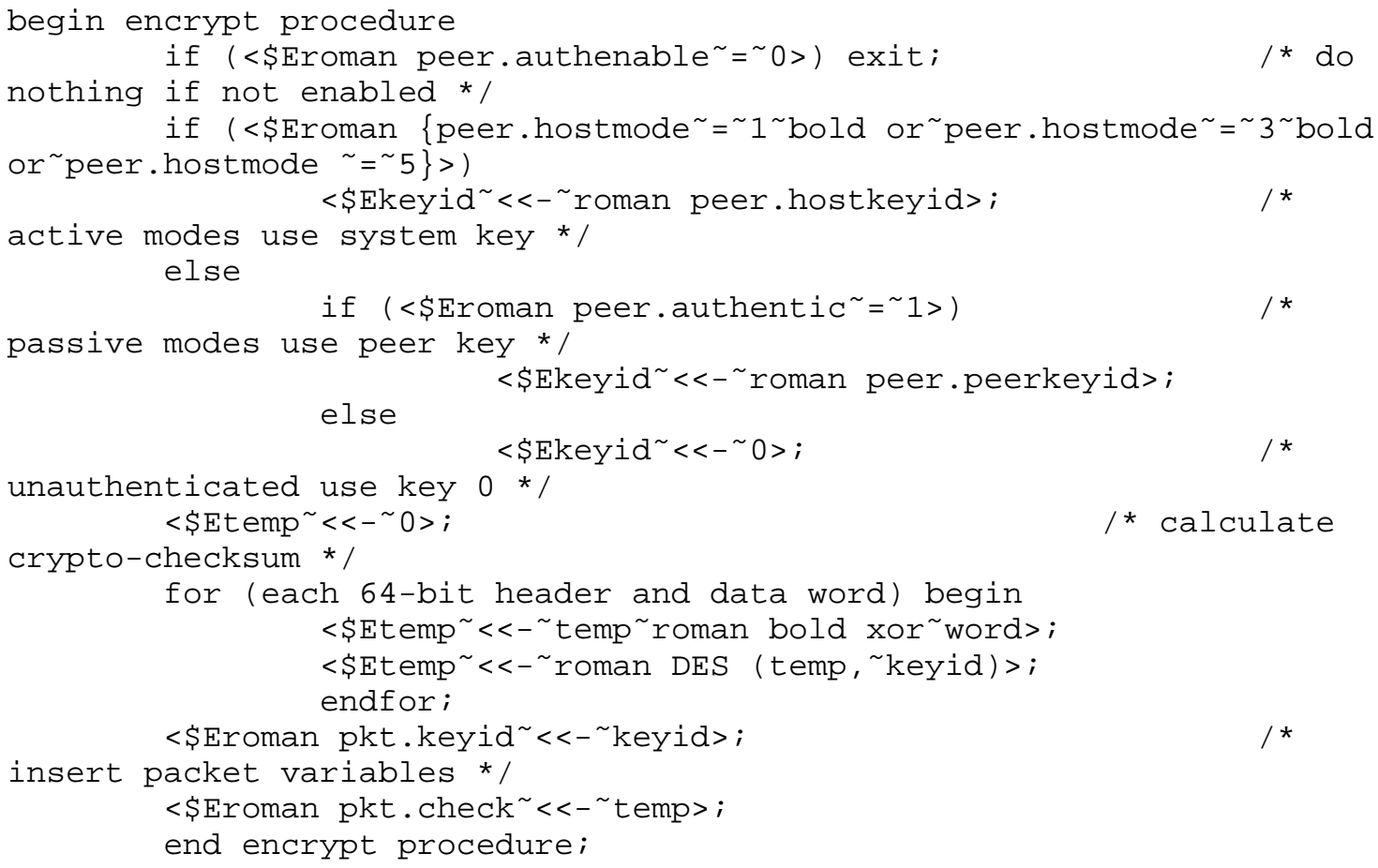


procedure and the response message includes the authenticator field as computed by the encrypt procedure. If the received authenticator is correct, the key for the response is the same as in the command; otherwise, the default key (zero) is used. Commands causing a change to the peer data base, such as the write variables and set trap address/port commands, must be correctly authenticated; however, the remaining commands are normally not authenticated in order to minimize the encryption overhead.

Appendix D. Differences from Previous Versions.

The original NTP, later called NTP Version 0, was described in RFC-958 [MIL85C]. Subsequently, Version 0 was superseded by Version 1 (RFC-1059 [MIL88a]), and Version 2 (RFC-1119 [MIL89]. The Version-2 description was split into two documents, RFC-1119 defining the architecture and specifying the protocol and algorithms, and another [MIL90b] describing the service model, algorithmic analysis and operating experience. In previous versions these two objectives were combined in one document. While the architecture assumed in Version 3 is identical to Version 2, the protocols and algorithms differ in minor ways. Differences between NTP Version 3 and previous versions are described in this Appendix. Due to known bugs in very old implementations, continued support for Version-0 implementations is not recommended. It is recommended that new implementations follow the guidelines below when interoperating with older implementations.

Version 3 neither changes the protocol in any significant way nor obsoletes previous versions or existing implementations. The main motivation for the new version is to refine the analysis and implementation models for new applications at much higher network speeds to the gigabit-per-second regime and to provide for the enhanced stability, accuracy and precision required at such speeds. In particular, the sources of time and frequency errors have been rigorously examined and error bounds established in order to improve performance, provide a model for correctness assertions and indicate timekeeping quality to the user. Version 3 also incorporates two new optional features, (1) an algorithm to combine the offsets of a number of peer time servers in order to enhance accuracy and (2) improved local-clock algorithms which allow the poll intervals on all synchronization paths to be substantially increased in order to reduce network overhead. Following is a summary of previous versions of the protocol together with details of the Version 3 changes.

1 .

Version 1 supports no modes other than symmetric-active and symmetricpassive, which are determined by inspecting the port-number fields of the UDP packet header. The peer mode can be determined explicitly from the packet-mode variable (pkt.mode) if it is nonzero and implicitly from the source port (pkt.peerport) and destination port (pkt.hostport) variables if it is zero. For the case where pkt.mode is zero the mode is determined as follows:

@Z_TBL_BEG $=$ COLUMNS (3), DIMENSION (IN), $\operatorname{WIDTH}(5.0000), \operatorname{ABOVE}(.1670)$, BELOW (.0830), HGUTTER(.3330), BOX(Z_SINGLE), KEEP(ON), ALIGN(CT), L1 (R1C0..R1C3)

QZ_TBL_BODY = TABLE HEADER, TABLE HEADER, TABLE HEADER

pkt.peerport, pkt.hostport, Mode

@Z_TBL_BODY $=$ TABLE TEXT, TABLE TEXT, TABLE TEXT

NTP.PORT, NTP.PORT, symmetric active

NTP.PORT, not NTP.PORT, server

not NTP.PORT, NTP.PORT, client 
not NTP.PORT, not NTP.PORT, not possible

QZ_TBL_END =

Note that it is not possible in this case to distinguish between symmetric active and symmetric passive modes. Use of the pkt.mode and NTP.PORT variables in this way is not recommended and may not be supported in future versions of the protocol. The low-order three bits of the first octet, specified as zero in Version 1, are used for the mode field in Version 2. Version-2 and Version-3 implementations interoperating with Version-1 implementations should operate in a passive mode only and use the value one in the version number (pkt.version) field and zero in the mode (pkt.mode) field in transmitted messages.

2 .

Version 1 does not support the NTP control message described in Appendix B. Certain old versions of the Unix NTP daemon ntpd use the high-order bits of the stratum field (pkt.stratum) for control and monitoring purposes. While these bits are never set during normal Version-1, Version-2 or Version-3 operations, new implementations may use the NTP reserved mode 6 described in Appendix B and/or private reserved mode 7 for special purposes, such as remote control and monitoring, and in such cases the format of the packet following the first octet can be arbitrary. While there is no guarantee that different implementations can interoperate using private reserved mode 7, it is recommended that vanilla ASCII format be used whenever possible.

3.

Version 1 does not support authentication. The key identifiers, cryptographic keys and procedures described in Appendix $C$ are new to Version 2 and continued in Version 3, along with the corresponding variables, procedures and authenticator fields. In the NTP message described in Appendix A and NTP control message described in Appendix B the format and contents of the header fields are independent of the authentication mechanism and the authenticator itself follows the header fields, so that previous versions will ignore the authenticator.

4 .

In Version 1 the total dispersion (pkt.rootdispersion) field of the NTP header was called the estimated drift rate, but not used in the protocol or timekeeping procedures. Implementations of the Version-1 protocol typically set this field to the current value of the skew-compensation register, which is a signed quantity. In a Version 2 implementation apparent large values in this field may affect the order considered in the clock-selection procedure. Version-2 and Version-3 implementations interoperating with older implementations should assume this field is zero, regardless of its actual contents.

5.

Version 2 and Version 3 incorporate several sanity checks designed to avoid disruptions due to unsynchronized, duplicate or bogus timestamp information. The checks in Version 3 are specifically designed to detect lost or duplicate packets and resist invalid timestamps. The leapindicator bits are set to show the unsynchronized state if updates are not received from a reference source for a considerable time or if the reference source has not received updates for a considerable time. Some Version-1 implementations could claim valid synchronization indefinitely following loss of the reference source. 
The clock-selection procedure of Version 2 was considerably refined as the result of accumulated experience with the Version-1 implementation. Additional sanity checks are included for authentication, range bounds and to avoid use of very old data. The candidate list is sorted twice, once to select a relatively few robust candidates from a potentially large population of unruly peers and again to order the resulting list by measurement quality. As in Version 1, The final selection procedure repeatedly casts out outlyers on the basis of weighted dispersion.

7.

The local-clock procedure of Version 2 were considerably improved over Version 1 as the result of analysis, simulation and experience. Checks have been added to warn that the oscillator has gone too long without update from a reference source. The compliance register has been added to improve frequency stability to the order of a millisecond per day. The various parameters were retuned for optimum loop stability using measured data over typical Internet paths and with typical local-clock hardware. In version 3 the phase-lock loop model was further refined to provide an adaptive-bandwidth feature that automatically adjusts for the inherent stabilities of the reference clock and local clock while providing optimum loop stability in each case.

8 .

Problems in the timekeeping calculations of Version 1 with high-speed LANs were found and corrected in Version 2. These were caused by jitter due to small differences in clock rates and different precisions between the peers. Subtle bugs in the Version-1 reachability and polling-rate control were found and corrected. The peer.valid and sys.hold variables were added to avoid instabilities when the reference source changes rapidly due to large dispersive delays under conditions of severe network congestion. The peer.config, peer.authenable and peer.authentic bits were added to control special features and simplify configuration.

9.

In Version 3 The local-clock algorithm has been overhauled to improve stability and accuracy. Appendix G presents a detailed mathematical model and design example which has been refined with the aid of feedback-control analysis and extensive simulation using data collected over ordinary Internet paths. Section 5 of RFC-1119 on the NTP local clock has been completely rewritten to describe the new algorithm. Since the new algorithm can result in message rates far below the old ones, it is highly recommended that they be used in new implementations. Note that this algorithm is not integral to the NTP protocol specification itself and its use does not affect interoperability with previous versions or existing implementations; however, in order to insure overall NTP subnet stability in the Internet, it is essential that the local-clock characteristics of all NTP time servers conform to the analytical models presented previously and in this document.

10 .

In Version 3 a new algorithm to combine the offsets of a number of peer time servers is presented in Appendix F. This algorithm is modelled on those used by national standards laboratories to combine the weighted offsets from a number of standard clocks to construct a synthetic laboratory timescale more accurate than that of any clock separately. It can be used in an NTP implementation to improve accuracy and stability and reduce errors due to asymmetric paths in the Internet. The new algorithm has been simulated using data collected over ordinary Internet paths and, along with the new local-clock algorithm, implemented and tested in the Fuzzball time servers now running in the Internet. Note that this algorithm is not integral to the NTP protocol specification 
itself and its use does not affect interoperability with previous versions or existing implementations.

11.

Several inconsistencies and minor errors in previous versions have been corrected in Version 3. The description of the procedures has been rewritten in pseudo-code augmented by English commentary for clarity and to avoid ambiguity. Appendix I has been added to illustrate C-language implementations of the various filtering and selection algorithms suggested for NTP. Additional information is included in section 5 and in Appendix E, which includes the tutorial material formerly included in Section 2 of RFC-1119, as well as much new material clarifying the interpretation of timescales and leap seconds.

12 .

Minor changes have been made in the Version-3 local-clock algorithms to avoid problems observed when leap seconds are introduced in the UTC timescale and also to support an auxiliary precision oscillator, such as a cesium clock or timing receiver, as a precision timebase. In addition, changes were made to some procedures described in section 3 and in the clock-filter and clock-selection procedures described in section 4. While these changes were made to correct minor bugs found as the result of experience and are recommended for new implementations, they do not affect interoperability with previous versions or existing implementations in other than minor ways (at least until the next leap second).

13.

In Version 3 changes were made to the way delay, offset and dispersion are defined, calculated and processed in order to reliably bound the errors inherent in the time-transfer procedures. In particular, the error accumulations were moved from the delay computation to the dispersion computation and both included in the clock filter and selection procedures. The clock-selection procedure was modified to remove the first of the two sorting/discarding steps and replace with an algorithm first proposed by Marzullo and later incorporated in the Digital Time Service. These changes do not significantly affect the ordinary operation of or compatibility with various versions of NTP, but they do provide the basis for formal statements of correctness as described in Appendix $H$.

Appendix E. The NTP Timescale and its Chronometry

Introduction

Following is an extended discussion on computer network chronometry, which is the precise determination of computer time and frequency relative to international standards and the determination of conventional civil time and date according to the modern calendar. It describes the methods conventionally used to establish civil time and date and the various timescales now in use. In particular, it characterizes the Network Time Protocol (NTP) timescale relative to the Coordinated Universal Time (UTC) timescale, and establishes the precise interpretation of UTC leap seconds in NTP.

In the following discussion the terms time, oscillator, clock, epoch, calendar, date and timescale are used in a technical sense. Strictly speaking, the time of an event is an abstraction which determines the ordering of events in some given frame of reference. An oscillator is a generator capable of precise frequency (relative to the given frame of reference) to a specified tolerance. A clock is an oscillator together with a counter which records the (fractional) number of cycles since being initialized with a given value at a given time. The value of the counter at any given time is called its epoch at that time. In general, 
epoches are not continuous and depend on the precision of the counter.

A calendar is a mapping from epoch in some frame of reference to the times and dates used in everyday life. Since multiple calendars are in use today and sometimes disagree on the dating of the same events in the past, the chronometry of past and present events is an art practiced by historians. One of the goals of this discussion is to provide a standard chronometry for precision dating of present and future events in a global networking community. To synchronize frequency means to adjust the oscillators in the network to run at the same frequency, to synchronize time means to set the clocks so that all agree at a particular epoch with respect to UTC, as provided by international standards, and to synchronize clocks means to synchronize them in both frequency and time.

In order to synchronize clocks, there must be some way to directly or indirectly compare them in time and frequency. The ultimate frame of reference for our world consists of the cosmic oscillators: the Sun, Moon and other galactic orbiters. Since the frequencies of these oscillators are relatively unstable and not known exactly, the ultimate reference standard oscillator has been chosen by international agreement as a synthesis of many observations of an atomic transition of exquisite stability. The epoches of each heavenly and Earthbound oscillator defines a distinctive timescale, not necessarily always continuous, relative to the standard oscillator. Another goal of this presentation is to describe a standard chronometry to rationalize conventional computer time and UTC; in particular, how to handle leap seconds.

Primary Frequency and Time Standards

A primary frequency standard is an oscillator that can maintain extremely precise frequency relative to a physical phenomenon, such as a transition in the orbital states of an electron. Presently available atomic oscillators are based on the transitions of the hydrogen, cesium and rubidium atoms. Table $7<\$ \& t a b 7>$ shows the characteristics for typical oscillators of these types compared with those for various types of quartz-crystal oscillators found in electronic equipment. For reasons of cost and robustness cesium oscillators are used worldwide for national primary frequency standards. On the other hand, local clocks used in computing equipment almost always are designed with uncompensated crystal oscillators.

For the three atomic oscillators listed in Table 7 the drift/aging column shows the maximum offset per day from nominal standard frequency due to systematic mechanical and electrical characteristics. In the case of crystal oscillators this offset is not constant, which results in a gradual change in frequency with time, called aging. Even if a crystal oscillator is temperature compensated by some means, it must be periodically compared to a primary standard in order to maintain the highest accuracy. For all types of oscillators the stability column shows the maximum variation in frequency per day due to circuit noise and environmental factors.

As the telephone networks of the world are evolving rapidly to digital technology, consideration should be given to the methods used for frequency synchronization in digital networks. A network of clocks in which each oscillator is phase-locked to a single frequency standard is called isochronous, while a network in which some oscillators are phaselocked to different master oscillators, but with the master oscillators closely synchronized in frequency (not necessarily phase locked), to a single frequency standard is called plesiochronous. In plesiochronous systems the phase of some oscillators can slip relative to others and cause occasional data errors in synchronous transmission systems.

The industry has agreed on a classification of clock oscillators as a function of minimum accuracy, minimum stability and other factors [ALL74a]. There are three factors which determine the classification: 
stability, jitter and wander. Stability refers to the systematic variation of frequency with time and is synonymous with aging, drift, trends, etc. Jitter (also called timing jitter) refers to short-term variations in frequency with components greater than $10 \mathrm{~Hz}$, while wander refers to long-term variations in frequency with components less than 10 $\mathrm{Hz}$. The classification determines the oscillator stratum (not to be confused with the NTP stratum), with the more accurate oscillators assigned the lower strata and less accurate oscillators the higher strata:

QZ_TBL_BEG $=$ COLUMNS (3), DIMENSION (IN), $\operatorname{COLWIDTHS}(E 1, E 2, E 2)$, WIDTH (5.0000), ABOVE(.1670), BELOW(.0830), $\operatorname{HGUTTER}(.3330)$, BOX (Z_SINGLE), $\operatorname{KEEP}(\mathrm{ON}), \operatorname{ALIGN}(\mathrm{CT}), \mathrm{L} 1(\mathrm{R} 1 \mathrm{C} 0 \ldots \mathrm{R} 1 \mathrm{C} 3)$

@Z_TBL_BODY = TABLE CENTER, TABLE HEADER, TABLE HEADER

Stratum, Min Accuracy (per day), Min Stability (per day)

QZ_TBL_BODY $=$ TABLE CENTER, TABLE TEXT, TABLE TEXT

1, 1 × 10-11, not specified

$2,1.6 \times 10-8,1 \times 10-10$

$3,4.6 \times 10-6,3.7 \times 10-7$

QZ_TBL_BODY = TABLE CENTER, TABLE HEADER, TABLE HEADER

$4,3.2 \times 10-5$, not specified

QZZTBL_END =

The construction, operation and maintenance of stratum-one oscillators is assumed to be consistent with national standards and often includes cesium oscillators or precision crystal oscillators synchronized via LORAN-C to national standards. Stratum-two oscillators represent the stability required for interexchange toll switches such as the AT\&T 4ESS and interexchange digital cross-connect systems, while stratum-three oscillators represent the stability required for exchange switches such as the AT\&T 5ESS and local cross-connect systems. Stratum-four oscillators represent the stability required for digital channel-banks and PBX systems.

Time and Frequency Dissemination

In order that atomic and civil time can be coordinated throughout the world, national administrations operate primary time and frequency standards and coordinate them cooperatively by observing various radio broadcasts and through occasional use of portable atomic clocks. Most seafaring nations of the world operate some sort of broadcast time service for the purpose of calibrating chronographs, which are used in conjunction with ephemeris data to determine navigational position. In many countries the service is primitive and limited to seconds-pips broadcast by marine communication stations at certain hours. For instance, a chronograph error of one second represents a longitudinal position error of about 0.23 nautical mile at the Equator.

The U.S. National Institute of Standards and Technology (NIST - formerly National Bureau of Standards) operates three radio services for the dissemination of primary time and frequency information. One of these uses high-frequency (HF or CCIR band 7) transmissions on frequencies of 2.5, 5, 10, 15 and $20 \mathrm{MHz}$ from Fort Collins, CO (WWV), and Kauai, HI (WWVH). Signal propagation is usually by reflection from the upper ionospheric layers, which vary in height and composition throughout the day and season and result in unpredictable delay variations at the receiver. The timecode is transmitted over a 60-second interval at a data rate of $1 \mathrm{bps}$ using a $100-\mathrm{Hz}$ subcarrier on the broadcast signal. 
The timecode information includes UTC time-day information, but does not currently include year or leap-second warning. While these transmissions and those of Canada from Ottawa, Ontario (CHU), and other countries can be received over large areas in the western hemisphere, reliable frequency comparisons can be made only to the order of 10-7 and time accuracies are limited to the order of a millisecond [BLA74]. Radio clocks which operate with these transmissions include the Traconex 1020, which provides accuracies to about ten milliseconds and is priced in the $\$ 1,500$ range.

A second service operated by NIST uses low-frequency (LF or CCIR band 5) transmissions on $60 \mathrm{kHz}$ from Boulder, CO (WWVB), and can be received over the continental U.S. and adjacent coastal areas. Signal propagation is via the lower ionospheric layers, which are relatively stable and have predictable diurnal variations in height. The timecode is transmitted over a 60-second interval at a rate of 1 pps using periodic reductions in carrier power. With appropriate receiving and averaging techniques and corrections for diurnal and seasonal propagation effects, frequency comparisons to within 10-11 are possible and time accuracies of from a few to 50 microseconds can be obtained [BLA74]. Some countries in western Europe operate similar services which use transmissions on 60 $\mathrm{kHz}$ from Rugby, U.K. (MSF), and on $77.5 \mathrm{kHz}$ from Mainflingen, West Germany (DCF77). The timecode information includes UTC time-day-year information and leap-second warning. Radio clocks which operate with these transmissions include the Spectracom 8170 and Kinemetrics/TrueTime 60-DC and LF-DC, which provide accuracies to a millisecond or less and are priced in the $\$ 2,500$ range. However, these receivers do not extract the year information and leap-second warning.

The third service operated by NIST uses ultra-high frequency (UHF or CCIR band 9) transmissions on about $468 \mathrm{MHz}$ from the Geosynchronous Orbit Environmental Satellites (GOES), three of which cover the western hemisphere. The timecode is interleaved with messages used to interrogate remote sensors and consists of 60 4-bit binary-coded decimal words transmitted over an interval of 30 seconds. The timecode information includes UTC time-day-year information and leap-second warning. Radio clocks which operate with these transmissions include the Kinemetrics/TrueTime 468-DC, which provides accuracies to $0.5 \mathrm{~ms}$ and is priced in the $\$ 6,000$ range. However, this receiver does not extract the year information and leap-second warning.

The U.S. Department of Defense is developing the Global Positioning System (GPS) for worldwide precision navigation. This system will eventually provide 24-hour worldwide coverage using a constellation of 24 satellites in 12-hour orbits. For time-transfer applications GPS has a potential accuracy in the order of a few nanoseconds; however, various considerations of defense policy may limit accuracy to hundreds of nanoseconds [VAN84]. The timecode information includes GPS time and UTC correction; however, there appears to be no leap-second warning. Radio clocks which operate with these transmissions include the Kinemetrics/TrueTime GPS-DC, which provides accuracies to $200<\$ E m u>S$ and is priced in the $\$ 12,000$ range. However, since only about half the satellites have been launched, expensive rubidium or quartz oscillators are necessary to preserve accuracy during outages. Also, since this is a single-channel receiver, it must be supplied with geographic coordinates within a degree from an external source before operation begins.

The U.S. Coast Guard, along with agencies of other countries, has operated the LORAN-C [FRA82] radionavigation system for many years. It currently provides time-transfer accuracies of less than a microsecond and eventually may achieve $100 \mathrm{~ns}$ within the ground-wave coverage area of a few hundred kilometers from the transmitter. Beyond the ground wave area signal propagation is via the lower ionospheric layers, which decreases accuracies to the order of 50 us. With the recent addition of the Mid-Continent Chain, the deployment of LORAN-C transmitters now provides complete coverage of the U.S. LORAN-C timing receivers, such as the Austron 2000, are specialized and extremely expensive (up to 
$\$ 20,000)$. They are used primarily to monitor local cesium clocks and are not suited for unattended, automatic operation. While the LORAN-C system provides a highly accurate frequency and time reference within the ground wave area, there is no timecode modulation, so the receiver must be supplied with UTC time to within a few tens of seconds from an external source before operation begins.

The OMEGA [VAS78] radionavigation system operated by the U.S. Navy and other countries consists of eight very-low-frequency (VLF or CCIR band 4 ) transmitters operating on frequencies from 10.2 to $13.1 \mathrm{kHz}$ and providing 24-hour worldwide coverage. With appropriate receiving and averaging techniques and corrections for propagation effects, frequency comparisons and time accuracies are comparable to the LF systems, but with worldwide coverage [BLA74]. Radio clocks which operate with these transmissions include the Kinemetrics/TrueTime OM-DC, which provides accuracies to $1 \mathrm{~ms}$ and is priced in the $\$ 3,500$ range. While the OMEGA system provides a highly accurate frequency reference, there is no timecode modulation, so the receiver must be supplied with geographic coordinates within a degree and UTC time within five seconds from an external source before operation begins. There are several other VLF services intended primarily for worldwide data communications with characteristics similar to OMEGA. These services can be used in a manner similar to OMEGA, but this requires specialized techniques not suited for unattended, automatic operation.

Note that not all transmission formats used by NIST radio broadcast services [NBS79] and no currently available radio clocks include provisions for year information and leap-second warning. This information must be determined from other sources. NTP includes provisions to distribute advance warnings of leap seconds using the leap-indicator bits described in the NTP specification. The protocol is designed so that these bits can be set manually or by the radio timecode at the primary time servers and then automatically distributed throughout the synchronization subnet to all other time servers. Calendar systems

The calendar systems used in the ancient world reflect the agricultural, political and ritual needs characteristic of the societies in which they flourished. Astronomical observations to establish the winter and summer solstices were in use three to four millennia ago. By the 14th century BC the Shang Chinese had established the solar year as 365.25 days and the lunar month as 29.5 days. The lunisolar calendar, in which the ritual month is based on the Moon and the agricultural year on the Sun, was used throughout the ancient Near East (except Egypt) and Greece from the third millennium BC. Early calendars used either thirteen lunar months of 28 days or twelve alternating lunar months of 29 and 30 days and haphazard means to reconcile the 354/364-day lunar year with the 365-day vague solar year.

The ancient Egyptian lunisolar calendar had twelve 30-day lunar months, but was guided by the seasonal appearance of the star sirius (Sothis). In order to reconcile this calendar with the solar year, a civil calendar was invented by adding five intercalary days for a total of 365 days. However, in time it was observed that the civil year was about one-fourth day shorter than the actual solar year and thus would precess relative to it over a 1460-year cycle called the Sothic cycle. Along with the Shang Chinese, the ancient Egyptians had thus established the solar year at 365.25 days, or within about 11 minutes of the present measured value. In 432 BC, about a century after the chinese had done so, the Greek astronomer Meton calculated there were 110 lunar months of 29 days and 125 lunar months of 30 days for a total of 235 lunar months in 6940 solar days, or just over 19 years. The 19-year cycle, called the Metonic cycle, established the lunar month at 29.532 solar days, or within about two minutes of the present measured value.

The Roman republican calendar was based on a lunar year and by 50 BC was eight weeks out of step with the solar year. Julius Caesar invited the 
Alexandrian astronomer Sosigenes to redesign the calendar, which led to the adoption in 46 BC of the Julian calendar. This calendar is based on a year of 365 days with an intercalary day inserted every four years. However, for the first 36 years an intercalary day was mistakenly inserted every three years instead of every four. The result was 12 intercalary days instead of nine, and a series of corrections that was not complete until $8 \mathrm{AD}$.

The seven-day Sumerian week was introduced only in the fourth century $A D$ by Emperor Constantine I. During the Roman era a 15-year census cycle, called the Indiction cycle, was instituted for taxation purposes. The sequence of day-names for consecutive occurrences of a particular day of the year does not recur for 28 years, called the solar cycle. Thus, the least common multiple of the 28-year solar cycle, 19-year Metonic cycle and 15-year Indiction cycle results in a grand 7980-year supercycle called the Julian Era, which began in 4713 BC. A particular combination of the day of the week, day of the year, phase of the Moon and round of the census will recur beginning in $3268 \mathrm{AD}$.

By 1545 the discrepancy in the Julian year relative to the solar year had accumulated to ten days. In 1582, following suggestions by the astronomers Christopher Clavius and Luigi Lilio, Pope Gregory XIII issued a papal bull which decreed, among other things, that the solar year would consist of 365.2422 days. In order to more closely approximate the new value, only those centennial years divisible by 400 would be leap years, while the remaining centennial years would not, making the actual value 365.2425, or within about 26 seconds of the current measured value. Since the beginning of the Common Era and prior to 1990 there were 474 intercalary days inserted in the Julian calendar, but 14 of these were removed in the Gregorian calendar. While the Gregorian calendar is in use throughout most of the world today, some countries did not adopt it until early in the twentieth century. While it remains a fascinating field for time historians, the above narrative provides conclusive evidence that conjugating calendar dates of significant events and assigning NTP timestamps to them is approximate at best. In principle, reliable dating of such events requires only an accurate count of the days relative to some globally alarming event, such as a comet passage or supernova explosion; however, only historically persistent and politically stable societies, such as the ancient Chinese and Egyptian, and especially the classic Maya, possessed the means and will to do so.

The Modified Julian Day System

In order to measure the span of the universe or the decay of the proton, it is necessary to have a standard day-numbering plan. Accordingly, the International Astronomical Union has adopted the use of the standard second and Julian Day Number (JDN) to date cosmological events and related phenomena. The standard day consists of 86,400 standard seconds, where time is expressed as a fraction of the whole day, and the standard year consists of 365.25 standard days.

In the scheme devised in 1583 by the French scholar Joseph Julius Scaliger and named after his father, Julius Caesar Scaliger, JDN 0.0 corresponds to $12 \mathrm{~h}$ (noon) on the first day of the Julian Era, 1 January 4713 BC. The years prior to the Common Era (BC) are reckoned according to the Julian calendar, while the years of the Common Era (AD) are reckoned according to the Gregorian calendar. Since 1 January 1 AD in the Gregorian calendar corresponds to 3 January 1 in the Julian calendar [DER90], JDN 1,721,426.0 corresponds to $12 \mathrm{~h}$ on the first day of the Common Era, 1 January $1 \mathrm{AD}$. The Modified Julian Date (MJD), which is sometimes used to represent dates near our own era in conventional time and with fewer digits, is defined as MJD = JD <196> 2,400,000.5. Following the convention that our century began at $0 \mathrm{~h}$ on 1 January 1900, at which time the tropical year was already $12 \mathrm{~h}$ old, that eclectic instant corresponds to MJD 15,020.0. Thus, the Julian timescale ticks in standard (atomic) 365.25-day centuries and was set to a given value at 
the approximate epoch of a cosmic event which apparently synchronized the entire human community, the origin of the Common Era.

\section{Determination of Frequency}

For many years the most important use of time and frequency information was for worldwide navigation and space science, which depend on astronomical observations of the Sun, Moon and stars [JOR85]. Sidereal time is based on the transit of stars across the celestial meridian of an observer. The mean sidereal day is 23 hours, 56 minutes and 4.09 seconds, but varies about $\angle \mathrm{F} 128 \mathrm{M}>æ<\mathrm{F} 255 \mathrm{D}>30 \mathrm{~ms}$ throughout the year due to polar wandering and orbit variations. Ephemeris time is based on tables with which a standard time interval such as the tropical year one complete revolution of the Earth around the sun - can be determined through observations of the Sun, Moon and planets. In 1958 the standard second was defined as 1/31,556,925.9747 of the tropical year that began this century. On this scale the tropical year is 365.2421987 days and the lunar month - one complete revolution of the Moon around the Earth is 29.53059 days; however, the actual tropical year can be determined only to an accuracy of about $50 \mathrm{~ms}$ and has been increasing by about 5.3 ms per year.

Of the three heavenly oscillators readily apparent to ancient mariners and astronomers - the Earth rotation about its axis, the Earth revolution around the sun and the Moon revolution around the Earth none of the three have the intrinsic stability, relative to modern technology, to serve as a standard reference oscillator. In 1967 the standard second was redefined as <169>9,192,631,770 periods of the radiation corresponding to the transition between the two hyperfine levels of the ground state of the cesium-133 atom.<170> Since 1972 the time and frequency standards of the world have been based on International Atomic Time (TAI), which is defined and maintained using multiple cesium-beam oscillators to an accuracy of a few parts in 1013, or better than a microsecond per day. Note that, while this provides an extraordinarily precise timescale, it does not necessarily agree with conventional solar time and may not in fact even be absolutely uniform, unless subtle atomic conspiracies can be ruled out.

Determination of Time and Leap Seconds

The International Bureau of Weights and Measures (IBWM) uses astronomical observations provided by the U.S. Naval Observatory and other observatories to determine UTC. Starting from apparent mean solar time as observed, the UTO timescale is determined using corrections for Earth orbit and inclination (the Equation of Time, as used by sundials), the UT1 (navigator's) timescale by adding corrections for polar migration and the UT2 timescale by adding corrections for known periodicity variations. While standard frequencies are based on TAI, conventional civil time is based on UT1, which is presently slowing relative to TAI by a fraction of a second per year. When the magnitude of correction approaches 0.7 second, a leap second is inserted or deleted in the TAI timescale on the last day of June or December.

For the most precise coordination and timestamping of events since 1972, it is necessary to know when leap seconds are implemented in UTC and how the seconds are numbered. As specified in CCIR Report 517, which is reproduced in [BLA74], a leap second is inserted following second 23:59:59 on the last day of June or December and becomes second 23:59:60 of that day. A leap second would be deleted by omitting second 23:59:59 on one of these days, although this has never happened. Leap seconds were inserted prior to 1 January 1991 on the occasions listed in Table $8<\$ \&$ tab8 $>$ (courtesy U.S. Naval Observatory). Published IBWM corrections consist not only of leap seconds, which result in step discontinuities relative to TAI, but 100-ms UT1 adjustments called DUT1, which provide increased accuracy for navigation and space science.

Note that the NTP time column actually shows the epoch following the 
last second of the day given in the UTC date and MJD columns (except for the first line), which is the precise epoch of insertion. The offset column shows the cumulative seconds offset between the uncoordinated (Julian) timescale and the UTC timescale; that is, the number of seconds to add to the Julian clock in order to maintain nominal agreement with the UTC clock. Finally, note that the epoch of insertion is relative to the timescale immediately prior to that epoch; e.g., the epoch of the 31 December 90 insertion is determined on the timescale in effect following the 31 December 1990 insertion, which means the actual insertion relative to the Julian clock is fourteen seconds later than the apparent time on the UTC timescale.

The UTC timescale thus ticks in standard (atomic) seconds and was set to the value Oh MJD 41,317.0 at the epoch determined by astronomical observation to be $\mathrm{oh}$ on 1 January 1972 according to the Gregorian calendar; that is, the inaugural tick of the UTC Era. In fact, the inaugural tick which synchronized the cosmic oscillators, Julian clock, UTC clock and Gregorian calendar forevermore was displaced about ten seconds from the civil clock then in use, while the GPS clock is ahead of the UTC clock by six seconds in late 1990. Subsequently, the UTC clock has marched backward relative to the Julian timescale exactly one second on scheduled occasions at monumental epoches embedded in the institutional memory of our civilization. Note in passing that leapsecond adjustments affect the number of seconds per day and thus the number of seconds per year. Apparently, should we choose to worry about it, the UTC clock, Julian clock and various cosmic clocks will inexorably drift apart with time until rationalized by some future papal bull.

The NTP Timescale and Reckoning with UTC The NTP timescale is based on the UTC timescale, but not necessarily always coincident with it. At 0 h on 1 January 1972 (MJD 41,317.0), the first tick of the UTC Era, the NTP clock was set to 2,272,060,800, representing the number of standard seconds since $0 \mathrm{~h}$ on 1 January 1900 (MJD 15,020.0). The insertion of leap seconds in UTC and subsequently into NTP does not affect the UTC or NTP oscillator, only the conversion to conventional civil UTC time. However, since the only institutional memory available to NTP are the UTC timecode broadcast services, the NTP timescale is in effect reset to UTC as each timecode is received. Thus, when a leap second is inserted in UTC and subsequently in NTP, knowledge of all previous leap seconds is lost.

Another way to describe this is to say there are as many NTP timescales as historic leap seconds. In effect, a new timescale is established after each new leap second. Thus, all previous leap seconds, not to mention the apparent origin of the timescale itself, lurch backward one second as each new timescale is established. If a clock synchronized to NTP in 1990 was used to establish the UTC epoch of an event that occurred in early 1972 without correction, the event would appear fifteen seconds late relative to UTC. However, NTP primary time servers resolve the epoch using the broadcast timecode, so that the NTP clock is set to the broadcast value on the current timescale. As a result, for the most precise determination of epoch relative to the historic UTC clock, the user must subtract from the apparent NTP epoch the offsets shown in Table 8 at the relative epoches shown. This is a feature of almost all present day time-distribution mechanisms.

The chronometry involved can be illustrated with the help of Figure 8, which shows the details of seconds numbering just before, during and after the last scheduled leap insertion at 23:59:59 on 31 December 1989. Notice the NTP leap bits are set on the day prior to insertion, as indicated by the $\langle 169\rangle+\langle 170\rangle$ symbols on the figure. Since this makes the day one second longer than usual, the NTP day rollover will not occur until the end of the first occurrence of second 800 . The UTC time conversion routines must notice the apparent time and the leap bits and handle the timescale conversions accordingly. Immediately after the leap insertion both timescales resume ticking the seconds as if the leap had 
never happened. The chronometric correspondence between the UTC and NTP timescales continues, but NTP has forgotten about all past leap insertions. In NTP chronometric determination of UTC time intervals spanning leap seconds will thus be in error, unless the exact times of insertion are known.

It is possible that individual systems may use internal data formats other than the NTP timestamp format, which is represented in seconds to a precision of about 200 picoseconds; however, a persuasive argument exists to use a two-part representation, one part for whole days (MJD or some fixed offset from it) and the other for the seconds (or some scaled value, such as milliseconds). This not only facilitates conversion between NTP and conventional civil time, but makes the insertion of leap seconds much easier. All that is required is to change the modulus of the seconds counter, which on overflow increments the day counter. This design insures that continuity of the timescale is assured, even if outside synchronization is lost before, during or after leap-second insertion. Since timestamp data are unaffected, synchronization is assured, even if timestamp data are in flight at the instant and originated before or at that instant.

Appendix F. The NTP Clock-Combining Algorithm

Introduction

A common problem in synchronization subnets is systematic time-offset errors resulting from asymmetric transmission paths, where the networks or transmission media in one direction are substantially different from the other. The errors can range from microseconds on high-speed ring networks to large fractions of a second on satellite/landline paths. It has been found experimentally that these errors can be considerably reduced by combining the apparent offsets of a number of time servers to produce a more accurate working offset. Following is a description of the combining method used in the NTP implementation for the Fuzzball [MIL88b]. The method is similar to that used by national standards laboratories to determine a synthetic laboratory timescale from an ensemble of cesium clocks [ALL74b]. These procedures are optional and not required in a conforming NTP implementation.

In the following description the stability of a clock is how well it can maintain a constant frequency, the accuracy is how well its frequency and time compare with national standards and the precision is how precisely these quantities can be maintained within a particular timekeeping system. Unless indicated otherwise, The offset of two clocks is the time difference between them, while the skew is the frequency difference (first derivative of offset with time) between them. Real clocks exhibit some variation in skew (second derivative of offset with time), which is called drift.

Determining Time and Frequency

Figure 9<\$\&fig9> shows the overall organization of the NTP time-server model. Timestamps exchanged with possibly many other subnet peers are used to determine individual roundtrip delays and clock offsets relative to each peer as described in the NTP specification. As shown in the figure, the computed delays and offsets are processed by the clock filter to reduce incidental timing noise and the most accurate and reliable subset determined by the clock-selection algorithm. The resulting offsets of this subset are first combined as described below and then processed by the phase-locked loop (PLL). In the PLL the combined effects of the filtering, selection and combining operations is to produce a phase-correction term. This is processed by the loop filter to control the local clock, which functions as a voltage-controlled oscillator (VCO). The VCO furnishes the timing (phase) reference to produce the timestamps used in all calculations. 
The International Standard (SI) definition of time interval is in terms of the standard second: <169>the duration of 9,192,631,770 periods of the radiation corresponding to the transition between the two hyperfine levels of the ground state of the cesium-133 atom.<170> Let u represent the standard unit of time interval so defined and $\left\langle \$ E v^{\sim}=^{\sim} 1\right.$ over $\left.u\right\rangle$ be the standard unit of frequency. The epoch, denoted by $t$, is defined as the reading of a counter that runs at frequency $v$ and began counting at some agreed initial epoch to, which defines the standard or absolute timescale. For the purposes of the following analysis, the epoch of the standard timescale, as well as the time indicated by a clock will be considered continuous. In practice, time is determined relative to a clock constructed from an atomic oscillator and system of counter/dividers, which defines a timescale associated with that particular oscillator. Standard time and frequency are then determined from an ensemble of such timescales and algorithms designed to combine them to produce a composite timescale approximating the standard timescale.

Let $\langle \$ E T(t)>$ be the time displayed by a clock at epoch t relative to the standard timescale:

$<\operatorname{ST}(t) \sim{ }^{\sim} 1 / 2 \mathrm{D}(t \operatorname{sub} 0)\left[t^{\sim}-^{\sim} t \operatorname{sub} 0\right.$ ] $\sup 2^{\sim}+{ }^{\sim} R(t$ sub 0$)\left[t^{\sim}-^{\sim} t\right.$ sub 0 ]$^{\sim}+{ }^{\sim} \mathrm{T}(\mathrm{t}$ sub 0$) \sim+\sim \mathrm{x}(t)>$,

where $\langle$ SED(t sub 0 ) > is the fractional frequency drift per unit time, $<\$ E R(t$ sub 0$)>$ the frequency and $<\$ E T(t$ sub 0 ) $\rangle$ the time at some previous epoch to. In the usual stationary model these quantities can be assumed constant or changing slowly with epoch. The random nature of the clock is characterized by $\langle \$ E x(t)\rangle$, which represents the random noise (jitter) relative to the standard timescale. In the usual analysis the second-order term $\langle \$ E D(t$ sub 0$)\rangle$ is ignored and the noise term $\langle \$ E x(t)\rangle$ modelled as a normal distribution with predictable spectral density or autocorrelation function.

The probability density function of time offset <\$Eroman $\left.\mathrm{p}\left(\mathrm{t}^{\sim}-^{\sim} \mathrm{T}(\mathrm{t})\right)\right\rangle$ usually appears as a bell-shaped curve centered somewhere near zero. The width and general shape of the curve are determined by $\langle \$ E x(t)\rangle$, which depends on the oscillator precision and jitter characteristics, as well as the measurement system and its transmission paths. Beginning at epoch to the offset is set to zero, following which the bell creeps either to the left or right, depending on the value of $\langle \$ E R(t$ sub 0 ) $\rangle$ and accelerates depending on the value of $<\$ E D(t$ sub 0 ) $>$.

Development of a Composite Timescale

Now consider the time offsets of a number of real clocks connected by real networks. A display of the offsets of all clocks relative to the standard timescale will appear as a system of bell-shaped curves slowly precessing relative to each other, but with some further away from nominal zero than others. The bells will normally be scattered over the offset space, more or less close to each other, with some overlapping and some not. The problem is to estimate the true offset relative to the standard timescale from a system of offsets collected routinely between the clocks.

A composite timescale can be determined from a sequence of offsets measured between the $\mathrm{n}$ clocks of an ensemble at nominal intervals $<$ Etau>. Let $<\$ E R$ sub $i(t$ sub 0 ) > be the frequency and <\$ET sub $i$ ( $t$ sub 0 ) $>$ the time of the ith clock at epoch to relative to the standard timescale and let $\langle 169\rangle^{\wedge}\langle 170\rangle$ designate the associated estimates. Then, an estimator for $\mathrm{Ti}$ computed at to for epoch <\$Et sub $0^{\sim}+^{\sim}$ tau $>$ is

$<$ ET hat sub $i\left(t \text { sub } 0^{\sim}+^{\sim} \text { tau }\right)^{\sim}{ }^{\sim}{ }^{\sim} R$ hat sub $i$ (t sub 0 ) tau ${ }^{\sim}+^{\sim} \mathrm{T}$ sub i $(t$ sub 0$)>$, 
offset measurements made between the clocks at epoch <\$Et sub 0 + $^{\sim}$ tau> and let the offset between clock $i$ and clock $j$ at that epoch be <\$ET sub ij ( $t$ sub $0^{\sim}+^{\sim}$ tau ) $>$, defined as

$<$ SET sub ij (t sub $0^{\sim}+^{\sim}$ tau $)^{\sim}==^{\sim} T$ sub $i$ (t sub $0^{\sim}+^{\sim}$ tau $)^{\sim}-^{\sim} T$ sub j ( sub $0^{\sim}+^{\sim}$ tau $)>$.

Note that $\left\langle \$ E T\right.$ sub $i j^{\sim}=\sim-T$ sub $j i>$ and $\left\langle \$ E T\right.$ sub $i i^{\sim}=\sim 0>$. Let $<\$ E w$ sub $i$ ( tau ) > be a previously determined weight factor associated with the ith clock for the nominal interval <\$Etau>. The basis for new estimates at epoch $<\$ E t$ sub $0^{\sim}+^{\sim}$ tau $>$ is

$<$ ET sub j (t sub $0^{\sim}+\sim$ tau ) ${ }^{\sim}{ }^{\sim} \operatorname{sum}$ from $\{i=1\}$ to $\mathrm{n}$ w sub $i$ ( tau ) $\mathrm{T}$ hat sub i (t sub $0^{\sim}+{ }^{\sim}$ tau ) ${ }^{\sim}{ }^{\sim} T$ sub ji (t sub $0^{\sim}+{ }^{\sim}$ tau ) ].>

That is, the apparent time indicated by the jth clock is a weighted average of the estimated time of each clock at epoch <\$Et sub $0 \sim^{\sim}$ tau> plus the time offset measured between the jth clock and that clock at epoch <\$Et sub $0 \sim+^{\sim}$ tau>.

An intuitive grasp of the behavior of this algorithm can be gained with the aid of a few examples. For instance, if <\$Ew sub i ( tau ) > is unity for the ith clock and zero for all others, the apparent time for each of the other clocks is simply the estimated time <\$ET hat sub $i$ ( $t$ sub $0^{\sim}+{ }^{\sim}$ tau $)>$. If $\langle \$ E W$ sub $i($ tau $)>$ is zero for the ith clock, that clock can never affect any other clock and its apparent time is determined entirely from the other clocks. If <\$Ew sub i ( tau ) ${ }^{\sim}{ }^{\sim} / \mathrm{n}>$ for all $i$, the apparent time of the ith clock is equal to the average of the time estimates computed at to plus the average of the time offsets measured to all other clocks. Finally, in a system with two clocks and $<$ EW sub i ( tau ) $)^{\sim} 1 / 2>$ for each, and if the estimated time at epoch $<$ \$Et sub $0^{\sim}+{ }^{\sim}$ tau> is fast by $1 \mathrm{~s}$ for one clock and slow by $1 \mathrm{~s}$ for the other, the apparent time for both clocks will coincide with the standard timescale.

In order to establish a basis for the next interval <\$Etau>, it is necessary to update the frequency estimate <\$ER hat sub i ( $t$ sub $0^{\sim}+{ }^{\sim}$ tau ) $>$ and weight factor <\$Ew sub i ( tau ) >. The average frequency assumed for the ith clock during the previous interval <\$Etau> is simply the difference between the times at the beginning and end of the interval divided by <\$Etau>. A good estimator for <\$ER sub i (t sub 0 + tau ) > has been found to be the exponential average of these differences, which is given by

$<$ \$ER hat sub $i\left(t \text { sub } 0^{\sim}+\sim \text { tau }\right)^{\sim}=\sim R$ hat sub $i$ (t sub 0 ) ${ }^{\sim}{ }^{\sim a l p h a}$ sub $i$ [ $R$ hat sub $i(t \text { sub } 0)^{\sim}-^{\sim}\left\{T \text { sub } i \text { ( } t \text { sub } 0^{\sim}+{ }^{\sim} \text { tau }\right)^{\sim}{ }^{\sim} T$ sub $i$ ( $t$ sub 0 ) $\}$ over tau ]>,

where <\$Ealpha sub i> is an experimentally determined weight factor which depends on the estimated frequency error of the ith clock. In order to calculate the weight factor <\$Ew sub i ( tau ) >, it is necessary to determine the expected error <\$Eepsilon sub i ( tau ) > for each clock. In the following, braces $\langle 169\rangle \mid\langle 170\rangle$ indicate absolute value and brackets $\langle 169\rangle\langle<>><170\rangle$ indicate the infinite time average. In practice, the infinite averages are computed as exponential time averages. An estimate of the magnitude of the unbiased error of the ith clock accumulated over the nominal interval <\$Etau> is

$<$ Eepsilon sub i ( tau ) ${ }^{\sim} \sim \mid T$ hat sub $i\left(t \text { sub } 0^{\sim}+{ }^{\sim} \text { tau }\right)^{\sim}-^{\sim} \mathrm{T}$ sub $i(t$ sub $0^{\sim}+$ tau ) $\mid \sim+\sim\left\{0.8^{\sim}<<\right.$ epsilon sub e $\sup 2$ ( tau ) $>>$ over sqrt \{ $<<^{\sim}$ epsilon sub $i \sup 2$ ( tau $\left.)^{\sim}>>\right\}>$,

where <\$Eepsilon sub i ( tau )> and <\$Eepsilon sub e ( tau ) > are the accumulated error of the ith clock and entire clock ensemble, respectively. The accumulated error of the entire ensemble is

$<\mathrm{E}<<\sim \operatorname{epsilon}$ sub e sup 2 ( tau $)^{\sim}>>^{\sim}=^{\sim}$ left $\left[\right.$ sum from $i=1$ to $\mathrm{n}^{\sim}$ over 
$<\sim$ epsilon sub i sup 2 ( tau $\left.)^{\sim}>>\right\}$ right ] $\sup \{\sim-1\}>$.

Finally, the weight factor for the ith clock is calculated as

$<$ \$EW sub i ( tau $)^{\sim}=^{\sim}\{<<\text { epsilon sub e sup } 2 \text { ( tau })^{\sim}>>$ over \{ $<<$ epsilon sub $i \sup 2$ ( tau $\left.)^{\sim}>>\right\}>$.

When all estimators and weight factors have been updated, the origin of the estimation interval is shifted and the new value of to becomes the old value of <\$Et sub $0^{\sim}+^{\sim}$ tau>.

While not entering into the above calculations, it is useful to estimate the frequency error, since the ensemble clocks can be located some distance from each other and become isolated for some time due to network failures. The frequency-offset error in $\mathrm{Ri}$ is equivalent to the fractional frequency yi,

$<$ Ey sub $i^{\sim}=^{\sim}\left\{\right.$ nu sub $i^{\sim}-^{\sim}$ nu sub $\left.I\right\}$ over nu sub I $>$

measured between the ith timescale and the standard timescale I. Temporarily dropping the subscript i for clarity, consider a sequence of $\mathrm{N}$ independent frequency-offset samples $\left\langle \$ \mathrm{Ey}(j) \sim\left(j^{\sim}={ }^{\sim} 1, \sim 2, \sim \ldots, \sim N\right)\right\rangle$ where the interval between samples is uniform and equal to $\mathrm{T}$. Let $<$ Stau> be the nominal interval over which these samples are averaged. The Allan variance <\$Esigma sub y sup $2(\mathrm{~N}, \sim \mathrm{T}, \sim$ tau $)>$ [ALL74a] is defined as $<$ E $<<$ sigma sub y sup $2(\mathrm{~N}, \sim \mathrm{T}, \sim \text { tau })^{\sim}>>^{\sim}=^{\sim}<<\sim 1$ over $\left\{\mathrm{N}^{\sim}-^{\sim} 1\right\}^{\sim}$ left [ sum from $j=1$ to $N^{\sim} y(j)$ sup $2^{\sim}-^{\sim} 1$ over $N^{\sim}$ left ( sum from $j=1$ to $N^{\sim} Y(j)$ right ) sup 2 right ] $>>>$,

A particularly useful formulation is $\left\langle \$ \mathrm{EN}^{\sim}=\sim 2>\right.$ and $\left\langle \$ \mathrm{ET}^{\sim} \mathbf{N}^{\sim}\right.$ tau $>$ :

$<\mathrm{E}<<$ sigma sub $\mathrm{y}$ sup $2\left(\mathrm{~N}^{\sim}=\sim 2, \sim \mathrm{T}^{\sim}=\sim\right.$ tau, $\sim$ tau $)>>^{\sim}==^{\sim}$ sigma sub y $\sup 2$ ( tau $) \sim{ }^{\sim}<<\left\{y\left(j \sim{ }^{\sim} 1\right) \sim \sim \mathcal{Y}(j)\right]$ sup 2$\}$ over $2>>>$,

so that

$<$ Esigma sub y sup 2 ( tau $)^{\sim}=\sim 1$ over $\left\{2\left(\mathrm{~N}^{\sim}-^{\sim} 1\right)\right\}$ sum from $\{j=1\}$ to $\{n-1\} \sim\left[y\left(j \sim{ }^{\sim} 1\right) \sim{ }^{\sim} y(j)\right]$ sup $2>$.

While the Allan variance has found application when estimating errors in ensembles of cesium clocks, its application to NTP is limited due to the computation and storage burden. As described in the next section, it is possible to estimate errors with some degree of confidence using normal byproducts of NTP processing algorithms.

Application to NTP

The NTP clock model is somewhat less complex than the general model described above. For instance, at the present level of development it is not necessary to separately estimate the time and frequency of all peer clocks, only the time and frequency of the local clock. If the timekeeping reference is the local clock itself, then the offsets available in the peer. offset peer variables can be used directly for the <\$ET sub ij> quantities above. In addition, the NTP local-clock model incorporates a type-II phase-locked loop, which itself reliably estimates frequency errors and corrects accordingly. Thus, the requirement for estimating frequency is entirely eliminated.

There remains the problem of how to determine a robust and easily computable error estimate <\$Eepsilon sub $i>$. The method described above, although analytically justified, is most difficult to implement. Happily, as a byproduct of the NTP clock-filter algorithm, a useful error estimate is available in the form of the dispersion. As described in the NTP specification, the dispersion includes the absolute value of the weighted average of the offsets between the chosen offset sample and the $<\$ E_{n} \sim \sim 1>$ other samples retained for selection. The effectiveness of 
this estimator was compared with the above estimator by simulation using observed timekeeping data and found to give quite acceptable results.

The NTP clock-combining algorithm can be implemented with only minor modifications to the algorithms as described in the NTP specification. Although elsewhere in the NTP specification the use of general-purpose multiply/divide routines has been successfully avoided, there seems to be no way to avoid them in the clock-combining algorithm. However, for best performance the local-clock algorithm described elsewhere in this document should be implemented as well, since the combining algorithms result in a modest increase in phase noise which the revised local-clock algorithm is designed to suppress.

Clock-Combining Procedure

The result of the NTP clock-selection procedure is a set of survivors (there must be at least one) that represent truechimers, or correct clocks. When clock combining is not implemented, one of these peers, chosen as the most likely candidate, becomes the synchronization source and its computed offset becomes the final clock correction.

Subsequently, the system variables are adjusted as described in the NTP clock-update procedure. When clock combining is implemented, these actions are unchanged, except that the final clock correction is computed by the clock-combining procedure.

The clock-combining procedure is called from the clock-select procedure. It constructs from the variables of all surviving peers the final clock correction <\$ETHETA>. The estimated error required by the algorithms previously described is based on the synchronization distance <\$ELAMBDA> computed by the distance procedure, as defined in the NTP specification. The reciprocal of <\$ELAMBDA> is the weight of each clock-offset contribution to the final clock correction. The following pseudo-code describes the procedure.

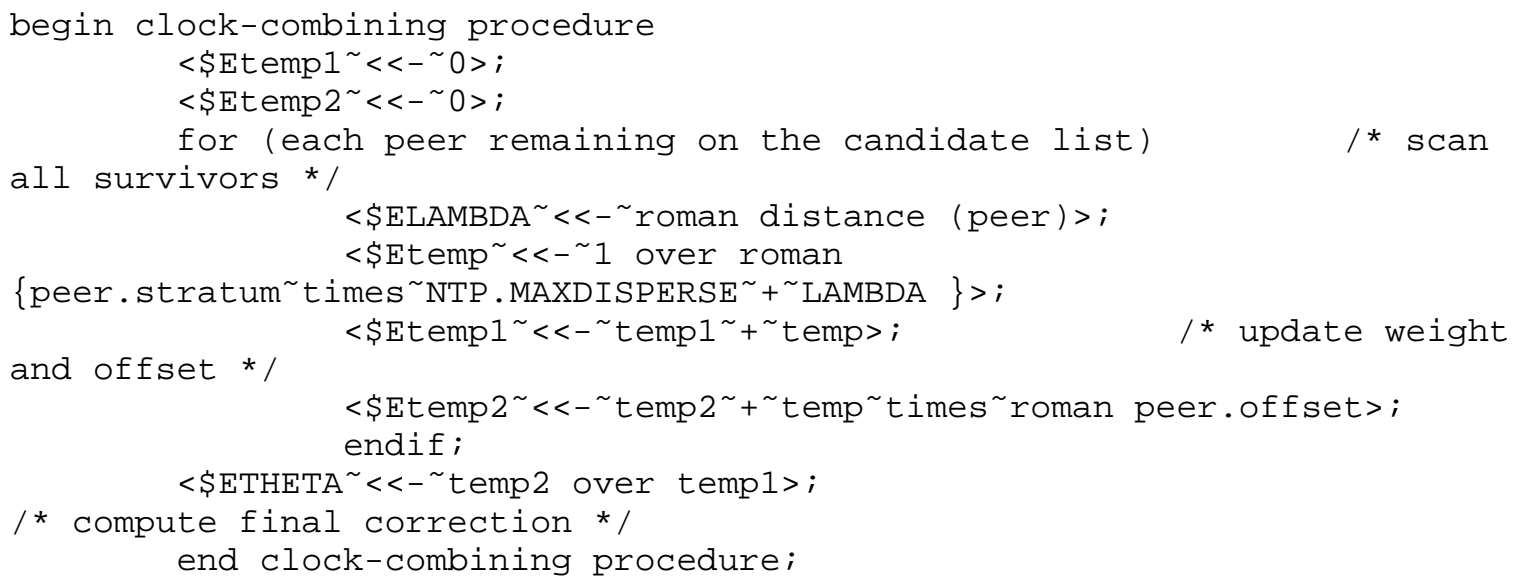

The value <\$ETHETA> is the final clock correction used by the localclock procedure to adjust the clock.

Appendix G. Computer Clock Modelling and Analysis

A computer clock includes some kind of reference oscillator, which is stabilized by a quartz crystal or some other means, such as the power grid. Usually, the clock includes a prescaler, which divides the oscillator frequency to a standard value, such as $1 \mathrm{MHz}$ or $100 \mathrm{~Hz}$, and a counter, implemented in hardware, software or some combination of the two, which can be read by the processor. For systems intended to be synchronized to an external source of standard time, there must be some means to correct the phase and frequency by occasional vernier adjustments produced by the timekeeping protocol. Special care is necessary in all timekeeping system designs to insure that the clock indications are always monotonically increasing; that is, system time never <169>runs backwards.<170> 
The simplest computer clock consists of a hardware latch which is set by overflow of a hardware counter or prescaler, and causes a processor interrupt or tick. The latch is reset when acknowledged by the processor, which then increments the value of a software clock counter. The phase of the clock is adjusted by adding periodic corrections to the counter as necessary. The frequency of the clock can be adjusted by changing the value of the increment itself, in order to make the clock run faster or slower. The precision of this simple clock model is limited to the tick interval, usually in the order of $10 \mathrm{~ms}$; although in some systems the tick interval can be changed using a kernel variable.

This software clock model requires a processor interrupt on every tick, which can cause significant overhead if the tick interval is small, say in the order less $1 \mathrm{~ms}$ with the newer RISC processors. Thus, in order to achieve timekeeping precisions less than $1 \mathrm{~ms}$, some kind of hardware assist is required. A straightforward design consists of a voltagecontrolled oscillator ( $\mathrm{VCO}$ ), in which the frequency is controlled by a buffered, digital/analog converter (DAC). Under the assumption that the VCO tolerance is 10-4 or 100 parts-per-million (ppm) (a reasonable value for inexpensive crystals) and the precision required is $100<$ Emu roman s> (a reasonable goal for a RISC processor), the DAC must include at least ten bits.

A design sketch of a computer clock constructed entirely of hardware logic components is shown in Figure $10 \mathrm{a}<\$ \& f i g 10>$. The clock is read by first pulsing the read signal, which latches the current value of the clock counter, then adding the contents of the clock-counter latch and a 64-bit clock-offset variable, which is maintained in processor memory. The clock phase is adjusted by adding a correction to the clock-offset variable, while the clock frequency is adjusted by loading a correction to the DAC latch. In principle, this clock model can be adapted to any precision by changing the number of bits of the prescaler or clock counter or changing the VCO frequency. However, it does not seem useful to reduce precision much below the minimum interrupt latency, which is in the low microseconds for a modern RISC processor.

If it is not possible to vary the oscillator frequency, which might be the case if the oscillator is an external frequency standard, a design such as shown in Figure $10 \mathrm{~b}$ may be used. It includes a fixed-frequency oscillator and prescaler which includes a dual-modulus swallow counter that can be operated in either divide-by-10 or divide-by-11 modes as controlled by a pulse produced by a programmable divider (PD). The PD is loaded with a value representing the frequency offset. Each time the divider overflows a pulse is produced which switches the swallow counter from the divide-by-10 mode to the divide-by-11 mode and then back again, which in effect $\langle 169>$ swallows $<170>$ or deletes a single pulse of the prescaler pulse train.

The pulse train produced by the prescaler is controlled precisely over a small range by the contents of the PD. If programmed to emit pulses at a low rate, relatively few pulses are swallowed per second and the frequency counted is near the upper limit of its range; while, if programmed to emit pulses at a high rate, relatively many pulses are swallowed and the frequency counted is near the lower limit. Assuming some degree of freedom in the choice of oscillator frequency and prescaler ratios, this design can compensate for a wide range of oscillator frequency tolerances.

In all of the above designs it is necessary to limit the amount of adjustment incorporated in any step to insure that the system clock indications are always monotonically increasing. With the software clock model this is assured as long as the increment is never negative. When the magnitude of a phase adjustment exceeds the tick interval (as corrected for the frequency adjustment), it is necessary to spread the 
adjustments over mulitple tick intervals. This strategy amounts to a deliberate frequency offset sustained for an interval equal to the total number of ticks required and, in fact, is a feature of the Unix clock model discussed below.

In the hardware clock models the same considerations apply; however, in these designs the tick interval amounts to a single pulse at the prescaler output, which may be in the order of $1 \mathrm{~ms}$. In order to avoid decreasing the indicated time when a negative phase correction occurs, it is necessary to avoid modifying the clock-offset variable in processor memory and to confine all adjustments to the VCO or prescaler. Thus, all phase adjustments must be performed by means of programmed frequency adjustments in much the same way as with the software clock model described previously.

It is interesting to conjecture on the design of a processor assist that could provide all of the above functions in a compact, general-purpose hardware interface. The interface might consist of a multifunction timer chip such as the AMD 9513A, which includes five 16-bit counters, each with programmable load and hold registers, plus an onboard crystal oscillator, prescaler and control circuitry. A 48-bit hardware clock counter would utilize three of the 16-bit counters, while the fourth would be used as the swallow counter and the fifth as the programmable divider. With the addition of a programmable-array logic device and architecture-specific host interface, this compact design could provide all the functions necessary for a comprehensive timekeeping system.

The Fuzzball Clock Model

The Fuzzball clock model uses a combination of hardware and software to provide precision timing with a minimum of software and processor overhead. The model includes an oscillator, prescaler and hardware counter; however, the oscillator frequency remains constant and the hardware counter produces only a fraction of the total number of bits required by the clock counter. A typical design uses a 64-bit software clock counter and a 16-bit hardware counter which counts the prescaler output. A hardware-counter overflow causes the processor to increment the software counter at the bit corresponding to the frequency <\$E2 sup $\mathrm{N}$ f sub $\mathrm{p}>$, where $\mathrm{N}$ is the number of bits of the hardware counter and $\mathrm{fp}$ is the counted frequency at the prescaler output. The processor reads the clock counter by first generating a read pulse, which latches the hardware counter, and then adding its contents, suitably aligned, to the software counter.

The Fuzzball clock can be corrected in phase by adding a (signed) adjustment to the software clock counter. In practice, this is done only when the local time is substantially different from the time indicated by the clock and may violate the monotonicity requirement. Vernier phase adjustments determined in normal system operation must be limited to no more than the period of the counted frequency, which is $1 \mathrm{kHz}$ for LSI-11 Fuzzballs. In the Fuzzball model these adjustments are performed at intervals of $4 \mathrm{~s}$, called the adjustment interval, which provides a maximum frequency adjustment range of $250 \mathrm{ppm}$. The adjustment opportunities are created using the interval-timer facility, which is a feature of most operating systems and independent of the time-of-day clock. However, if the counted frequency is increased from $1 \mathrm{kHz}$ to 1 $\mathrm{MHz}$ for enhanced precision, the adjustment frequency must be increased to $250 \mathrm{~Hz}$, which substantially increases processor overhead. A modified design suitable for high precision clocks is presented in the next section.

In some applications involving the Fuzzball model, an external pulseper-second (pps) signal is available from a reference source such as a cesium clock or GPS receiver. Such a signal generally provides much higher accuracy than the serial character string produced by a radio timecode receiver, typically in the low nanoseconds. In the Fuzzball model this signal is processed by an interface which produces a hardware 
interrupt coincident with the arrival of the pps pulse. The processor then reads the clock counter and computes the residual modulo $1 \mathrm{~s}$ of the clock counter. This represents the local-clock error relative to the pps signal.

Assuming the seconds numbering of the clock counter has been determined by a reliable source, such as a timecode receiver, the offset within the second is determined by the residual computed above. In the NTP localclock model the timecode receiver or NTP establishes the time to within $<$ F128M $>$ \& $<255 D>128 \mathrm{~ms}$, called the aperture, which guarantees the seconds numbering to within the second. Then, the pps residual can be used directly to correct the oscillator, since the offset must be less than the aperture for a correctly operating timecode receiver and pps signal.

The above technique has an inherent error equal to the latency of the interrupt system, which in modern RISC processors is in the low tens of microseconds. It is possible to improve accuracy by latching the hardware time-of-day counter directly by the pps pulse and then reading the counter in the same way as usual. This requires additional circuitry to prioritize the pps signal relative to the pulse generated by the program to latch the counter.

The Unix Clock Model

The Unix 4.3bsd clock model is based on two system calls, settimeofday and adjtime, together with two kernel variables tick and tickadj. The settimeofday call unceremoniously resets the kernel clock to the value given, while the adjtime call slews the kernel clock to a new value numerically equal to the sum of the present time of day and the (signed) argument given in the adjtime call. In order to understand the behavior of the Unix clock as controlled by the Fuzzball clock model described above, it is helpful to explore the operations of adjtime in more detail.

The Unix clock model assumes an interrupt produced by an onboard frequency source, such as the clock counter and prescaler described previously, to deliver a pulse train in the 100-Hz range. In priniciple, the power grid frequency can be used, although it is much less stable than a crystal oscillator. Each interrupt causes an increment called tick to be added to the clock counter. The value of the increment is chosen so that the clock counter, plus an initial offset established by the settimeofday call, is equal to the time of day in microseconds.

The Unix clock can actually run at three different rates, one corresponding to tick, which is related to the intrinsic frequency of the particular oscillator used as the clock source, one to <\$Etick ${ }^{\sim}{ }^{\sim}$ tickadj> and the third to <\$Etick ${ }^{\sim}{ }^{\text {tickadj }}$. Normally the rate corresponding to tick is used; but, if adjtime is called, the argument <\$Edelta> given is used to calculate an interval <\$EDELTA $t^{\sim}={ }^{\sim}$ delta ${ }^{\sim}$ tick over tickadj> during which one or the other of the two rates are used, depending on the sign of <\$Edelta>. The effect is to slew the clock to a new value at a small, constant rate, rather than incorporate the adjustment all at once, which could cause the clock to be set backward. With common values of $\left\langle \$ E_{t i c k}{ }^{\sim} \sim 10\right\rangle \mathrm{ms}$ and $<$ Etickadj $\sim$ 5 $^{\sim}$ mu roman $s>$, the maximum frequency adjustment range is $<$ E+- tickadj over $\mathrm{tick}^{\sim}=^{\sim}+-\left\{5^{\sim}\right.$ roman $\mathrm{x}^{\sim} 10$ sup -6$\}$ over $\{10$ sup -2$\}>$ or $<$ F128M $>æ<$ F255D>500 ppm. Even larger ranges may be required in the case of some workstations (e.g., SPARCstations) with extremely poor component tolerances.

When precisions not less than about $1 \mathrm{~ms}$ are required, the Fuzzball clock model can be adapted to the Unix model by software simulation, as described in Section 5 of the NTP specification, and calling adjtime at each adjustment interval. When precisions substantially better than this are required, the hardware microsecond clock provided in some workstations can be used together with certain refinements of the Fuzzball and Unix clock models. The particular design described below is appropriate for a maximum oscillator frequency tolerance of 100 ppm 
(.01\%), which can be obtained using a relatively inexpensive quartz crystal oscillator, but is readily scalable for other assumed tolerances.

The clock model requires the capability to slew the clock frequency over the range $\langle\mathrm{F} 128 \mathrm{M}>\Re<\mathrm{F} 255 \mathrm{D}>100 \mathrm{ppm}$ with an intrinsic oscillator frequency error as great as <F128M $>æ<F 255 D>100$ ppm. Figure $11<\$ \& f i g 11>$ shows the timing relationships at the extremes of the requirements envelope. Starting from an assumed offset of nominal zero and an assumed error of $+100 \mathrm{ppm}$ at time $0 \mathrm{~s}$, the line AC shows how the uncorrected offset grows with time. Let <\$Esigma> represent the adjustment interval and a the interval $A B$, in seconds, and let $r$ be the slew, or rate at which corrections are introduced, in ppm. For an accuracy specification of 100 $<$ Emu roman $\mathrm{s}>$, then

$<$ Esigma $<<=\sim\left\{100^{\sim m u}\right.$ roman $\left.\mathrm{s}\right\}$ over $\left\{100^{\sim} \text { roman ppm }\right\}^{\sim}+\sim 100^{\sim} \mathrm{mu}$ roman $\left.\mathrm{s}\right\}$

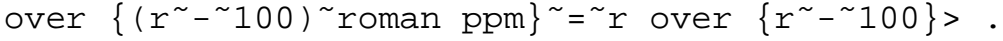

The line AE represents the extreme case where the clock is to be steered $<$ F128M $>-<$ F255D>100 ppm. Since the slew must be complete at the end of the adjustment interval,

$<$ Ea $\sim<=\sim\left\{\left(r^{\sim}-\sim 200\right) \sim\right.$ sigma $\}$ over $r>$.

These relationships are satisfied only if $\left\langle \$ E^{\sim}\right\rangle>^{\sim} 200^{\sim}$ roman ppm $\rangle$ and $<$ Esigma $<<2^{\sim}$ roman $\mathrm{s}>$. Using $<\$ \mathrm{Er}^{\sim}=\sim 300^{\sim}$ roman ppm $>$ for convenience, $<$ Esigma ${ }^{\sim} \sim 1.5^{\sim}$ roman $\mathrm{s}>$ and $<\$ \mathrm{Ea}^{\sim}<<=\sim 0.5^{\sim}$ roman $\mathrm{s}>$. For the Unix clock model with $\left\langle \$ E i \mathrm{ck}^{\sim}={ }^{\sim} 10^{\sim}\right.$ roman $\mathrm{ms}>$, this results in the value of $<$ Etickadj $\sim \sim 3^{\sim m u}$ roman $s>$.

One of the assumptions made in the Unix clock model is that the period of adjustment computed in the adjtime call must be completed before the next call is made. If not, this results in an error message to the system log. However, in order to correct for the intrinsic frequency offset of the clock oscillator, the NTP clock model requires adjtime to be called at regular adjustment intervals of <\$Esigma> $\mathrm{s}$. Using the algorithms described here and the architecture constants in the NTP specification, these adjustments will always complete.

Mathematical Model of the NTP Logical Clock

The NTP logical clock can be represented by the feedback-control model shown in Figure 12<\$\&fig12>. The model consists of an adaptiveparameter, phase-lock loop (PLL), which continuously adjusts the phase and frequency of an oscillator to compensate for its intrinsic jitter, wander and drift. A mathematical analysis of this model developed along the lines of [SMI86] is presented in following sections, along with a design example useful for implementation guidance in operating-systems environments such as Unix and Fuzzball. Table 9<\$\&tab9> summarizes the quantities ordinarily treated as variables in the model. By convention, $<\$ E V\rangle$ is used for internal loop variables, <\$Etheta> for phase, $<$ Eomega $>$ for frequency and <\$Etau> for time. Table 10<\$\&tab10> summarizes those quantities ordinarily fixed as constants in the model. Note that these are all expressed as a power of two in order to simplify the implementation.

In Figure 12 the variable <\$Etheta sub $r>$ represents the phase of the reference signal and <\$Etheta sub o> the phase of the voltage-controlled oscillator (VCO). The phase detector (PD) produces a voltage <\$Ev sub d> representing the phase difference <\$Etheta sub $r^{\sim}{ }^{\sim}$ theta sub o>. The clock filter functions as a tapped delay line, with the output <\$Ev sub s> taken at the tap selected by the clock-filter algorithm described in the NTP specification. The loop filter, represented by the equations given below, produces a VCO correction voltage $\langle \$ E v$ sub $c>$, which controls the oscillator frequency and thus the phase <\$Etheta sub o>.

The PLL behavior is completely determined by its open-loop, Laplace transfer function $<\$ E G(s)>$ in the $s$ domain. Since both frequency and 
phase corrections are required, an appropriate design consists of a type-II PLL, which is defined by the function

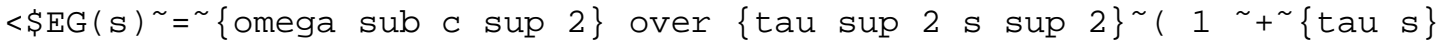
over omega sub z ) >,

where <\$Eomega sub $\mathrm{c}>$ is the crossover frequency (also called loop gain), <\$Eomega sub $z>$ is the corner frequency (required for loop stability) and <\$Etau> determines the PLL time constant and thus the bandwidth. While this is a first-order function and some improvement in phase noise might be gained from a higher-order function, in practice the improvement is lost due to the effects of the clock-filter delay, as described below.

The open-loop transfer function $<\$ E G(s)>$ is constructed by breaking the loop at point a on Figure 12 and computing the ratio of the output phase $<$ Etheta sub $\circ(\mathrm{s})>$ to the reference phase <\$Etheta sub $r(\mathrm{~s})>$. This function is the product of the individual transfer functions for the phase detector, clock filter, loop filter and VCO. The phase detector delivers a voltage $\left\langle \$ E V\right.$ sub $d(t)^{\sim}=^{\sim}$ theta sub $r(t)>$, so its transfer function is simply $<\$ E F$ sub $d(s)^{\sim}=\sim 1>$, expressed in $\mathrm{V} / \mathrm{rad}$. The VCO delivers a frequency change $<\$ E D E L T A$ omega $\sim \sim \sim$ roman $d^{\sim}$ theta sub $\left.o(t)\right\}$ over $\{\text { roman } d t\}^{\sim}=\sim$ alpha $\{\mathrm{V}$ sub $\mathrm{c}(t)\}>$, where $\langle \$ \mathrm{Ealph}$, is the VCO gain in rad/V-sec and <\$Etheta sub $O(t) \sim)^{\sim}$ alpha int $v$ sub $c(t) \sim d t>$. Its transfer function is the Laplace transform of the integral, <\$EF sub 0 $(\mathrm{s}) \sim=$ alpha over $\mathrm{s}>$, expressed in rad/V. The clock filter contributes a stochastic delay due to the clock-filter algorithm; but, for present purposes, this delay will be assumed a constant $T$, so its transfer function is the Laplace transform of the delay, <\$EF sub $s$ (s) $\sim \sim{ }^{\sim}$ sup $\{-\mathrm{TS}\}>$. Let $\langle \$ E F(S)\rangle$ be the transfer function of the loop filter, which has yet to be determined. The open-loop transfer function $\langle \$ E G(s)\rangle$ is the product of these four individual transfer functions:

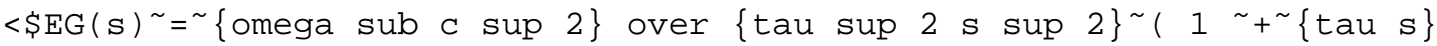
over omega sub $z$ ) ${ }^{\sim}=\sim$ F sub d (s) F sub $s(s) F(s)$ F sub o (s) $\sim \sim^{\sim} 1 e$ sup $\{-\mathrm{Ts}\} \sim \mathrm{F}(\mathrm{s}) \sim$ alpha over $\mathrm{s}>$.

For the moment, assume that the product <\$ETs> is small, so that $<\$ E e$ sup $\{-$ Ts $\}$ approx $\sim 1>$. Making the following substitutions,

$<$ Eomega sub $\mathrm{C}$ sup $2^{\sim}=\sim$ alpha over $\{\mathrm{K}$ sub $\mathrm{f}\} \sim \sim \sim \sim$ and $\left\langle \$ \mathrm{E}^{\sim \sim \sim \sim}\right.$ omega sub $\mathrm{z}^{\sim}=\sim \mathrm{K}$ sub $g$ over $\{\mathrm{K}$ sub $\mathrm{f}\}>$

and rearranging yields

$<\operatorname{SEF}(\mathrm{s})^{\sim}=^{\sim} 1$ over $\left\{\mathrm{K} \text { sub } \mathrm{g}^{\sim \text { tau }}\right\}^{\sim}+^{\sim} 1$ over $\left\{\mathrm{K}\right.$ sub $\mathrm{f}^{\sim \text { tau }}$ sup $\left.2 \mathrm{~s}\right\}>$,

which corresponds to a constant term plus an integrating term scaled by the PLL time constant <\$Etau>. This form is convenient for implementation as a sampled-data system, as described later.

With the parameter values given in Table 10, the Bode plot of the openloop transfer function $<\$ E G(s)>$ consists of a <196>12 dB/octave line which intersects the $0-d B$ baseline at $<\$$ Eomega sub $c^{\sim}=\sim 2$ sup $-12>\mathrm{rad} / \mathrm{s}$, together with a $+6 \mathrm{~dB} /$ octave line at the corner frequency <\$Eomega sub $\mathrm{z}^{\sim}=\sim 2$ sup $-14>\mathrm{rad} / \mathrm{s}$. The damping factor <\$Ezeta ${ }^{\sim}$ omega sub $\mathrm{c}$ over $\{2$ omega sub $z\}^{\sim}=\sim 2>$ suggests the PLL will be stable and have a large phase margin together with a low overshoot. However, if the clock-filter delay $T$ is not small compared to the loop delay, which is approximately equal to <\$E1 over omega sub $c>$, the above analysis becomes unreliable and the loop can become unstable. With the values determined as above, $T$ is ordinarily small enough to be neglected.

Assuming the output is taken at $\langle \$ E v$ sub $s>$, the closed-loop transfer function $\langle \$ E H(s)\rangle$ is

$<\$ E H(s)^{\sim}==^{\sim}\{\mathrm{v}$ sub $\mathrm{s}(\mathrm{s})\}$ over $\{\text { theta sub } r(\mathrm{~s})\}^{\sim}=^{\sim}\{\mathrm{F}$ sub $\mathrm{d}$ (s) e $\sup \{-$ 
Ts $\}$ over $\left\{1^{\sim}+\sim(S)\right\}>$.

If only the relative response is needed and the clock-filter delay can be neglected, $<\$ E H(s)>$ can be written

$<\$ E H(S)^{\sim}={ }^{\sim} 1$ over $\left\{1^{\sim}+{ }^{\sim} G(S)\right\}^{\sim}=\sim s$ sup 2 over $\left\{s\right.$ sup $2^{\sim}+^{\sim}$ omega sub $\mathrm{C}$ sup 2 over $\left\{\right.$ omega sub $\mathrm{z}^{\sim}$ tau\} $\mathrm{s}^{\sim}{ }^{\sim}$ omega sub $\mathrm{c}$ sup 2 over tau sup 2$\}>$.

For some input function $\langle$ SEI (s) $\rangle$ the output function $\langle \$ E I(\mathrm{~S}) \mathrm{H}(\mathrm{s})\rangle$ can be inverted to find the time response. Using a unit-step input $<\$ E I(S) \sim=^{\sim} 1$ over $s>$ and the values determined as above, This yields a PLL risetime of about 52 minutes, a maximum overshoot of about 4.8 percent in about 1.7 hours and a settling time to within one percent of the initial offset in about 8.7 hours.

Parameter Management

A very important feature of the NTP PLL design is the ability to adapt its behavior to match the prevailing stability of the local oscillator and transmission conditions in the network. This is done using the $<$ Ealpha> and <\$Etau> parameters shown in Table 10. Mechanisms for doing this are described in following sections.

Adjusting VCO Gain (<\$Ebold alpha>)

The <\$Ealpha> parameter is determined by the maximum frequency tolerance of the local oscillator and the maximum jitter requirements of the timekeeping system. This parameter is usually an architecture constant and fixed during system operation. In the implementation model described below, the reciprocal of <\$Ealpha>, called the adjustment interval <\$Esigma>, determines the time between corrections of the local clock, and thus the value of <\$Ealpha>. The value of <\$Esigma can be determined by the following procedure.

The maximum frequency tolerance for board-mounted, uncompensated quartzcrystal oscillators is probably in the range of 10-4 (100 ppm). Many if not most Internet timekeeping systems can tolerate jitter to at least the order of the intrinsic local-clock resolution, called precision in the NTP specification, which is commonly in the range from one to $20 \mathrm{~ms}$. Assuming 10-3 s peak-to-peak as the most demanding case, the interval between clock corrections must be no more than <\$Esigma ${ }^{\sim} \sim 10$ sup -3 over $\left\{2 \text { roman } \sim x^{\sim 10} \text { sup }-4\right\}^{\sim}=\sim 5>$ sec. For the NTP reference model $<$ Esigma $\sim \sim 4$ sec in order to allow for known features of the Unix operating-system kernel. However, in order to support future anticipated improvements in accuracy possible with faster workstations, it may be useful to decrease <\$Esigma> to as little as one-tenth the present value.

Note that if <\$Esigma> is changed, it is necessary to adjust the parameters <\$EK sub $\mathrm{f}>$ and $<\$ E K$ sub $g>$ in order to retain the same loop bandwidth; in particular, the same <\$Eomega sub $\mathrm{c}>$ and <\$Eomega sub $\mathrm{z}>$. Since <\$Ealpha> varies as the reciprocal of <\$Esigma>, if <\$Esigma> is changed to something other than 22, as in Table 10, it is necessary to divide both <\$EK sub $\mathrm{f}>$ and <\$EK sub $g>$ by <\$Esigma over $4>$ to obtain the new values.

Adjusting PLL Bandwidth (<\$Ebold tau $>$ )

A key feature of the type-II PLL design is its capability to compensate for the intrinsic frequency errors of the local oscillator. This requires a initial period of adaptation in order to refine the frequency estimate (see later sections of this appendix). The <\$Etau> parameter determines the PLL time constant and thus the loop bandwidth, which is approximately equal to <\$E\{omega sub c \} over tau>. When operated with a relatively large bandwidth (small <\$Etau>), as in the analysis above, the PLL adapts quickly to changes in the input reference signal, but has poor long term stability. Thus, it is possible to accumulate substantial errors if the system is deprived of the reference signal for an extended 
period. When operated with a relatively small bandwidth (large <\$Etau>), the PLL adapts slowly to changes in the input reference signal, and may even fail to lock onto it. Assuming the frequency estimate has stabilized, it is possible for the PLL to coast for an extended period without external corrections and without accumulating significant error.

In order to achieve the best performance without requiring individual tailoring of the loop bandwidth, it is necessary to compute each value of <\$Etau> based on the measured values of offset, delay and dispersion, as produced by the NTP protocol itself. The traditional way of doing this in precision timekeeping systems based on cesium clocks, is to relate <\$Etau> to the Allan variance, which is defined as the mean of the first-order differences of sequential samples measured during a specified interval <\$Etau>,

$<$ Esigma sub y sup 2 ( tau $)^{\sim}=\sim 1$ over $\left\{2\left(\mathrm{~N}^{\sim}-^{\sim} 1\right)\right\}$ sum from $\{i=1\}$ to $\{\mathrm{N}-1\} \sim\left[\mathrm{y}\left(\mathrm{i}^{\sim}+\sim 1\right) \sim{ }^{\sim} \mathrm{Y}(\mathrm{i})\right] \sup 2>$,

where $y$ is the fractional frequency measured with respect to the local timescale and $\mathrm{N}$ is the number of samples.

In the NTP local-clock model the Allan variance (called the compliance, $h$ in Table 11) is approximated on a continuous basis by exponentially averaging the first-order differences of the offset samples using an empirically determined averaging constant. Using somewhat ad-hoc mapping functions determined from simulation and experience, the compliance is manipulated to produce the loop time constant and update interval.

The NTP Clock Model

The PLL behavior can also be described by a set of recurrence equations, which depend upon several variables and constants. The variables and parameters used in these equations are shown in Tables 9, 10 and $11<\$ \& t a b 11>$. Note the use of powers of two, which facilitates implementation using arithmetic shifts and avoids the requirement for a multiply/divide capability.

A capsule overview of the design may be helpful in understanding how it operates. The logical clock is continuously adjusted in small increments at fixed intervals of <\$Esigma>. The increments are determined while updating the variables shown in Tables 9 and 11, which are computed from received NTP messages as described in the NTP specification. Updates computed from these messages occur at discrete times as each is received. The intervals <\$Emu> between updates are variable and can range up to about 17 minutes. As part of update processing the compliance $\mathrm{h}$ is computed and used to adjust the PLL time constant $<$ Etau>. Finally, the update interval <\$Erho> for transmitted NTP messages is determined as a fixed multiple of <\$Etau>.

Updates are numbered from zero, with those in the neighborhood of the ith update shown in Figure 13<\$\&fig13>. All variables are initialized at $<\$ E{ }^{\sim}=\sim 0>$ to zero, except the time constant <\$Etau $(0)^{\sim}=^{\sim}$ tau>, poll interval <\$Emu (0) $\sim{ }^{\sim}$ tau> (from Table 10) and compliance $<\$ E h \quad(0)^{\sim}=\sim \mathrm{K}$ sub $s\rangle$. After an interval <\$Emu $\left.\left.(i)\rangle\left(\left\langle \$ E i^{\sim}\right\rangle\right\rangle^{\sim} 0\right\rangle\right)$ from the previous update the ith update arrives at time <\$Et(i)> including the time offset $<\$ E V$ sub $S(i)>$. Then, after an interval <\$Emu $\left(i^{\sim}+^{\sim} 1\right)>$ the $<\$ E i+1$ roman $t h>$ update arrives at time $\left\langle \$ E t\left(i^{\sim}+{ }^{\sim} 1\right)>\right.$ including the time offset $\left\langle \$ E V\right.$ sub $\left.s\left(i^{\sim}+1\right)\right\rangle$. When the update $\langle \$ E V$ sub $s(i)\rangle$ is received, the frequency error $\left\langle \$ E f\left(i \sim{ }^{\sim} 1\right)\right\rangle$ and phase error $\left\langle \$ E g\left(i^{\sim}+^{\sim} 1\right)\right\rangle$ are computed:

$<$ Ef $(i \sim+\sim 1) \sim=\sim f(i) \sim+\sim$ mu (i) $v$ sub $s$ (i) $\}$ over $\{$ tau (i) sup 2$\}>$ ,$\left\langle \$ E^{\sim \sim \sim \sim} \sim(i \sim+\sim 1) \sim{ }^{\sim}\{\right.$ sub $\mathrm{s}$ (i) $\}$ over $\{$ tau (i) $\}>$.

Note that these computations depend on the value of the time constant $<$ Etau (i)> and poll interval <\$Emu (i)> previously computed from the $<\$ E i-1$ roman $t h>$ update. Then, the time constant for the next interval 
is computed from the current value of the compliance <\$Eh(i)>

$<$ SEtau $\left(i^{\sim}+\sim 1\right) \sim \sim \operatorname{roman} \max \left[K\right.$ sub $\left.\mathrm{s}^{\sim}{ }^{\sim} \sim \sim \sim \mathrm{h}(\mathrm{i}) \mid, \sim 1\right]>$.

Next, using the new value of <\$Etau>, called <\$Etau prime> to avoid confusion, the poll interval is computed

$<$ Erho $\left(i^{\sim}+\sim 1\right)^{\sim}=\sim K$ sub u tau prime $>$.

Finally, the compliance $\left\langle \$ E h\left(i^{\sim}+1\right)\right\rangle$ is recomputed for use in the $<\$ E i+1$ roman th> update:

$<$ Eh $\left(i^{\sim}+\sim 1\right)^{\sim}=\sim h(i)^{\sim}+\sim K$ sub $t^{\sim}$ tau prime $v$ sub $s$ (i) ${ }^{\sim}{ }^{\sim} h(i)$ \}over $K$ sub $h>$.

The factor <\$Etau prime> in the above has the effect of adjusting the bandwidth of the PLL as a function of compliance. When the compliance has been low over some relatively long period, <\$Etau prime> is increased and the bandwidth is decreased. In this mode small timing fluctuations due to jitter in the network are suppressed and the PLL attains the most accurate frequency estimate. On the other hand, if the compliance becomes high due to greatly increased jitter or a systematic frequency offset, <\$Etau prime> is decreased and the bandwidth is increased. In this mode the PLL is most adaptive to transients which can occur due to reboot of the system or a major timing error. In order to maintain optimum stability, the poll interval <\$Erho> is varied directly with $<$ Etau>

A model suitable for simulation and parameter refinement can be constructed from the above recurrence relations. It is convenient to set the temporary variable $\left\langle \$ \mathrm{Ea}^{\sim}{ }^{\sim} \mathrm{g}\left(i^{\sim}+\sim 1\right)\right\rangle$. At each adjustment interval <\$Esigma> the quantity <\$Ea over $K$ sub $g^{\sim}+^{\sim}\left\{f\left(i^{\sim}+{ }^{\sim} 1\right)\right\}$ over $K$ sub $f>$ is added to the local-clock phase and the quantity <\$Ea over $\mathrm{K}$ sub $\mathrm{g}>$ is subtracted from a. For convenience, let $\mathrm{n}$ be the greatest integer in $<\$ E\{m u$ (i) $\}$ over sigma>; that is, the number of adjustments that occur in the ith interval. Thus, at the end of the ith interval just before the <\$Ei+1 roman th> update, the VCO control voltage is:

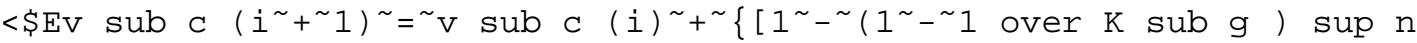
] $\left.\} \sim g\left(i^{\sim}+\sim 1\right)\right\} \sim+\sim \operatorname{over}\{\mathrm{K}$ sub $\mathrm{f}\} \sim\left\{f\left(i^{\sim}+\sim 1\right)\right\} \sim$.>

Detailed simulation of the NTP PLL with the values specified in Tables 9, 10 and 11 and the clock filter described in the NTP specification results in the following characteristics: For a 100-ms phase change the loop reaches zero error in 39 minutes, overshoots $7 \mathrm{~ms}$ at 54 minutes and settles to less than $1 \mathrm{~ms}$ in about six hours. For a 50-ppm frequency change the loop reaches 1 ppm in about 16 hours and 0.1 ppm in about 26 hours. When the magnitude of correction exceeds a few milliseconds or a few ppm for more than a few updates, the compliance begins to increase, which causes the loop time constant and update interval to decrease. When the magnitude of correction falls below about 0.1 ppm for a few hours, the compliance begins to decrease, which causes the loop time constant and update interval to increase. The effect is to provide a broad capture range exceeding $4 \mathrm{~s}$ per day, yet the capability to resolve oscillator skew well below $1 \mathrm{~ms}$ per day. These characteristics are appropriate for typical crystal-controlled oscillators with or without temperature compensation or oven control.

Appendix H. Analysis of Errors and Correctness Principles

Introduction

This appendix contains an analysis of errors arising in the generation and processing of NTP timestamps and the determination of delays and offsets. It establishes error bounds as a function of measured roundtrip delay and dispersion to the root (primary reference source) of the synchronization subnet. It also discusses correctness assertions about these error bounds and the time-transfer, filtering and selection 
algorithms used in NTP.

The notation $\left\langle \$ \mathrm{EW}^{\sim}{ }^{\sim}[\mathrm{u}, \sim \mathrm{v}]>\right.$ in the following describes the interval in which $u$ is the lower limit and $v$ the upper limit, inclusive. Thus, $<\$ \mathrm{Eu}^{\sim}=\sim \min (\mathrm{w}) \sim<\tilde{\sim}^{\sim} \mathrm{V}^{\sim}=\sim \max (\mathrm{w})>$, and for scalar $\mathrm{a}$, $<\$ E \mathrm{w}^{\sim}+\sim \mathrm{a}^{\sim}=\sim\left[\mathrm{u}^{\sim}+\sim \mathrm{a}, \mathrm{v}^{\sim}+\sim \mathrm{a}\right]>$. Table 12<\$\&tab12> shows a summary of other notation used in the analysis. The notation $\left\langle \$ E<<\sim^{\sim} x^{\sim}\right\rangle>>$ designates the (infinite) average of $x$, which is usually approximated by an exponential average, while the notation <\$Ex hat> designates an estimator for $x$. The lower-case Greek letters <\$Etheta>, <\$Edelta> and <\$Eepsilon> are used to designate measurement data for the local clock to a peer clock, while the upper-case Greek letters <\$ETHETA>, <\$EDELTA> and <\$EEPSILON> are used to designate measurement data for the local clock relative to the primary reference source at the root of the synchronization subnet. Exceptions will be noted as they arise.

\section{Timestamp Errors}

The standard second (1 s) is defined as <169>9,192,631,770 periods of the radiation corresponding to the transition between the two hyperfine levels of the ground state of the cesium-133 atom<170> [ALL74b], which implies a granularity of about $1.1 \times 10-10 \mathrm{~s}$. Other intervals can be determined as rational multiples of $1 \mathrm{~s}$. While NTP time has an inherent resolution of about $2.3 \times 10-10 \mathrm{~s}$, local clocks ordinarily have resolutions much worse than this, so the inherent error in resolving NTP time relative to the 1 s can be neglected.

In this analysis the local clock is represented by a counter/divider which increments at intervals of $s$ seconds and is driven by an oscillator which operates at frequency $\left\langle \$ E f\right.$ sub $c^{\sim}{ }^{\sim} n$ over s> for some integer $\mathrm{n}$. A timestamp $\langle \$ \mathrm{ET}(\mathrm{t})\rangle$ is determined by reading the clock at an arbitrary time $t$ (the argument $t$ will be usually omitted for conciseness). Strictly speaking, s is not known exactly, but can be assumed bounded from above by the maximum reading error <\$Erho>. The reading error itself is represented by the random variable $r$ bounded by

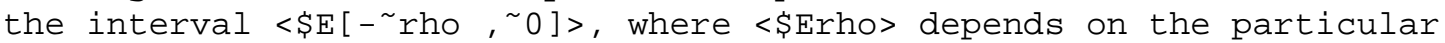
clock implementation. Since the intervals between reading the same clock are almost always independent of and much larger than $s$, successive readings can be considered independent and identically distributed. The frequency error of the clock oscillator is represented by the random variable $f$ bounded by the interval <\$E[- phi, $\sim$ phi ] , where <\$Ephi> represents the maximum frequency tolerance of the oscillator throughout its service life. While f for a particular clock is a random variable with respect to the population of all clocks, for any one clock it ordinarily changes only slowly with time and can usually be assumed a constant for that clock. Thus, an NTP timestamp can be represented by the random variable $\mathrm{T}$ :

$<\$ E T^{\sim}=\sim t^{\sim}+r^{\sim}+\sim$ tau $>$

where $t$ represents a clock reading, <\$Etau> represents the time interval since this reading and minor approximations inherent in the measurement of <\$Etau> are neglected.

In order to assess the nature and expected magnitude of timestamp errors and the calculations based on them, it is useful to examine the characteristics of the probability density functions (pdf) <\$Ep sub r $(x)>$ and $<\$ E p$ sub $f(x)>$ for $r$ and $f$ respectively. Assuming the clock reading and counting processes are independent, the pdf for $r$ is uniform over the interval <\$E[- rho, 0$]>$. With conventional manufacturing processes and temperature variations the pdf for $f$ can be approximated by a truncated, zero-mean Gaussian distribution with standard deviation $<$ \&sigma>. In conventional manufacturing processes <\$Esigma> is maneuvered so that the fraction of samples rejected outside the interval $<\$ E[-\sim p h i, \sim p h i]>$ is acceptable. The pdf for the total timestamp error $<$ Eepsilon $(x)>$ is thus the sum of the $r$ and $f$ contributions, computed as 
$<$ Eepsilon $(\mathrm{x})^{\sim} \mathbf{~}^{\sim}$ int ${ }^{\sim}$ from $\{-$ inf $\}$ to inf p sub $r$ (t) p sub $f\left(x^{\sim}-^{\sim} t\right)$

$d t>$,

which appears as a bell-shaped curve, symmetric about <\$E- rho over 2> and bounded by the interval

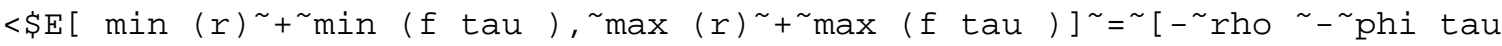
, phi tau ]>.

Since f changes only slowly over time for any single clock,

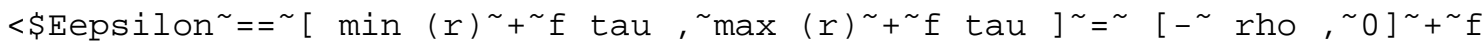
tau>,

where <\$Eepsilon> without argument designates the interval and $<$ Eepsilon $(\mathrm{x})>$ designates the pdf. In the following development subscripts will be used on various quantities to indicate to which entity or timestamp the quantity applies. Occasionally, <\$Eepsilon> will be used to designate an absolute maximum error, rather than the interval, but the distinction will be clear from context.

Measurement Errors

In NTP the roundtrip delay and clock offset between two peers A and B are determined by a procedure in which timestamps are exchanged via the network paths between them. The procedure involves the four most recent timestamps numbered as shown in Figure 14<\$\&fig14>, where the <\$Etheta sub 0> represents the true clock offset of peer $B$ relative to peer $A$. The <\$ET sub $1>$ and $<\$ E T$ sub $4>$ timestamps are determined relative to the A clock, while the <\$ET sub 2> and <\$ET sub 3> timestamps are determined relative to the B clock. The measured roundtrip delay $<$ SEdelta> and clock offset <\$Etheta> of B relative to A are given by

$<$ SEdelta ${ }^{\sim}\left(\mathrm{T}\right.$ sub 4 ${ }^{\sim} \sim \mathrm{T}$ sub 1$) \sim{ }^{\sim}\left(\mathrm{T}\right.$ sub 3 ${ }^{\sim}{ }^{\sim} \mathrm{T}$ sub 2 ) $\sim \sim$ and $\sim \sim \sim$ thet ${ }^{\sim}{ }^{\sim}\left\{\left(\mathrm{T}\right.\right.$ sub $2^{\sim}-^{\sim} \mathrm{T}$ sub 1$) \sim+^{\sim}\left(\mathrm{T}\right.$ sub $3^{\sim}-^{\sim} \mathrm{T}$ sub 4 ) $\}$ over $2>$.

The errors inherent in determining the timestamps $\mathrm{T} 1, \mathrm{~T} 2, \mathrm{~T} 3$ and $\mathrm{T} 4$ are, respectively,

<\$Eepsilon sub 1 ${ }^{\sim}{ }^{\sim}\left[-^{\sim}\right.$ rho sub $\left.\mathrm{A}, \sim 0\right]>, \quad\left\langle \$ E^{\sim}\right.$ epsilon sub $2^{\sim}=^{\sim}\left[-^{\sim}\right.$ rho sub B , 0]>, $<\$ E^{\sim}$ epsilon sub $3^{\sim}=\sim\left[-\sim\right.$ rho sub B, $\sim{ }^{\sim}{ }^{\sim}{ }^{\sim}$ f sub B $\left(T\right.$ sub $3 \sim-^{\sim T}$ sub 2 ) $>$, $<\$ E^{\sim}$ epsilon sub $4^{\sim}=^{\sim}\left[-^{\sim} \text { rho sub } A, \sim 0\right]^{\sim}+^{\sim}$ f sub A $\left(T\right.$ sub $4{ }^{\sim}{ }^{\sim}$ sub $1)>$.

For specific peers A and B, where <\$Ef sub A> and <\$Ef sub B> can be considered constants, the interval containing the maximum error inherent in determining <\$Edelta $>$ is given by

$<\$ E\left[\min (\text { epsilon sub } 4)^{\sim}{ }^{-}{ }^{\sim} \max (\operatorname{epsilon} \text { sub } 1)^{\sim}{ }^{-}{ }^{\sim m a x}(\operatorname{epsilon}\right.$ sub 3 )$^{\sim}+\sim \min \left(\right.$ epsilon sub 2 ), $\sim \max \left(\right.$ epsilon sub 4) ${ }^{\sim}{ }^{\sim}{ }^{\sim} \min (\operatorname{epsilon}$ sub 1 ) - $\min \left(\right.$ epsilon sub 3 ) ${ }^{+} \sim \max$ (epsilon sub 2 )] >

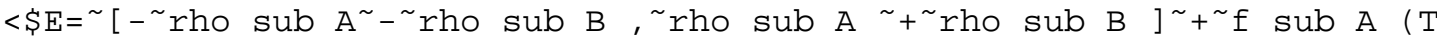
sub $4^{\sim}{ }^{\sim} \mathrm{T}$ sub 1$)^{\sim}{ }^{\sim}$ f sub $\mathrm{B}\left(\mathrm{T}\right.$ sub $3^{\sim}-^{\sim} \mathrm{T}$ sub 2$)>$.

In the NTP local clock model the residual frequency errors <\$Ef sub $A>$ and <\$Ef sub B> are minimized through the use of a type-II phase-lock loop (PLL). Under most conditions these errors will be small and can be ignored. The pdf for the remaining errors is symmetric, so that <\$Edelta hat $^{\sim}{ }^{\sim}<<$ delta $>>$ is an unbiased maximum-likelihood estimator for the true roundtrip delay, independent of the particular values of <\$Erho sub A $>$ and <\$Erho sub $B>$.

However, in order to reliably bound the errors under all conditions of component variation and operational regimes, the design of the PLL and the tolerance of its intrinsic oscillator must be controlled so that it is not possible under any circumstances for <\$Ef sub $A>$ or <\$Ef sub B> 
to exceed the bounds <\$E[- ${ }^{\sim}$ phi sub A, phi sub A ] $>$ or $<\$ E[-\sim$ phi sub B , phi sub B ]>, respectively. Setting <\$Erho ${ }^{\sim}$ max ( rho sub A, ${ }^{2}$ rho sub B )> for convenience, the absolute maximum error <\$Eepsilon sub delta> inherent in determining roundtrip delay <\$Edelta> is given by

<\$Eepsilon sub delta ${ }^{\sim}=\sim{ }^{\sim}$ rho ${ }^{\sim} \sim$ phi sub A ( $T$ sub $4{ }^{\sim}{ }^{\sim} T$ sub 1 ) ${ }^{\sim} \sim$ phi sub B ( $\mathrm{T}$ sub $3^{\sim}-^{\sim} \mathrm{T}$ sub 2 ) $>$,

neglecting residuals.

As in the case for <\$Edelta>, where <\$Ef sub $A>$ and <\$Ef sub B> can be considered constants, the interval containing the maximum error inherent in determining <\$Etheta> is given by

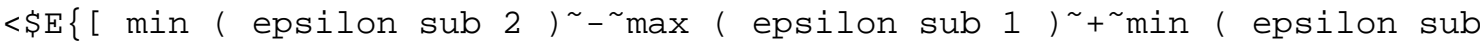
$3)^{\sim}{ }^{\sim} \max \left(\right.$ epsilon sub 4), $\sim \max \left(\right.$ epsilon sub 2 ) ${ }^{\sim}{ }^{\sim} \min$ ( epsilon sub $1)^{\sim}+\sim \max \left(\operatorname{epsilon}\right.$ sub 3 ) ${ }^{\sim} \sim \min$ (epsilon sub 4)]\} over 2>

$<\$ E=\sim[-\sim \text { rho sub B, rho sub } A]^{\sim}+^{\sim}\left\{f\right.$ sub B (T sub $3^{\sim}-^{\sim} T$ sub 2 ) $-^{\sim}$ f sub A ( T sub $4 \sim{ }^{\sim} \mathrm{T}$ sub 1 ) \} over $2>$.

Under most conditions the errors due to <\$Ef sub $A>$ and $<\$ E f$ sub $B>$ will be small and can be ignored. If <\$Erho sub $A^{\sim}={ }^{\sim}$ rho sub $B^{\sim}=^{\sim}$ rho>; that is, if both the $A$ and $B$ clocks have the same resolution, the pdf for the remaining errors is symmetric, so that $<$ Etheta hat ${ }^{\sim}{ }^{\sim}<<$ theta $>>>$ is an unbiased maximum-likelihood estimator for the true clock offset <\$Etheta sub 0>, independent of the particular value of <\$Erho>. If <\$Erho sub $\mathrm{A}^{\sim}$ ! = rho sub $\mathrm{B}>, \quad\langle\mathrm{SE}<<$ theta $>>>$ is not an unbiased estimator; however, the bias error is in the order of

$<\$ E\left\{\right.$ rho sub $A^{\sim}{ }^{\sim}$ rho sub $\left.B\right\}$ over $2>$.

and can usually be neglected.

Again setting <\$Erho ${ }^{\sim}$ max ( rho sub A, rho sub B ) > for convenience, the absolute maximum error <\$Eepsilon sub theta> inherent in determining clock offset <\$Etheta> is given by

$<$ Eepsilon sub theta ${ }^{\sim}==^{\sim}\left\{r h 0^{\sim}+\sim\right.$ phi sub A (T sub $4^{\sim}{ }^{\sim}{ }^{\sim}$ sub 1 ) $+^{\sim}$ phi sub B ( $\mathrm{T}$ sub $3^{\sim}-^{\sim} \mathrm{T}$ sub 2 ) \} over $2>$.

Network Errors

In practice, errors due to stochastic network delays usually dominate. In general, it is not possible to characterize network delays as a stationary random process, since network queues can grow and shrink in chaotic fashion and arriving customer traffic is frequently bursty. However, it is a simple exercise to calculate bounds on clock offset errors as a function of measured delay. Let $\left\langle \$ E T\right.$ sub $2^{\sim}-^{\sim} \mathrm{T}$ sub $\left.1^{\sim}=^{\sim} \mathrm{a}\right\rangle$ and $\left\langle\right.$ \$ET sub $3^{\sim}-^{\sim} \mathrm{T}$ sub $4^{\sim}=\sim \mathrm{b}>$. Then,

$<$ SEdelta $\sim \mathrm{a}^{\sim}-\sim \mathrm{b}^{\sim \sim \sim \sim}$ and $\sim \sim \sim$ thet $\mathrm{a}^{\sim}=\sim\left\{\mathrm{a}^{\sim}+\sim \mathrm{b}\right\}$ over $2>$.

The true offset of $B$ relative to $A$ is called <\$Etheta sub $0>$ in Figure 14. Let $x$ denote the actual delay between the departure of a message from $A$ and its arrival at $B$. Therefore, $<\$ E x^{\sim}+{ }^{\sim}$ theta sub $0^{\sim}=\sim^{\sim} \mathrm{T}$ sub $2^{\sim}-^{\sim} \mathrm{T}$ sub $1^{\sim}==\sim a>$. Since $x$ must be positive in our universe, $\left\langle \$ E x^{\sim}=\sim a^{\sim}-^{\sim}\right.$ theta sub $0^{\sim}>>=\sim 0>$, which requires $<$ Etheta sub $0^{\sim}<<=\sim a>$. A similar argument requires that $\left\langle \$ \mathrm{~Eb}^{\sim}<<=\sim\right.$ theta sub $0>$, so surely $<\$ E \mathrm{~b}^{\sim}<<=\sim$ theta sub $0 \sim<=\sim a>$. This inequality can also be expressed

$<\$ E \mathrm{~b}^{\sim}=^{\sim}\left\{\mathrm{a}^{\sim}+\sim \mathrm{b}\right\}$ over $2^{\sim}-^{\sim}\left\{\mathrm{a}^{\sim}-^{\sim} \mathrm{b}\right\}$ over $2^{\sim}<<=$ theta sub $\left.0^{\sim}<<=\sim \mathrm{a}^{\sim}+{ }^{\sim} \mathrm{b}\right\}$ over $\left.2^{\sim}+\sim a \sim-\sim b\right\}$ over $2^{\sim}=\sim a>$,

which is equivalent to

$<$ Stheta ${ }^{\sim}$ delta over $2^{\sim}<<=$ theta sub $0^{\sim}<<=$ theta ${ }^{\sim}$ delta over $2>$.

In the previous section bounds on delay and offset errors were 
determined. Thus, the inequality can be written

$<$ \$Etheta ${ }^{\sim}$ epsilon sub theta ${ }^{\sim}$ \{delta ${ }^{\sim}$ epsilon sub delta\} over

$2^{\sim}<<=\sim$ theta sub $0^{\sim}<<=\sim$ theta ${ }^{\sim}{ }^{\sim}$ epsilon sub theta ${ }^{\sim} \sim$ \{delta ${ }^{\sim}{ }^{\sim}$ epsilon sub

delta \} over $2>$,

where <\$Eepsilon sub theta> is the maximum offset error and <\$Eepsilon sub delta> is the maximum delay error derived previously. The quantity

$<$ Eepsilon ${ }^{\sim}$ epsilon sub theta ${ }^{+}$epsilon sub delta over $2^{\sim}{ }^{\sim}{ }^{\sim}$ rho ${ }^{\sim} \sim$ phi sub A ( $\mathrm{T}$ sub $4^{\sim}-^{\sim} \mathrm{T}$ sub 1$)^{\sim}{ }^{\sim}$ phi sub $B\left(T\right.$ sub $3^{\sim}-^{\sim} T$ sub 2$)>$,

called the peer dispersion, defines the maximum error in the inequality. Thus, the correctness interval I can be defined as the interval

$<\$ E I^{\sim}=\sim\left[\right.$ theta ${ }^{\sim} \sim \operatorname{delta}$ over $2^{\sim}-^{\sim}$ epsilon, theta $^{\sim}+$ delta over $2^{\sim}+{ }^{\sim}$ epsilon ]$>$,

in which the clock offset $\left\langle \$ E^{\sim} C^{\sim}\right.$ theta $>$ is the midpoint. By

construction, the true offset <\$Etheta sub $0>$ must lie somewhere in this interval.

Inherited Errors

As described in the NTP specification, the NTP time server maintains the local clock <\$ETHETA>, together with the root roundtrip delay <\$EDELTA> and root dispersion <\$EEPSILON> relative to the primary reference source at the root of the synchronization subnet. The values of these variables are either included in each update message or can be derived as described in the NTP specification. In addition, the protocol exchange and clock-filter algorithm provide the clock offset <\$Etheta> and roundtrip delay <\$Edelta> of the local clock relative to the peer clock, as well as various error accumulations as described below. The following discussion establishes how errors inherent in the time-transfer process accumulate within the subnet and contribute to the overall error budget at each server.

An NTP measurement update includes three parts: clock offset <\$Etheta>, roundtrip delay <\$Edelta> and maximum error or dispersion <\$Eepsilon> of the local clock relative to a peer clock. In case of a primary clock update, these values are usually all zero, although <\$Eepsilon> can be tailored to reflect the specified maximum error of the primary reference source itself. In other cases <\$Etheta> and <\$Edelta> are calculated directly from the four most recent timestamps, as described in the NTP specification. The dispersion <\$Eepsilon> includes the following contributions:

1.

Each time the local clock is read a reading error is incurred due to the finite granularity or precision of the implementation. This is called the measurement dispersion <\$Erho>.

2 .

Once an offset is determined, an error due to frequency offset or skew accumulates with time. This is called the skew dispersion <\$Ephi tau>, where <\$Ephi> represents the skew-rate constant (<\$Eroman NTP.MAXSKEW over NTP.MAXAGE> in the NTP specification) and <\$Etau> is the interval since the dispersion was last updated.

3

When a series of offsets are determined at regular intervals and accumulated in a window of samples, as in the NTP clock-filter algorithm, the (estimated) additional error due to offset sample variance is called the filter dispersion <\$Eepsilon sub sigma>. 
When a number of peers are considered for synchronization and two or more are determined to be correctly synchronized to a primary reference source, as in the NTP clock-selection algorithm, the (estimated) additional error due to offset sample variance is called the selection dispersion <\$Eepsilon sub xi>.

Figure 15<\$\&fig15> shows how these errors accumulate in the ordinary course of NTP processing. Received messages from a single peer are represented by the packet variables. From the four most recent timestamps T1, T2, T3 and T4 the clock offset and roundtrip delay sample for the local clock relative to the peer clock are calculated directly. Included in the message are the root roundtrip delay <\$EDELTA prime> and root dispersion <\$EEPSILON prime> of the peer itself; however, before sending, the peer adds the measurement dispersion <\$Erho> and skew dispersion <\$Ephi tau>, where these quantities are determined by the peer and <\$Etau> is the interval according to the peer clock since its clock was last updated.

The NTP clock-filter procedure saves the most recent samples <\$Etheta sub $i>$ and <\$Edelta sub $i>$ in the clock filter as described in the NTP specification. The quantities <\$Erho> and <\$Ephi> characterize the local clock maximum reading error and frequency error, respectively. Each sample includes the dispersion <\$Eepsilon sub $i^{\sim}={ }^{\sim}$ rho ${ }^{\sim}{ }^{\sim}$ phi $\left(\mathrm{T}\right.$ sub $4^{\sim}-^{\sim} \mathrm{T}$ sub 1 )>, which is set upon arrival. Each time a new sample arrives all samples in the filter are updated with the skew dispersion <\$Ephi tau sub $i>$, where <\$Etau sub $i>$ is the interval since the last sample arrived, as recorded in the variable peer.update. The clock-filter algorithm determines the selected clock offset <\$Etheta> (peer.offset), together with the associated roundtrip delay <\$Edelta> (peer.delay) and filter dispersion <\$Eepsilon sub sigma>, which is added to the associated sample dispersion <\$Eepsilon sub i> to form the peer dispersion <\$Eepsilon> (peer.dispersion).

The NTP clock-selection procedure selects a single peer to become the synchronization source as described in the NTP specification. The operation of the algorithm determines the final clock offset <\$ETHETA> (local clock), roundtrip delay <\$EDELTA> (sys.rootdelay) and dispersion $<$ EEPSILON> (sys.rootdispersion) relative to the root of the synchronization subnet, as shown in Figure 15. Note the inclusion of the selected peer dispersion and skew accumulation since the dispersion was last updated, as well as the select dispersion <\$Eepsilon sub $x i>$ computed by the clock-select algorithm itself. Also, note that, in order to preserve overall synchronization subnet stability, the final clock offset <\$ETHETA> is in fact determined from the offset of the local clock relative to the peer clock, rather than the root of the subnet. Finally, note that the packet variables <\$EDELTA prime> and <\$EEPSILON prime> are in fact determined from the latest message received, not at the precise time the offset selected by the clock-filter algorithm was determined. Minor errors arising due to these simplifications will be ignored. Thus, the total dispersion accumulation relative to the root of the synchronization subnet is

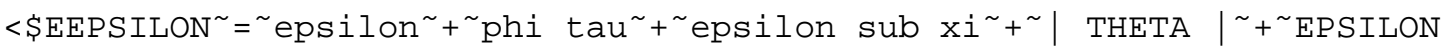
prime $>$,

where <\$Etau> is the time since the peer variables were last updated and $<\$ E \mid$ THETA $\mid>$ is the initial absolute error in setting the local clock.

The three values of clock offset, roundtrip delay and dispersion are all additive; that is, if <\$ETHETA sub i>, <\$EDELTA sub i> and <\$EEPSILON sub i> represent the values at peer i relative to the root of the synchronization subnet, the values

$<$ \$ETHETA sub $j(t) \sim={ }^{\sim}$ THETA sub $i^{\sim}+{ }^{\sim}$ theta sub $j(t)>, \quad<$ EDELTA sub $j$ $(t) \sim=\sim$ DELTA sub $i^{\sim}+\sim \operatorname{delta}$ sub $j>, \quad\left\langle \$ E E P S I L O N\right.$ sub $j(t) \sim={ }^{\sim}$ EPSILON 
sub i ${ }^{+}$epsilon sub $i^{\sim}{ }^{\sim}$ epsilon sub j $(t)>$,

represent the clock offset, roundtrip delay and dispersion of peer $j$ at time $t$. The time dependence of <\$Etheta sub $j(t)>$ and <\$Eepsilon sub $j$ $(t)>$ represents the local-clock correction and dispersion accumulated since the last update was received from peer $i$, while the term <\$Eepsilon sub i> represents the dispersion accumulated by peer i from the time its clock was last set until the latest update was sent to peer $j$. Note that, while the offset of the local clock relative to the peer clock can be determined directly, the offset relative to the root of the synchronization subnet is not directly determinable, except on a probabilistic basis and within the bounds established in this and the previous section.

The NTP synchronization subnet topology is that of a tree rooted at the primary server(s). Thus, there is an unbroken path from every time server to the primary reference source. Accuracy and stability are proportional to synchronization distance <\$ELAMBDA>, defined as

$<\$ E L A M B D A \sim=\sim$ EPSILON $^{\sim}+\sim$ DELTA over $2>$.

The selection algorithm favors the minimum-distance paths and thus maximizes accuracy and stability. Since <\$ETHETA sub 0>, <\$EDELTA sub 0> and <\$EEPSILON sub 0> are all zero, the sum of the clock offsets, roundtrip delays and dispersions of each server along the minimumdistance path from the root of the synchronization subnet to a given server $i$ are the clock offset <\$ETHETA sub i>, roundtrip delay <\$EDELTA sub i> and dispersion <\$EEPSILON sub i> inherited by and characteristic of that server.

Correctness Principles

In order to minimize the occurrence of errors due to incorrect clocks and maximize the reliability of the service, NTP relies on multiple peers and disjoint peer paths whenever possible. In the previous development it was shown that, if the primary reference source at the root of the synchronization subnet is in fact a correct clock, then the true offset <\$Etheta sub $0>$ relative to that clock must be contained in the interval

$<$ \$E [ THETA $\sim \sim$ LAMBDA, $\sim$ THETA $\sim{ }^{\sim}$ LAMBDA ] $==\sim[$ THETA $\sim-\sim$ EPSILON $\sim \sim$ DELTA over 2 , THETA $^{\sim}+\sim$ EPSILON ${ }^{\sim} \sim$ DELTA over 2 ] $>$.

When a number of clocks are involved, it is not clear beforehand which are correct and which are not; however, as cited previously, there are a number of techniques based on clustering and filtering principles which yield a high probability of detecting and discarding incorrect clocks. Marzullo and Owicki [MAR85] devised an algorithm designed to find an appropriate interval containing the correct time given the confidence intervals of $\mathrm{m}$ clocks, of which no more than $f$ are considered incorrect. The algorithm finds the smallest single intersection containing all points in at least $\left\langle \$ E^{\sim}{ }^{\sim}\right.$ f $>$ of the given confidence intervals.

Figure 16<\$\&fig16> illustrates the operation of this algorithm with a scenario involving four clocks A, B, C and D, with the calculated time (shown by the $\langle$ F128>i<F255> symbol) and confidence interval shown for each. These intervals are computed as described in previous sections of this appendix. For instance, any point in the A interval may possibly represent the actual time associated with that clock. If all clocks are correct, there must exist a nonempty intersection including all four intervals; but, clearly this is not the case in this scenario. However, if it is assumed that one of the clocks is incorrect (e.g., D), it might be possible to find a nonempty intersection including all but one of the intervals. If not, it might be possible to find a nonempty intersection including all but two of the intervals and so on.

The algorithm proposed by DEC for use in the Digital Time Service [DEC89] is based on these principles. For the scenario illustrated in 
Figure 16, it computes the interval for $<\$ \mathrm{Em}^{\sim}={ }^{\sim} 4>$ clocks, three of which turn out to be correct and one not. The low endpoint of the intersection is found as follows. A variable $f$ is initialized with the number of presumed incorrect clocks, in this case zero, and a counter i is initialized at zero. Starting from the lowest endpoint, the algorithm increments $i$ at each low endpoint, decrements $i$ at each high endpoint, and stops when $\left.\left.\left\langle \$ E E^{\sim}\right\rangle\right\rangle=\sim \mathrm{m}^{\sim}-\tilde{f}\right\rangle$. The counter records the number of intersections and thus the number of presumed correct clocks. In the example the counter never reaches four, so $f$ is increased by one and the procedure is repeated. This time the counter reaches three and stops at the low endpoint of the intersection marked DTS. The upper endpoint of this intersection is found using a similar procedure.

This algorithm will always find the smallest single intersection containing points in at least one of the original <\$Em ${ }^{\sim}{ }^{\sim}$ f $>$ confidence intervals as long as the number of incorrect clocks is less than half the total $<\$ E f^{\sim}<<^{\sim}$ over $2>$. However, some points in the intersection

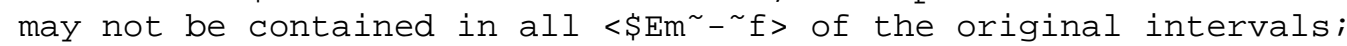
moreover, some or all of the calculated times (such as for C in Figure 16) may lie outside the intersection. In the NTP clock-selection procedure the above algorithm is modified so as to include at least $<\$ E m^{\sim} \sim^{\sim}$ > of the calculated times. In the modified algorithm a counter $C$ is initialized at zero. When starting from either endpoint, $c$ is incremented at each calculated time; however, neither $\mathrm{f}$ nor c are reset between finding the low and high endpoints of the intersection. If after both endpoints have been found $\left.\left.\left\langle \$ E^{\sim}{ }^{\sim}\right\rangle\right\rangle^{\sim} f\right\rangle$, $f$ is increased by one and the entire procedure is repeated. The revised algorithm finds the smallest intersection of $\left\langle \$ \mathrm{Em}^{\sim}-^{\sim} \mathrm{f}>\right.$ intervals containing at least $\left.<\$ \mathrm{Em}^{\sim}-^{\sim} \mathrm{f}\right\rangle$ calculated times. As shown in Figure 16, the modified algorithm produces the intersection marked NTP and including the calculated time for C.

In the NTP clock-selection procedure the peers represented by the clocks in the final intersection, called the survivors, are placed on a candidate list. In the remaining steps of the procedure one or more survivors may be discarded from the list as outlyers. Finally, the clock-combining algorithm described in Appendix F provides a weighted average of the remaining survivors based on synchronization distance. The resulting estimates represent a synthetic peer with offset between the maximum and minimum offsets of the remaining survivors. This defines the clock offset <\$ETHETA>, total roundtrip total delay <\$EDELTA> and total dispersion <\$EEPSILON> which the local clock inherits. In principle, these values could be included in the time interface provided by the operating system to the user, so that the user could evaluate the quality of indications directly.

Appendix I. Selected C-Language Program Listings

Following are $\mathrm{C}$-language program listings of selected algorithms described in the NTP specification. While these have been tested as part of a software simulator using data collected in regular operation, they do not necessarily represent a standard implementation, since many other implementations could in principle conform to the NTP specification.

Common Definitions and Variables

The following definitions are common to all procedures and peers.

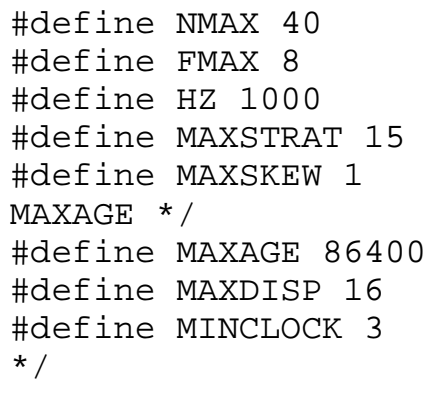

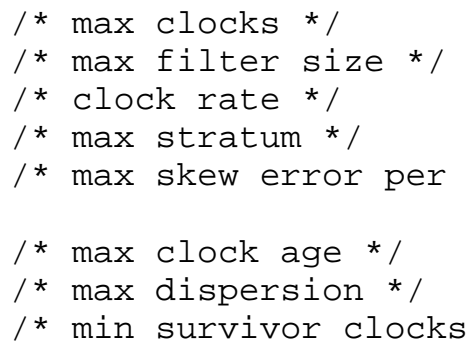




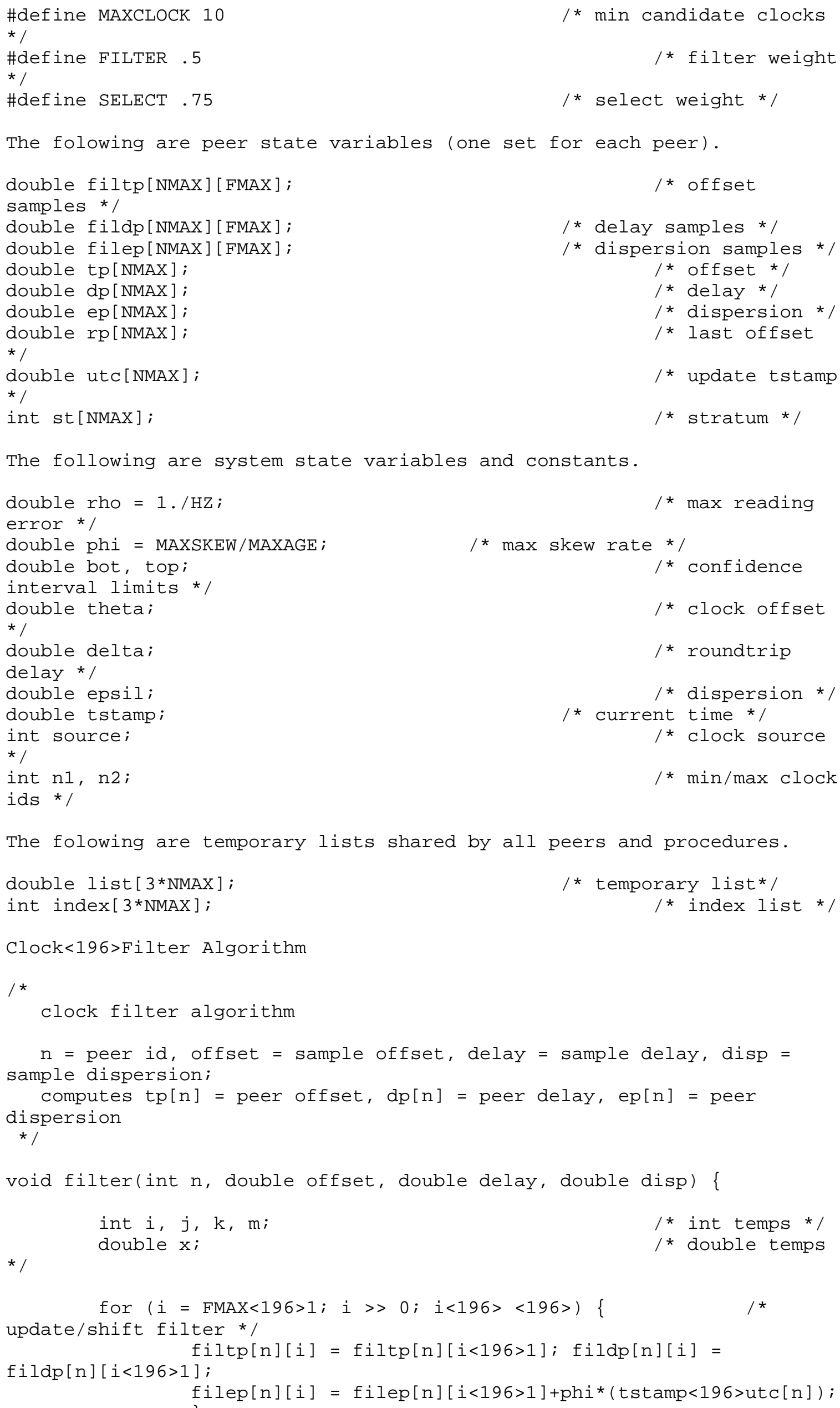




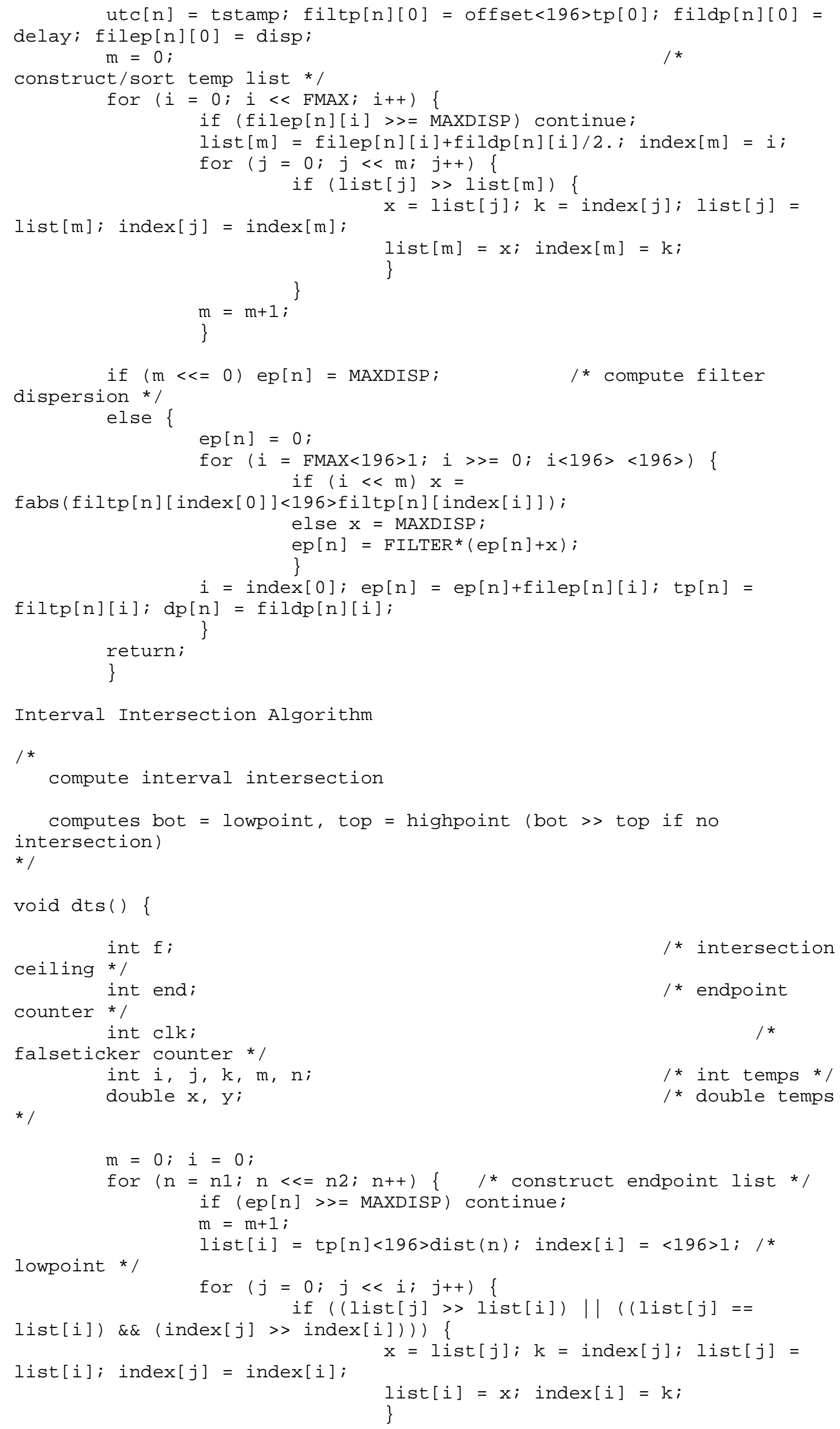




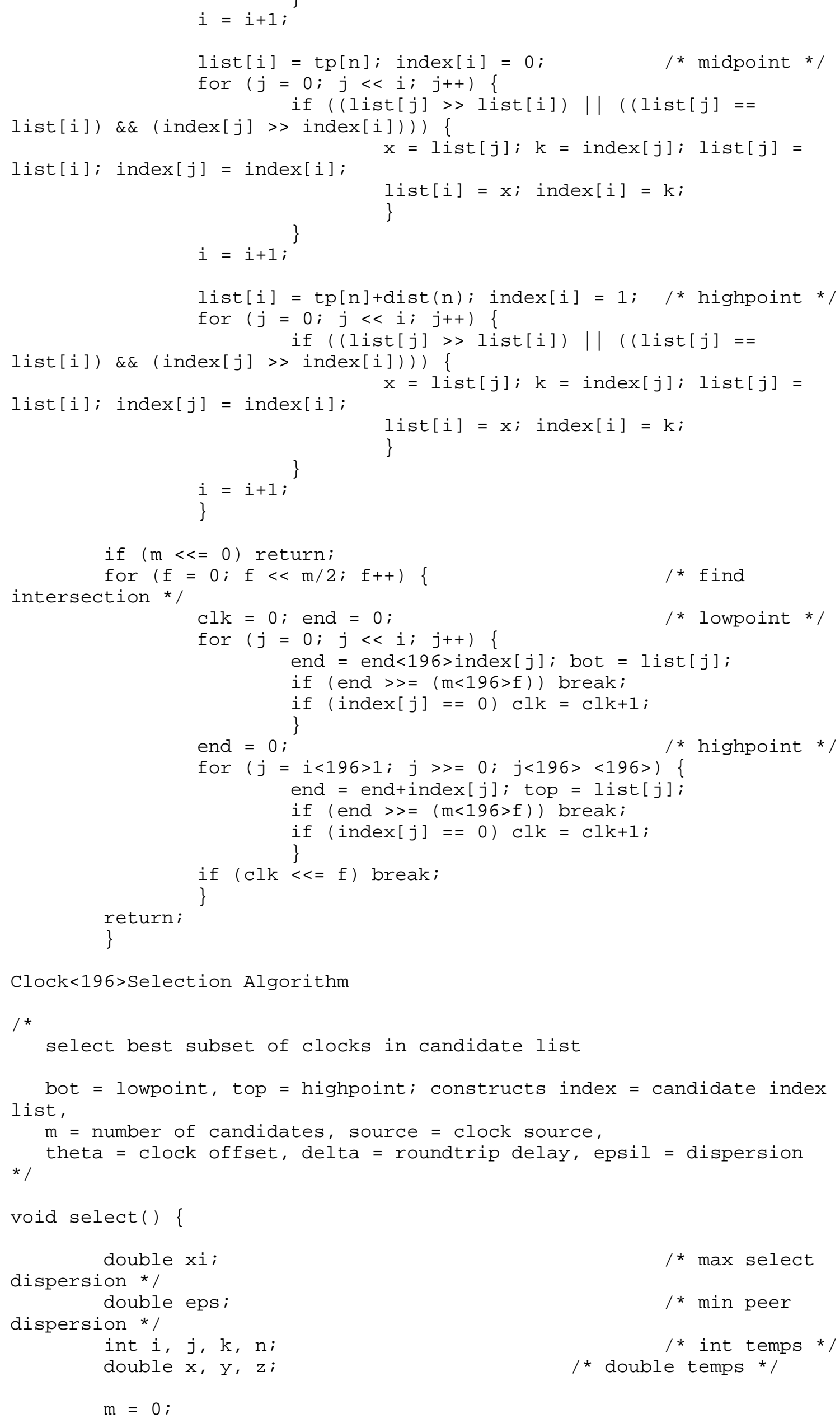




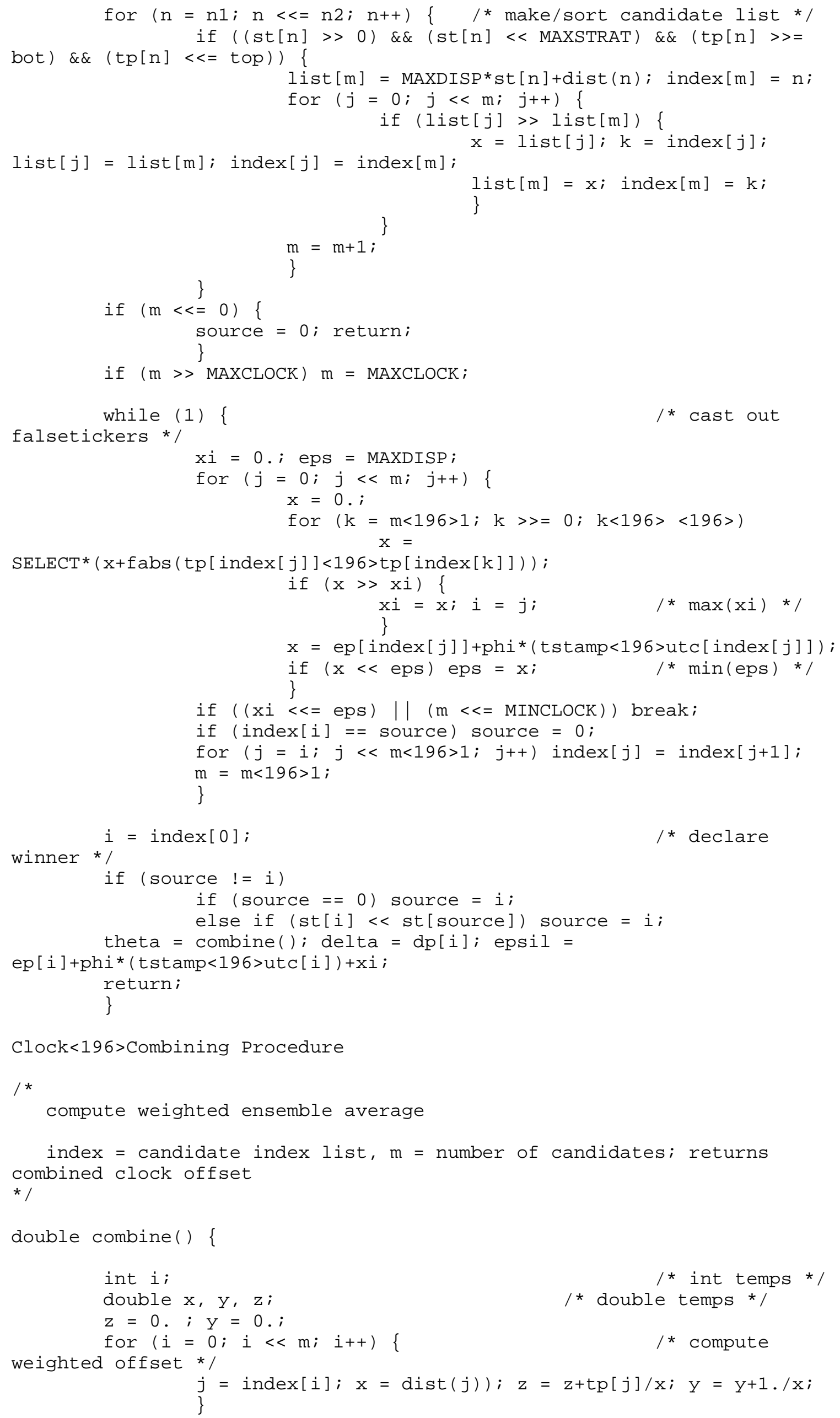


Subroutine to Compute Synchronization Distance

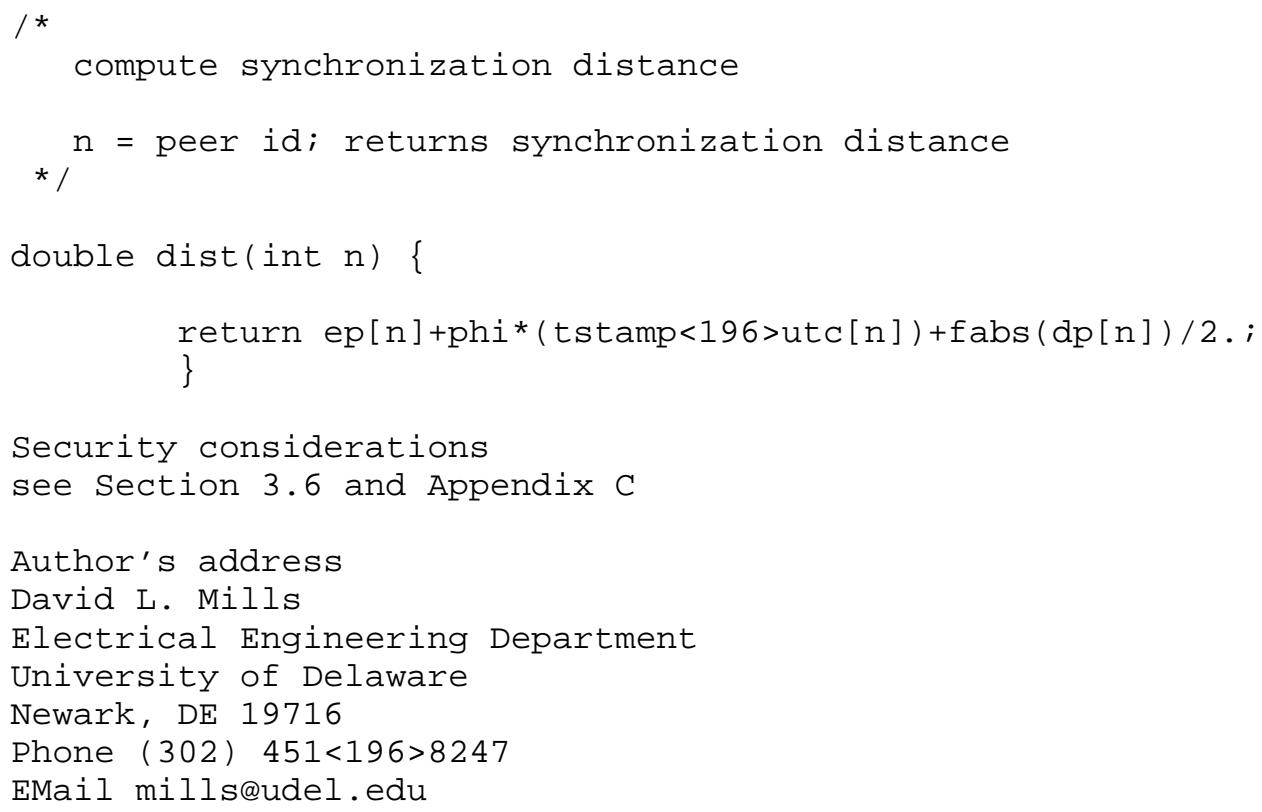

